\title{
The multi-dimensional Hermite-discontinuous Galerkin method for the Vlasov-Maxwell equations
}

\author{
O. Koshkarov, ${ }^{\mathrm{a}}$ G. Manzini, ${ }^{\mathrm{a}}$ G. L. Delzanno, ${ }^{\mathrm{a}}$ C. Pagliantini, ${ }^{\mathrm{b}}$ V. Roytershteyn ${ }^{\mathrm{c}}$ \\ ${ }^{a}$ T-5 Applied Mathematics and Plasma Physics Group, Los Alamos National Laboratory, Los Alamos, NM 87545, USA \\ b Department of Mathematics and Computer Science, Eindhoven University of Technology, Eindhoven, The Netherlands \\ ${ }^{\mathrm{c}}$ Space Science Institute, 4750 Walnut St, Suite 205, Boulder, CO 80301
}

\begin{abstract}
We discuss the development, analysis, implementation, and numerical assessment of a spectral method for the numerical simulation of the three-dimensional Vlasov-Maxwell equations. The method is based on a spectral expansion of the velocity space with the asymmetrically weighted Hermite functions. The resulting system of time-dependent nonlinear equations is discretized by the discontinuous Galerkin (DG) method in space and by the method of lines for the time integration using explicit Runge-Kutta integrators. The resulting code, called Spectral Plasma Solver (SPS-DG), is successfully applied to standard plasma physics benchmarks to demonstrate its accuracy, robustness, and parallel scalability.
\end{abstract}

Key words: 3-D Vlasov-Maxwell equations, AW Hermite discretization, Discontinuous Galerkin method

\section{Introduction}

Efficient and accurate coupling of the microscopic physics into the macroscopic system-scale dynamics (also known as fluid-kinetic coupling) is arguably the most important and yet still unresolved problem of computational plasma physics. It impacts a wide variety of systems, including the solar corona, the Earth's magnetosphere, all the way to laboratory experiments such as those for magnetic and inertial fusion energy. The crux of the matter is the large scale separation involved in plasma dynamics. For instance, for the Earth's magnetosphere, the local plasma Debye length (an important parameter governing the microscopic physics) can easily be less than $\sim 1 \mathrm{~m}$, while the system scale is $\sim 10^{9} \mathrm{~m}$. In what follows we will use the terms microscopic or kinetic and macroscopic or fluid interchangeably.

Recognizing the inability to solve this enormous scale separation at the microscopic level with available (and foreseeable) supercomputers, the next step is to develop methods that treat fluid-kinetic coupling in some approximated form and this is under active development. In one approach, the microscopic physics is treated only locally in selected regions of physical space by embedding a kinetic solver within a largescale fluid framework [24, 37, 52, 58, 81, 83]. Another approach seeks "augmented" fluid models, with better closures of the fluid equations that improve the representation of the kinetic physics [87, 88]. A different line of investigations targets large-scale simulations by averaging out certain scales of the system, as in the gyrokinetics approach [17] for magnetic fusion energy applications, or by treating electrons as a fluid (hence removing electron kinetic scales) while the ions are still treated kinetically, as in the hybrid approach [56, 62, 63, 71, 90]. 
An alternative method for fluid-kinetic coupling is based on a spectral expansion of the plasma distribution function at the kinetic level. With a suitable choice of the spectral basis, fluid-kinetic coupling is an intrinsic property of spectral methods: the low-order coefficients of the expansion are akin to a fluid description of the plasma, while the kinetic physics is captured by retaining additional terms in the expansion [21, 84]. (A proof-of-principle demonstration of how fluid-kinetic coupling can be exploited in spectral methods can be found in Ref. [84].) One can therefore recognize that spectral methods might offer the optimal way to treat microscopic physics in large-scale simulations since one can envision adapting the spectral expansion in time and in space to minimize/optimize the number of degrees of freedom for a given accuracy. It is also important to notice that spectral methods enclose the two approaches for fluid-kinetic coupling described in the previous paragraph: they can be seen as improved fluid models that treat kinetic physics in a reduced way but also as methods where the kinetic physics can be treated only locally, where necessary. It is also worth emphasizing that spectral methods for the solution of the kinetic equations are important in their own right, beyond fluid-kinetic coupling. These methods date back to the seventies $[2,45,57]$, where their application was limited to one-dimensional electrostatic problems, and their development continues to this day $[18,19,20,38,40,41,42,53,64,67,68,73,74,78,84]$. Currently, some drawbacks of spectral methods for the kinetic equations are their inability to enforce the positivity of the distribution function and, for some expansions (e.g. the asymmetrically-weighted (AW) Hermite representation), the lack of a numerical stability theorem. The optimization of the expansion basis is also an important open problem. In addition, we also note that on simple one-dimensional electrostatic problems spectral methods based on the AWHermite presentation were shown to be orders of magnitude faster/more accurate than Particle-In-Cell (PIC) methods [20]. References [39, 76] also present some physics applications of the AW-Hermite spectral method that were too computationally expensive for PIC.

To the best of our knowledge, the only implementation of a spectral method that treats the full threedimensional Vlasov-Maxwell equations is discussed in Refs. [38, 77, 85] and led to the development of the Spectral Plasma Solver (SPS) code [77, 85]. In those works, the physical space is discretized with a Fourier expansion, while the velocity space is discretized with an (asymmetrically weighted) Hermite expansion. The resulting numerical method features the conservation of total mass, momentum, and energy in a finite time step [38]. This approach is highly accurate and is particularly well suited for problems involving periodic boundary conditions and wave-like perturbations. For example, it has been successfully applied to studies of the turbulent cascade in magnetized plasmas $[76,77]$. However, a Fourier decomposition in physical space leads to multiple convolutions in the transformed equations, which result from nonlinear terms in the original equations. From a practical point of view, convolutions are handled using the pseudo-spectral method, which requires computing many Fast Fourier Transforms (FFTs) at every time step. In a parallel code, the FFTs involve global communication operations, which limit the code scalability and overall performance. To overcome this problem, in this paper we present a spectral method based on a finite element, discontinuousGalerkin (DG) discretization in physical space, coupled with a Hermite representation of velocity space.

The DG method was initially introduced for solving the neutron transport equation $[60,75]$ and was later extended to the numerical approximation of nonlinear conservation laws and hyperbolic system of partial differential equations [34, 35, 36]. We refer the reader to [32] for an historical overview and to [51, 79] for a general presentation. Relevant to our work are the papers for the Vlasov-Poisson system [3, 4, 5], the Boltzmann-Poisson system [10, 28, 29, 30, 31, 69], the Maxwell equations [33], and the Vlasov-Maxwell system $[26,27,52,55]$. Applications of the DG algorithm to reduced plasma models also exist, e.g. [65, $66,80]$. Unlike previous approaches, in this work we couple the DG discretization of the spatial terms of the Vlasov and Maxwell equations with a spectral representation of the velocity space. The formulation of our method is obtained by testing the conservative form of the partial differential equations against elements of a finite dimensional space of globally discontinuous functions, whose restriction to any element of the computational mesh is a polynomial of a maximum assigned degree. Therefore, the approximation has an intrinsic local conservative nature. Moreover, the accuracy of the method is determined by the degree of the local polynomials, so that increasing arbitrarily such parameter makes it possible to obtain numerical approximations of arbitrary order of accuracy. Consistent upwind numerical flux functions provide the exchange of information between adjacent cells so that the DG method is characterized by an extreme locality in data and communication. Importantly, this yields a method which is much better suited for 
implementations on high performance parallel architectures than the equivalent method based on a Fourier discretization in physical space which involves FFTs and global communications. Upwind numerical fluxes are chosen from numerical stability considerations. Importantly, the elemental polynomial basis can be built independently in each mesh element, so different approximation degrees can be used in different elements as in the $h p$ refinement strategy, and (in the modal setting) almost independently of the geometric shape of the element, thus providing a significant mesh flexibility in the application. Indeed, the DG method, which was originally developed using discontinuous polynomials on triangles or quadrilaterals, can be extended to more general unstructured meshes with polygonal (2D) and polytopal (3D) cells [22]. Finally, even though this topic is not pursued in the present work, it is worth mentioning that it would be possible to incorporate a shock-capturing capability in the DG method in a very natural and straightforward way by using limiters in the calculation of numerical fluxes.

The outline of the paper is as follows. In Section 2, we introduce the mathematical model considered in this paper that describes the transport phenomena of different charged particle species in a collisionless plasma under the action of the self-consistent electromagnetic field. The behavior of each particle species is modeled through its distribution function that satisfies a time-dependent Vlasov equation in the six-dimensional phase space, i.e. three dimensions in space and velocity, respectively. The self-consistent electromagnetic field generated by the charged plasma particles satisfies Maxwell's equations. In Section 3, we apply the spectral and DG discretizations to the Vlasov equations. The expansion in Hermite basis functions removes the velocity dependence by transforming the Vlasov equations of each particle species in a nonlinear hyperbolic system of partial differential equations for the coefficients of the expansion that are still dependent on time and space. Then, this system of coefficients is discretized in space by applying the DG method. In Section 4, we apply the DG method to the discretization of the Maxwell equations reformulated in divergence form. Here, we introduce the central and upwind numerical flux as possible alternatives in the scheme. In Section 5, we present the semi-discrete conservation properties of the method. In Section 6 , the final system of time-dependent ordinary differential equations for the various expansion coefficients is advanced in time by applying a standard Runge-Kutta (RK) method. In Section 7, we discuss some important aspects of the implementation that are crucial to obtain a computationally efficient solver. We also investigate the parallel scalability of the current implementation, and show that the algorithm is scalable on a particular high-performance-computing platform. In Section 8, we assess the performance of the method in terms of accuracy and prove its reliability and robustness on a set of benchmark problems that are representative of plasma physics modeling situations. In Section 9, we present our final remarks and conclusions.

Notation and Normalization. We normalize the model equations as follows. Time $t$ is normalized to the electron plasma frequency $\omega_{p e}=\sqrt{e^{2} n_{0}^{e} / \varepsilon_{0} m^{e}}$, where $e$ is the elementary charge, $m^{e}$ is the electron mass, $\varepsilon_{0}$ is the permittivity of vacuum, and $n_{0}^{e}$ is a reference electron density. The velocity coordinate $v$ is normalized to the speed of light $c$; the spatial coordinate $x$ is normalized to the electron inertial length $d_{e}=c / \omega_{p e}$; the magnetic field $\boldsymbol{B}$ is normalized to a reference magnetic field $B_{0}$, and the electric field is normalized to $c B_{0}$. We denote the quantities regarding a given plasma species by the superscript $s$, which may take the specific values $s=e$ (electrons) and $s=i$ (ions). Accordingly, we denote the mass of the particles of species $s$ by $m^{s}$ and their charge by $q^{s}$. We normalize charge $q^{s}$ and mass $m^{s}$ to elementary charge $e$ and mass $m^{e}$, respectively. Finally, we define the cyclotron frequency of species $s$ as $\omega_{c s}=e B_{0} / m^{s}$.

\section{Vlasov-Maxwell equations}

The behavior of the particles of species $s$ in a collisionless magnetized plasma is described at any time instant $t>0$ by the nonnegative distribution function $f^{s}(\boldsymbol{x}, \boldsymbol{v}, t)$, where $\boldsymbol{x}$ denotes the position in the physical space $\Omega_{x}$ and $\boldsymbol{v}$ the position in the velocity space $\Omega_{v}$. Under the action of the self-consistent electric and magnetic fields $\boldsymbol{E}(\boldsymbol{x}, t)$ and $\boldsymbol{B}(\boldsymbol{x}, t)$ generated by the particles' motion, the distribution function of species $s$ satisfies the (normalized) Vlasov equation: 


$$
\frac{\partial f^{s}}{\partial t}+\boldsymbol{v} \cdot \nabla_{\boldsymbol{x}} f^{s}+\frac{q^{s}}{m^{s}} \frac{\omega_{c e}}{\omega_{p e}}(\boldsymbol{E}+\boldsymbol{v} \times \boldsymbol{B}) \cdot \nabla_{\boldsymbol{v}} f^{s}=0 .
$$

The electric and magnetic fields $\boldsymbol{E}=\left(E_{x}, E_{y}, E_{z}\right)^{T}$ and $\boldsymbol{B}=\left(B_{x}, B_{y}, B_{z}\right)^{T}$, with $T$ denoting the transpose, satisfy the time-dependent wave propagation equations

$$
\begin{aligned}
& \frac{\partial \boldsymbol{E}}{\partial t}-\nabla_{\boldsymbol{x}} \times \boldsymbol{B}=-\frac{\omega_{p e}}{\omega_{c e}} \boldsymbol{J}, \\
& \frac{\partial \boldsymbol{B}}{\partial t}+\nabla_{\boldsymbol{x}} \times \boldsymbol{E}=0,
\end{aligned}
$$

and the divergence equations

$$
\begin{aligned}
& \nabla_{\boldsymbol{x}} \cdot \boldsymbol{E}=\frac{\omega_{p e}}{\omega_{c e}} \rho, \\
& \nabla_{\boldsymbol{x}} \cdot \boldsymbol{B}=0 .
\end{aligned}
$$

In Equations (2) and (4), $\boldsymbol{J}$ and $\rho$ are the self-consistent electric current and charge density, respectively,

$$
\begin{gathered}
\rho(\boldsymbol{x}, t)=\sum_{s} q^{s} \int_{\Omega_{v}} f^{s}(\boldsymbol{x}, \boldsymbol{v}, t) d \boldsymbol{v}, \\
\boldsymbol{J}(\boldsymbol{x}, t)=\sum_{s} q^{s} \int_{\Omega_{v}} \boldsymbol{v} f^{s}(\boldsymbol{x}, \boldsymbol{v}, t) d \boldsymbol{v},
\end{gathered}
$$

where the summation is over all the plasma species denoted by $s$. We consider the unbounded velocity space $\Omega_{v}=\mathbb{R}^{3}$ and we assume that each distribution function $f^{s}$ is rapidly decaying for $|\boldsymbol{v}| \rightarrow \infty$, i.e., it decays proportionally to $\exp \left(-|\boldsymbol{v}|^{2}\right)$ [47]. This assumption is physically consistent with the Maxwellian velocity distribution of a plasma in thermodynamic equilibrium [48]. Similarly, we consider the closed bounded subset $\Omega_{x} \subset \mathbb{R}^{3}$ with boundary $\Gamma_{x}$, and we assume that suitable problem-dependent boundary conditions for $f^{s}, \boldsymbol{E}$, and $\boldsymbol{B}$ are provided at $\Gamma_{x}$ for any time $t$ and any value of $\boldsymbol{v}$ in $\Omega_{v}$. Moreover, physically meaningful initial conditions must be provided for the unknown fields $f^{s}, \boldsymbol{E}, \boldsymbol{B}$ at the initial time $t=0$. Finally, when periodic boundary conditions are used,

$$
\int_{\Omega_{x}} \rho(\boldsymbol{x}, t) d \boldsymbol{x}=0 \quad t \geq 0
$$

and

$$
\int_{\Omega_{x}} \boldsymbol{J}(\boldsymbol{x}, t) d \boldsymbol{x}=0 \quad t \geq 0
$$

must be satisfied so that the charge density $\rho$ satisfies the global charge neutrality condition and the total current is zero.

\section{Hermite-DG discretization of the Vlasov equation}

\subsection{Spectral discretization in velocity space using Hermite functions}

We expand the distribution function $f^{s}$ on the multidimensional Hermite dual basis functions

$$
\Psi_{n, m, p}\left(\boldsymbol{\xi}^{s}\right)=\psi_{n}\left(\xi_{x}^{s}\right) \psi_{m}\left(\xi_{y}^{s}\right) \psi_{p}\left(\xi_{z}^{s}\right) \quad \text { and } \quad \Psi^{n, m, p}\left(\boldsymbol{\xi}^{s}\right)=\psi^{n}\left(\xi_{x}^{s}\right) \psi^{m}\left(\xi_{y}^{s}\right) \psi^{p}\left(\xi_{z}^{s}\right),
$$

for $n=0, \ldots, N_{v_{x}}, m=0, \ldots, N_{v_{y}}, p=0, \ldots, N_{v_{z}}$, where

$$
\boldsymbol{\xi}^{s}=\left(\xi_{x}^{s}, \xi_{y}^{s}, \xi_{z}^{s}\right)^{T}, \quad \xi_{x}^{s}=\frac{v_{x}-u_{x}^{s}}{\alpha_{x}^{s}}, \quad \xi_{y}^{s}=\frac{v_{y}-u_{y}^{s}}{\alpha_{y}^{s}}, \quad \xi_{z}^{s}=\frac{v_{z}-u_{z}^{s}}{\alpha_{z}^{s}} .
$$

In this paper, the quantities $u_{x}^{s}, u_{y}^{s}, u_{z}^{s}$ and $\alpha_{x}^{s}, \alpha_{y}^{s}, \alpha_{z}^{s}$ are constant factors that depend on the plasma species and that are provided by the user for a specific problem. (Note that, in general, it can be beneficial to 
allow these quantities to vary in both space and time, see for instance Refs. [40, 86], but this is left for future work). The Hermite functions $\Psi_{n, m, p}$ and $\Psi^{n, m, p}$ are given by the tensor product of the univariate asymmetrically weighted Hermite functions

$$
\begin{aligned}
\psi_{\zeta}\left(\xi_{\beta}^{s}\right) & =\left(\pi 2^{\zeta} \zeta !\right)^{-\frac{1}{2}} \mathcal{H}_{\zeta}\left(\xi_{\beta}^{s}\right) \exp \left(-\left(\xi_{\beta}^{s}\right)^{2}\right), \\
\psi^{\zeta}\left(\xi_{\beta}^{s}\right) & =\left(2^{\zeta} \zeta !\right)^{-\frac{1}{2}} \mathcal{H}_{\zeta}\left(\xi_{\beta}^{s}\right),
\end{aligned}
$$

where $\mathcal{H}_{\zeta}\left(\xi_{\beta}^{s}\right)$ is the $\zeta$-th univariate Hermite polynomial for $\zeta \in\{n, m, p\}$ and $\beta(\zeta) \in\{x, y, z\}$. The orthogonality of the Hermite polynomials $\mathcal{H}_{\zeta}\left(\xi_{\beta}^{s}\right)$ and $\mathcal{H}_{\zeta^{\prime}}\left(\xi_{\beta}^{s}\right)$ with respect to the weighted $L^{2}$-inner product with weight $\exp \left(-\left(\xi_{\beta}^{s}\right)^{2}\right)$ induces the duality relation between $\Psi_{n, m, p}$ and $\Psi^{n^{\prime}, m^{\prime}, p^{\prime}}$

$$
\left\langle\Psi_{n, m, p}, \Psi^{n^{\prime}, m^{\prime}, p^{\prime}}\right\rangle=\int_{\Omega_{v}} \Psi_{n, m, p}\left(\boldsymbol{\xi}^{s}\right) \Psi^{n^{\prime}, m^{\prime}, p^{\prime}}\left(\boldsymbol{\xi}^{s}\right) d \boldsymbol{\xi}^{s}=\delta_{n, n^{\prime}} \delta_{m, m^{\prime}} \delta_{p, p^{\prime}}
$$

The normalization factors in (9)-(10) are chosen to insure orthonormality in (11). The recursive property and the derivative formula of the Hermite polynomials imply the following relations for the Hermite functions:

$$
\begin{aligned}
v_{\beta} \psi_{\zeta}\left(\xi_{\beta}^{s}\right) & =\alpha_{\beta}^{s} \sqrt{\frac{\zeta+1}{2}} \psi_{\zeta+1}\left(\xi_{\beta}^{s}\right)+\alpha_{\beta}^{s} \sqrt{\frac{\zeta}{2}} \psi_{\zeta-1}\left(\xi_{\beta}^{s}\right)+u_{\beta}^{s} \psi_{\zeta}\left(\xi_{\beta}^{s}\right), \\
\frac{d \psi_{\zeta}}{d v_{\beta}}\left(\xi_{\beta}^{s}\right) & =-\frac{\sqrt{2(\zeta+1)}}{\alpha_{\beta}^{s}} \psi_{\zeta+1}\left(\xi_{\beta}^{s}\right) .
\end{aligned}
$$

The numerical approximation at $t \geq 0$ of the distribution function $f^{s}$ is given by the finite expansion

$$
f^{s}(\boldsymbol{x}, \boldsymbol{v}, t) \approx f^{s, H}(\boldsymbol{x}, \boldsymbol{v}, t)=\sum_{n=0}^{N_{v_{x}}} \sum_{m=0}^{N_{v_{y}}} \sum_{p=0}^{N_{v_{z}}} C_{n, m, p}^{s}(\boldsymbol{x}, t) \psi_{n}\left(\xi_{x}^{s}\right) \psi_{m}\left(\xi_{y}^{s}\right) \psi_{p}\left(\xi_{z}^{s}\right),
$$

where the summation on $n, m$, and $p$ is truncated at $N_{v_{x}}, N_{v_{y}}$, and $N_{v_{z}}$, respectively. The expansion coefficients $C_{n, m, p}^{s}(\boldsymbol{x}, 0)$ at the initial time $t=0$ are

$$
C_{n, m, p}^{s}(\boldsymbol{x}, 0)=\int_{\Omega_{v}} f^{s}(\boldsymbol{x}, \boldsymbol{v}, 0) \Psi^{n, m, p}\left(\boldsymbol{\xi}^{s}\right) d \boldsymbol{\xi}^{s} .
$$

To derive the time-dependent nonlinear system for the Hermite expansion coefficients $C^{s}=\left\{C_{n, m, p}^{s}\right\}$, we multiply (1) by $\Psi^{n, m, p}\left(\boldsymbol{\xi}^{s}\right)$ and integrate over $\Omega_{v}$,

$$
\int_{\Omega_{v}}\left(\frac{\partial f^{s, H}}{\partial t}+\boldsymbol{v} \cdot \nabla_{\boldsymbol{x}} f^{s, H}+\frac{q^{s}}{m^{s}} \frac{\omega_{c e}}{\omega_{p e}}(\boldsymbol{E}+\boldsymbol{v} \times \boldsymbol{B}) \cdot \nabla_{\boldsymbol{v}} f^{s, H}\right) \Psi^{n, m, p}\left(\boldsymbol{\xi}^{s}\right) d \boldsymbol{\xi}^{s}=0
$$

Then, we substitute the finite expansion (14) of $f^{s, H}$, integrate by parts the derivative term in $\boldsymbol{v}$, and obtain:

$$
\frac{\partial C_{n, m, p}^{s}(\boldsymbol{x}, t)}{\partial t}+\mathcal{L}_{n, m, p}\left(C^{s}\right)+\mathcal{N}_{n, m, p}\left(C^{s}\right)=0,
$$

where the "linear" term $\mathcal{L}_{n, m, p}$ and the "nonlinear" term $\mathcal{N}_{n, m, p}$ are given by

$$
\mathcal{L}_{n, m, p}\left(C^{s}\right)=\int_{\Omega_{v}} \boldsymbol{v} \cdot \nabla_{\boldsymbol{x}} f^{s, H}(\boldsymbol{x}, \boldsymbol{v}, t) \Psi^{n, m, p}\left(\boldsymbol{\xi}^{s}\right) d \boldsymbol{\xi}^{s},
$$

and

$$
\mathcal{N}_{n, m, p}\left(C^{s}\right)=\frac{q^{s}}{m^{s}} \frac{\omega_{c e}}{\omega_{p e}} \int_{\Omega_{v}}\left((\boldsymbol{E}(\boldsymbol{x}, t)+\boldsymbol{v} \times \boldsymbol{B}(\boldsymbol{x}, t)) \cdot \nabla_{\boldsymbol{v}} f^{s, H}(\boldsymbol{x}, \boldsymbol{v}, t)\right) \Psi^{n, m, p}\left(\boldsymbol{\xi}^{s}\right) d \boldsymbol{\xi}^{s} .
$$

A straightforward calculation using formulas (9) and (10), together with the orthogonality property (11) of the Hermite basis functions yields the set of evolution equations for the Hermite coefficient $C_{n, m, p}^{s}(\boldsymbol{x}, t)$, for any triplet $(n, m, p)$, which reads 


$$
\begin{aligned}
& \frac{\partial C_{n, m, p}^{s}}{\partial t}+\alpha_{x}^{s}\left(\sqrt{\frac{n+1}{2}} \frac{\partial C_{n+1, m, p}^{s}}{\partial x}+\sqrt{\frac{n}{2}} \frac{\partial C_{n-1, m, p}^{s}}{\partial x}+\frac{u_{x}^{s}}{\alpha_{x}^{s}} \frac{\partial C_{n, m, p}^{s}}{\partial x}\right) \\
& +\alpha_{y}^{s}\left(\sqrt{\frac{m+1}{2}} \frac{\partial C_{n, m+1, p}^{s}}{\partial y}+\sqrt{\frac{m}{2}} \frac{\partial C_{n, m-1, p}^{s}}{\partial y}+\frac{u_{y}^{s}}{\alpha_{y}^{s}} \frac{\partial C_{n, m, p}^{s}}{\partial y}\right) \\
& +\alpha_{z}^{s}\left(\sqrt{\frac{p+1}{2}} \frac{\partial C_{n, m, p+1}^{s}}{\partial z}+\sqrt{\frac{p}{2}} \frac{\partial C_{n, m, p-1}^{s}}{\partial z}+\frac{u_{z}^{s}}{\alpha_{z}^{s}} \frac{\partial C_{n, m, p}^{s}}{\partial z}\right) \\
& -\frac{q^{s}}{m^{s}} \frac{\omega_{c e}}{\omega_{p e}}\left(\frac{\sqrt{2 n}}{\alpha_{x}^{s}} E_{x} C_{n-1, m, p}^{s}+\frac{\sqrt{2 m}}{\alpha_{y}^{s}} E_{y} C_{n, m-1, p}^{s}+\frac{\sqrt{2 p}}{\alpha_{z}^{s}} E_{z} C_{n, m, p-1}^{s}\right) \\
& -\frac{q^{s}}{m^{s}} \frac{\omega_{c e}}{\omega_{p e}} B_{x}\left[\sqrt{m p}\left(\frac{\alpha_{z}^{s}}{\alpha_{y}^{s}}-\frac{\alpha_{y}^{s}}{\alpha_{z}^{s}}\right) C_{n, m-1, p-1}^{s}+\sqrt{m(p+1)} \frac{\alpha_{z}^{s}}{\alpha_{y}^{s}} C_{n, m-1, p+1}^{s}\right. \\
& \left.-\sqrt{(m+1) p} \frac{\alpha_{y}^{s}}{\alpha_{z}^{s}} C_{n, m+1, p-1}^{s}+\sqrt{2 m} \frac{u_{z}^{s}}{\alpha_{y}^{s}} C_{n, m-1, p}^{s}-\sqrt{2 p} \frac{u_{y}^{s}}{\alpha_{z}^{s}} C_{n, m, p-1}^{s}\right] \\
& -\frac{q^{s}}{m^{s}} \frac{\omega_{c e}}{\omega_{p e}} B_{y}\left[\sqrt{p n}\left(\frac{\alpha_{x}^{s}}{\alpha_{z}^{s}}-\frac{\alpha_{z}^{s}}{\alpha_{x}^{s}}\right) C_{n-1, m, p-1}^{s}+\sqrt{p(n+1)} \frac{\alpha_{x}^{s}}{\alpha_{z}^{s}} C_{n+1, m, p-1}^{s}\right. \\
& \left.-\sqrt{(p+1) n} \frac{\alpha_{z}^{s}}{\alpha_{x}^{s}} C_{n-1, m, p+1}^{s}+\sqrt{2 p} \frac{u_{x}^{s}}{\alpha_{z}^{s}} C_{n, m, p-1}^{s}-\sqrt{2 n} \frac{u_{z}^{s}}{\alpha_{x}^{s}} C_{n-1, m, p}^{s}\right] \\
& -\frac{q^{s}}{m^{s}} \frac{\omega_{c e}}{\omega_{p e}} B_{z}\left[\sqrt{n m}\left(\frac{\alpha_{y}^{s}}{\alpha_{x}^{s}}-\frac{\alpha_{x}^{s}}{\alpha_{y}^{s}}\right) C_{n-1, m-1, p}^{s}+\sqrt{n(m+1)} \frac{\alpha_{y}^{s}}{\alpha_{x}^{s}} C_{n-1, m+1, p}^{s}\right. \\
& \left.-\sqrt{(n+1) m} \frac{\alpha_{x}^{s}}{\alpha_{y}^{s}} C_{n+1, m-1, p}^{s}+\sqrt{2 n} \frac{u_{y}^{s}}{\alpha_{x}^{s}} C_{n-1, m, p}^{s}-\sqrt{2 m} \frac{u_{x}^{s}}{\alpha_{y}^{s}} C_{n, m-1, p}^{s}\right]=0 .
\end{aligned}
$$

By comparison with (17), (18), (19), one can observe that the first three terms in the parenthesis after the time derivative, which contain the spatial derivatives of the Hermite coefficients, derive from $\mathcal{L}\left(C^{s}\right)$ and the subsequent ones ensue from $\mathcal{N}\left(C^{s}\right)$. Details about the derivation of Eq. (20) are reported in the final appendix for completeness.

\subsection{Discontinuous Galerkin approximation in configuration space}

We adopt the usual notation from finite difference schemes on Cartesian grids. We partition the space domain $\Omega_{x}$ into $N_{c}=N_{x} N_{y} N_{z}$ cubic or regular hexahedral cells, so that we have $N_{x}$ mesh elements in the $x$-direction, $N_{y}$ elements in the $y$-direction, and $N_{z}$ elements in the $z$-direction. These partitions are labeled by the latin indices $i, j, k$ running from 1 to $N_{x}, N_{y}$, and $N_{z}$, respectively. For convenience of exposition, we label the generic mesh cell by the letter $I$ and express the summation over all mesh cells by $\sum_{I}$ (without specifying the summation bounds). With some abuse of notation, we may subindex $I$ as $I_{i, j, k}$, so that, for example, two consecutive cells in the $x$-direction are denoted by $I_{i, j, k}$ and $I_{i+1, j, k}$ and are separated by the cell interface $\mathrm{f}_{i+\frac{1}{2}, j, k}$. We denote the position of the cell center $I_{i, j, k}$ by $\boldsymbol{x}_{i, j, k}=\left(x_{i, j, k}, y_{i, j, k}, z_{i, j, k}\right)$, and the size of such cell along the $x^{-}, y$-, and $z$-directions by $\Delta x_{i, j, k}, \Delta y_{i, j, k}$, and $\Delta z_{i, j, k}$, so that

$$
\begin{aligned}
I \equiv I_{i, j, k}= & {\left[-\frac{\Delta x_{i, j, k}}{2}+x_{i, j, k}, \frac{\Delta x_{i, j, k}}{2}+x_{i, j, k}\right] \times\left[-\frac{\Delta y_{i, j, k}}{2}+y_{i, j, k}, \frac{\Delta y_{i, j, k}}{2}+y_{i, j, k}\right] \times } \\
& {\left[-\frac{\Delta z_{i, j, k}}{2}+z_{i, j, k}, \frac{\Delta z_{i, j, k}}{2}+z_{i, j, k}\right] . }
\end{aligned}
$$


For the exposition's sake, we may assume that the cells are all equispaced, and consider $\Delta x, \Delta y$, and $\Delta z$ as the mesh size steps in the three spatial directions. Accordingly, triplets with an half-integer index, e.g. $\left(i \pm \frac{1}{2}, j, k\right),\left(i, j \pm \frac{1}{2}, k\right)$ and $\left(i, j, k \pm \frac{1}{2}\right)$, denote the cell interfaces that are orthogonal to the $x$-, $y$-, and $z$-direction, respectively, and delimiting cell $I_{i, j, k}$. The faces are oriented such that the normal vector to each face always points outwards.

Next, we consider the space of polynomials of degree up to $N_{D G}$ defined on $I$, which we denote by $\mathbb{P}_{N_{D G}}(I)$. We do not assume any continuity or weaker regularity condition at the interface shared by two consecutive cells. Therefore, any function defined on $\Omega_{x}$ whose restriction to any mesh cell is the product of univariate polynomials of degree (at most) $N_{D G}$, may be discontinuous at any cell interface. We denote the basis for the local polynomial space on cell $I$ by $\left\{\varphi^{I, l}\right\}$ for $l=1, \ldots, N_{l}$, where $N_{l}$ is the cardinality of $\mathbb{P}_{N_{D G}}(I)$, so that $\mathbb{P}_{N_{D G}}(I)=\operatorname{span}\left\{\varphi^{I, l}\right\}_{l}$. We recall that $N_{l}=N_{D G}+1$ for univariate polynomials, $N_{l}=\left(N_{D G}+1\right)\left(N_{D G}+2\right) /$ 2 for bivariate polynomials, and $N_{l}=\left(N_{D G}+1\right)\left(N_{D G}+2\right)\left(N_{D G}+3\right) / 6$ for trivariate polynomials.

The discontinuous Galerkin approximation of the spectral coefficients $C_{n, m, p}^{s}(\boldsymbol{x}, t)$, for a fixed triple $(n, m, p)$ and in every cell $I$, is given by expanding the Hermite coefficient in the local polynomial basis of the space $\mathbb{P}_{N_{D G}}(I)$ :

$$
C_{n, m, p}^{s}(\boldsymbol{x}, t) \approx C_{n, m, p}^{s, D G}(\boldsymbol{x}, t):=\sum_{l=1}^{N_{l}} C_{n, m, p}^{s, I, l}(t) \varphi^{I, l}(\boldsymbol{x}), \quad \forall \boldsymbol{x} \in I .
$$

Collecting the local expansions, we obtain the (possibly discontinuous) global approximation of the spectral coefficients

$$
C_{n, m, p}^{s, D G}(\boldsymbol{x}, t)=\sum_{I} \sum_{l=1}^{N_{l}} C_{n, m, p}^{s, I, l}(t) \varphi^{I, l}(\boldsymbol{x}), \quad \forall \boldsymbol{x} \in \Omega_{x},
$$

and, accordingly, the Hermite-DG approximation of the distribution function $f^{s}$ is given by

$$
f^{s, N}(\boldsymbol{x}, \boldsymbol{v}, t):=\sum_{n, m, p} C_{n, m, p}^{s, D G}(\boldsymbol{x}, t) \Psi_{n, m, p}\left(\boldsymbol{\xi}^{s}\right)=\sum_{n, m, p} \sum_{I, l} C_{n, m, p}^{s, I, l}(t) \Psi_{n, m, p}\left(\boldsymbol{\xi}^{s}\right) \varphi^{I, l}(\boldsymbol{x}),
$$

where, to ease the notation, we did not specify the summation bounds.

Remark 3.1 To avoid using a cumbersome notation, hereafter, we will remove the superscript DG from the symbols denoting the approximate " $C$ " " coefficients. Therefore, we keep using $C_{n, m, p}^{s}$ instead of $C_{n, m, p}^{s, D}$, but intending that these quantities refer to the DG expansion with a finite number of spatial modes.

Remark 3.2 It is worth noting that $f^{s, H}$ and $f^{s, N}$ are different functions. Indeed, $f^{s, H}$ for each species $s$ is the solution of the variational problem (16) and is an approximation of the corresponding distribution function $f^{s}$ solving (1) after the truncation of the expansion on the Hermite velocity basis. Instead, $f^{s, N}$ is the discontinuous Galerkin approximation of $f^{s, H}$ and is the solution of the discrete variational problem that is constructed in the rest of this section. Since the numerical method is formulated in terms of the finite set of " $C$ " " coefficients, we do not need to know the specific discrete equation satisfied by $f^{s, N}$ for the implementation. Such equation is used in the analysis of the conservation properties of the semi-discrete method in Appendix B.

Let $L_{\zeta}$ denote the univariate Legendre polynomial of degree $\zeta$ in the interval $[-1,1]$. The set of polynomials $\left\{L_{\zeta}\right\}_{\zeta=0}^{N_{D G}}$ forms an orthogonal basis for $\mathbb{P}_{N_{D G}}([-1,1])[44]$. We construct the multidimensional functions $\varphi^{I, l}(\boldsymbol{x})$ as the tensor product of rescaled and translated univariate Legendre polynomials as follows,

$$
\varphi^{I, l}(\boldsymbol{x})=L_{l_{x}}\left(2 \frac{x-x_{i, j, k}}{\Delta x}\right) L_{l_{y}}\left(2 \frac{y-y_{i, j, k}}{\Delta y}\right) L_{l_{z}}\left(2 \frac{z-z_{i, j, k}}{\Delta z}\right),
$$

where $l_{x}, l_{y}, l_{z}=0, \ldots, N_{D G}$ and $l_{x}+l_{y}+l_{z} \leq N_{D G}$. The index $l$ is a convenient mapping onto integer numbers that enumerates the triplets $\left(l_{x}, l_{y}, l_{z}\right)$ from 1 to $N_{l}$. The orthogonality of the Legendre polynomials allows us to simplify the calculation in the discontinuous Galerkin formulation. Indeed, we perform the integration 
analitically whenever we can reduce the multidimensional integration involving the basis functions $\varphi^{I, l}$ to the exact one-dimensional integration formulas

$$
\begin{aligned}
\int_{-1}^{1} L_{i}(\sigma) L_{j}(\sigma) d \sigma & =\frac{2}{2 i+1} \delta_{i, j}, \\
\int_{-1}^{1} L_{i}(\sigma) \frac{d L_{j}(\sigma)}{d \sigma} d \sigma & = \begin{cases}2, & \text { if } i<j \text { and } j-i \text { is odd } \\
0, & \text { otherwise, }\end{cases} \\
\int_{-1}^{1} L_{i}(\sigma) L_{j}(\sigma) L_{k}(\sigma) d \sigma & =2\left(\begin{array}{lll}
i & j & k \\
0 & 0 & 0
\end{array}\right)^{2},
\end{aligned}
$$

where the special form of Wigner 3 -j symbol in the right-hand side of $(24 \mathrm{c})$ is defined as $[1,89]$ :

$$
\left(\begin{array}{lll}
i & j & k \\
0 & 0 & 0
\end{array}\right)= \begin{cases}(-1)^{\ell} \frac{\ell !}{(\ell-i) !(\ell-j) !(\ell-k) !} \sqrt{\frac{(2 \ell-2 i) !(2 \ell-2 j) !(2 \ell-2 k) !}{(2 \ell+1) !},} & \text { for } 2 \ell=i+j+k \text { even } \\
0, & \text { otherwise. }\end{cases}
$$

To derive the discontinuous Galerkin approximation of the Vlasov equations, we multiply (17) by the generic basis function $\varphi^{I, l}$ and integrate over the space domain $\Omega_{x}$

$$
\int_{I}\left(\frac{\partial C_{n, m, p}^{s}}{\partial t}+\mathcal{L}_{n, m, p}\left(C^{s}\right)+\mathcal{N}_{n, m, p}\left(C^{s}\right)\right) \varphi^{I, l}(\boldsymbol{x}) d \boldsymbol{x}=0 .
$$

Note that the integral above has been restricted to the mesh element $I$ since $\varphi^{I, l}$ is zero outside $I$. We now consider each term separately. In the first term a direct substitution of (21) together with the orthogonality of the Legendre functions yields

$$
\int_{I} \frac{\partial C_{n, m, p}^{s}(t)}{\partial t} \varphi^{I, l}(\boldsymbol{x}) d \boldsymbol{x}=\sum_{l^{\prime}=1}^{N_{l}} \frac{d C_{n, m, p}^{s, I, l^{\prime}}(t)}{d t} \int_{I} \varphi^{I, l^{\prime}}(\boldsymbol{x}) \varphi^{I, l}(\boldsymbol{x}) d \boldsymbol{x}=\mu_{I, l} \frac{d C_{n, m, p}^{s, I, l}(t)}{d t},
$$

where the multiplicative factor $\mu_{I, l}:=\int_{I}\left(\varphi^{I, l}\right)^{2} d \boldsymbol{x}$ is the $(l, l)$ entry of the DG mass matrix (the mass matrix is diagonal in this case in view of the orthogonality properties of the Legendre polynomials).

\subsection{Linear terms}

In order to reformulate (20) in a more compact form we introduce the vector-valued function $\mathbf{C}^{s}(\boldsymbol{x}, t) \in$ $\mathbb{R}^{N}$, with $N:=\left(N_{v_{x}}+1\right)\left(N_{v_{y}}+1\right)\left(N_{v_{z}}+1\right)$, that collects the Hermite spectral coefficients for all $(\boldsymbol{x}, t)$. For a given vector $\boldsymbol{V} \in \mathbb{R}^{N}$, we denote by $[\boldsymbol{V}]_{n, m, p}$ the entry corresponding to the $(n, m, p)$-th triple, so that, for example, $\left[\mathbf{C}^{s}\right]_{n, m, p}:=C_{n, m, p}^{s}$. With this notation, we reformulate the linear term in (18) as,

$$
\mathcal{L}\left(\mathbf{C}^{s}\right)=\left(\mathbb{A}_{x} \frac{\partial}{\partial x}+\mathbb{A}_{y} \frac{\partial}{\partial y}+\mathbb{A}_{z} \frac{\partial}{\partial z}\right) \mathbf{C}^{s}
$$

where $\left[\mathcal{L}\left(\mathbf{C}^{s}\right)\right]_{n, m, p}=\mathcal{L}_{n, m, p}\left(C^{s}\right)$, and the matrices $\mathbb{A}_{x}, \mathbf{A}_{y}, \mathbf{A}_{z} \in \mathbb{R}^{N \times N}$ are defined according to (20) as,

$$
\begin{aligned}
& {\left[\mathbb{A}_{x} \frac{\partial \mathbf{C}^{s}}{\partial x}\right]_{n, m, p}=\alpha_{x}^{s}\left(\sqrt{\frac{n+1}{2}} \frac{\partial C_{n+1, m, p}^{s}}{\partial x}+\sqrt{\frac{n}{2}} \frac{\partial C_{n-1, m, p}^{s}}{\partial x}+\frac{u_{x}^{s}}{\alpha_{x}^{s}} \frac{\partial C_{n, m, p}^{s}}{\partial x}\right)} \\
& {\left[\mathbb{A}_{y} \frac{\partial \mathbf{C}^{s}}{\partial y}\right]_{n, m, p}=\alpha_{y}^{s}\left(\sqrt{\frac{m+1}{2}} \frac{\partial C_{n, m+1, p}^{s}}{\partial y}+\sqrt{\frac{m}{2}} \frac{\partial C_{n, m-1, p}^{s}}{\partial y}+\frac{u_{y}^{s}}{\alpha_{y}^{s}} \frac{\partial C_{n, m, p}^{s}}{\partial y}\right),} \\
& {\left[\mathbb{A}_{z} \frac{\partial \mathbf{C}^{s}}{\partial z}\right]_{n, m, p}=\alpha_{z}^{s}\left(\sqrt{\frac{p+1}{2}} \frac{\partial C_{n, m, p+1}^{s}}{\partial z}+\sqrt{\frac{p}{2}} \frac{\partial C_{n, m, p-1}^{s}}{\partial z}+\frac{u_{z}^{s}}{\alpha_{z}^{s}} \frac{\partial C_{n, m, p}^{s}}{\partial z}\right) .}
\end{aligned}
$$


Since $\mathbb{A}_{x}, \mathbb{A}_{y}$, and $\mathbb{A}_{z}$ are constant-valued matrices, we can directly consider their action on the vector of unknowns $\mathbf{C}^{s}$ and write

$$
\mathcal{L}\left(\mathbf{C}^{s}\right)=\frac{\partial}{\partial x}\left(\mathbb{A}_{x} \mathbf{C}^{s}\right)+\frac{\partial}{\partial y}\left(\mathbb{A}_{y} \mathbf{C}^{s}\right)+\frac{\partial}{\partial z}\left(\mathbb{A}_{z} \mathbf{C}^{s}\right),
$$

with the component-wise definitions

$$
\begin{aligned}
& {\left[\mathbb{A}_{x} \mathbf{C}^{s}\right]_{n, m, p}=\alpha_{x}^{s}\left(\sqrt{\frac{n+1}{2}} C_{n+1, m, p}^{s}+\sqrt{\frac{n}{2}} C_{n-1, m, p}^{s}+\frac{u_{x}^{s}}{\alpha_{x}^{s}} C_{n, m, p}^{s}\right),} \\
& {\left[\mathbb{A}_{y} \mathbf{C}^{s}\right]_{n, m, p}=\alpha_{y}^{s}\left(\sqrt{\frac{m+1}{2}} C_{n, m+1, p}^{s}+\sqrt{\frac{m}{2}} C_{n, m-1, p}^{s}+\frac{u_{y}^{s}}{\alpha_{y}^{s}} C_{n, m, p}^{s}\right),} \\
& {\left[\mathbb{A}_{z} \mathbf{C}^{s}\right]_{n, m, p}=\alpha_{z}^{s}\left(\sqrt{\frac{p+1}{2}} C_{n, m, p+1}^{s}+\sqrt{\frac{p}{2}} C_{n, m, p-1}^{s}+\frac{u_{z}^{s}}{\alpha_{z}^{s}} C_{n, m, p}^{s}\right) .}
\end{aligned}
$$

Relations (31a)-(31c) are used in the next section to introduce the upwind flux discretization of the Vlasov equations in the discontinuous Galerkin framework.

We split the cell boundary as $\partial I=\partial I_{x} \cup \partial I_{y} \cup \partial I_{z}$, where $\partial I_{x}=\mathrm{f}_{i+\frac{1}{2}, j, k} \cup \mathrm{f}_{i-\frac{1}{2}, j, k}, \partial I_{y}=\mathrm{f}_{i, j+\frac{1}{2}, k} \cup \mathrm{f}_{i, j-\frac{1}{2}, k}$, and $\partial I_{z}=\mathrm{f}_{i, j, k+\frac{1}{2}} \cup \mathrm{f}_{i, j, k-\frac{1}{2}}$. Integrating by parts the linear term of (26) and using (30) yields

$$
\begin{aligned}
\int_{I} \mathcal{L}_{n, m, p}\left(C^{s}\right) \varphi^{I, l}(\boldsymbol{x}) d \boldsymbol{x}= & \int_{I}\left[\sum_{\beta \in\{x, y, z\}} \mathbb{A}_{\beta} \frac{\partial \mathbf{C}^{s}(\boldsymbol{x}, t)}{\partial \beta}\right]_{n, m, p} \varphi^{I, l}(\boldsymbol{x}) d \boldsymbol{x} \\
= & \int_{I}\left[\sum_{\beta \in\{x, y, z\}} \frac{\partial}{\partial \beta}\left(\mathbb{A}_{\beta} \mathbf{C}^{s}(\boldsymbol{x}, t)\right)\right]_{n, m, p} \varphi^{I, l}(\boldsymbol{x}) d \boldsymbol{x} \\
= & \sum_{\beta \in\{x, y, z\}} \int_{I} \frac{\partial}{\partial \beta}\left[\mathbb{A}_{\beta} \mathbf{C}^{s}(\boldsymbol{x}, t)\right]_{n, m, p} \varphi^{I, l}(\boldsymbol{x}) d \boldsymbol{x} \\
= & -\sum_{\beta \in\{x, y, z\}} \int_{I}\left[\mathbb{A}_{\beta} \mathbf{C}^{s}(\boldsymbol{x}, t)\right]_{n, m, p} \frac{\partial \varphi^{I, l}(\boldsymbol{x})}{\partial \beta} d \boldsymbol{x} \\
& +\sum_{\beta \in\{x, y, z\}} \delta_{\beta}\left[\left[\mathbb{A}_{\beta} \mathbf{C}^{s}(\boldsymbol{x}, t)\right]_{n, m, p} \varphi^{I, l}(\boldsymbol{x})\right]_{\partial I_{\beta}},
\end{aligned}
$$

where

$$
\delta_{\beta}\left[\left[\mathbb{A}_{\beta} \mathbf{C}^{s}(\boldsymbol{x}, t)\right]_{n, m, p} \varphi^{I, l}(\boldsymbol{x})\right]_{\partial I_{\beta}}:=\int_{\partial I_{\beta}}\left[n_{\beta} \mathbb{A}_{\beta} \mathbf{C}^{s}(\boldsymbol{x}, t)\right]_{n, m, p} \varphi^{I, l}(\boldsymbol{x}) d S,
$$

are the terms at $\partial I$, the boundary of cell $I$, originating from the integration by parts, and $n_{\beta}, \beta \in\{x, y, z\}$, is the $\beta$ component of $\boldsymbol{n}=\left(n_{x}, n_{y}, n_{z}\right)^{T}$, the unit vector orthogonal to $\partial I$. Therefore, the right-hand side of (32) contains two integral terms: a volume integration term on $I$ and a surface integration term on $\partial I$. The volume integral is the sum of three independent volume integrals associated with the derivatives of $\varphi^{I, l}$ in the three directions $x, y$, and $z$. To exploit the orthogonality properties of the Legendre polynomials (24a) and (24b), we substitute expansion (21) and use formulas (31a), (31b), and (31c). Let us introduce the matrices $\boldsymbol{\Phi}_{\beta} \in \mathbb{R}^{N_{l} \times N_{l}}$, for $\beta \in\{x, y, z\}$, defined as

$$
\left(\boldsymbol{\Phi}_{\beta}\right)_{l, l^{\prime}}=\int_{I} \frac{\partial \varphi^{I, l}(\boldsymbol{x})}{\partial \beta} \varphi^{I, l^{\prime}}(\boldsymbol{x}) d \boldsymbol{x} .
$$

Then, 


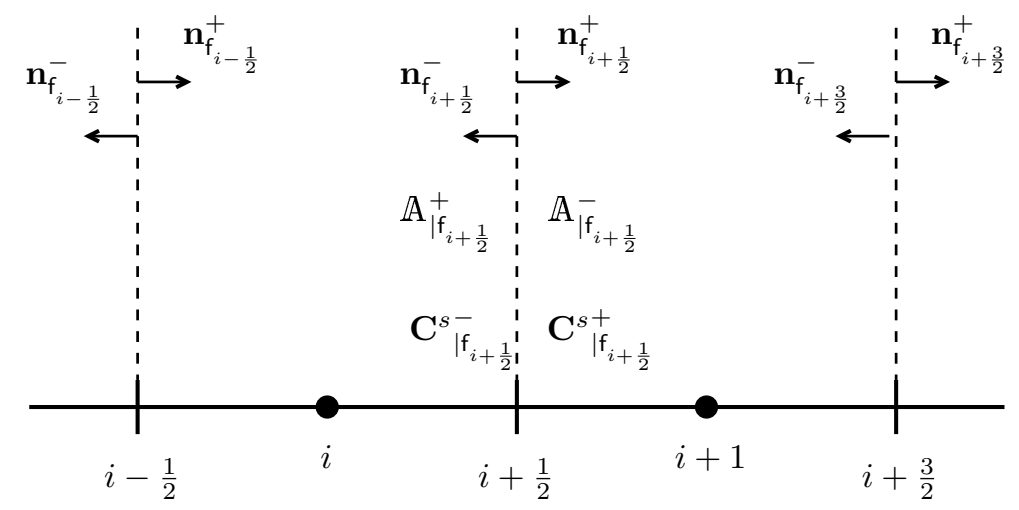

Fig. 1. Notation of the numerical flux functions along direction $x$. To ease the notation, we do not show the indices $j$ and $k$. We denote the unit normal vector to face $\mathrm{f}_{\ell}$ by $\boldsymbol{n}_{\mathrm{f}_{\ell}}, \ell=i \pm \frac{1}{2}, i+\frac{3}{2}$. Vectors $\boldsymbol{n}_{\mathrm{f}_{\ell}}^{+}$are oriented along the positive real axis (from left to right); vectors $\boldsymbol{n}_{\mathrm{f}_{\ell}}^{-}$are in the opposite sense. Similarly, at interface $\mathbf{f}_{i+\frac{1}{2}}$ matrix $\mathbb{A}_{x}=\mathbb{A}_{x}^{+}+\mathbb{A}_{x}^{-}$is decomposed into characteristics waves traveling from left to right, $\mathbb{A}_{x}^{+}$, which transport solution $\mathbf{C}^{s-}$ from inside cell $i$ toward the cell interface, and from right to left, $\mathbb{A}_{x}^{-}$, which transport the solution $\mathbf{C}^{s+}$ from inside cell $i+1$ towards the cell interface. Let $\mathbf{C}^{s \pm} \mathrm{f}_{i+\frac{1}{2}}$ be a shortcut for $\mathbf{C}^{s}\left(x_{i+\frac{1}{2}}^{ \pm}, y, z, t\right)$. According to this notation, the upwind flux is given by $\left.\widehat{\mathbb{A}_{x} \mathbf{C}^{s}}\right|_{\mathrm{f}_{i+\frac{1}{2}}}=\mathbb{A}_{x}^{+} \mathbf{C}_{\mid \mathrm{f}_{i+\frac{1}{2}}}+\mathbb{A}_{x}^{-} \mathbf{C}_{\mid \mathrm{f}_{i+\frac{1}{2}}+}$.

$$
\begin{aligned}
& {\left[\mathcal{I}^{x, s}\right]_{n, m, p}:=\int_{I}\left[\mathbb{A}_{x} \mathbf{C}^{s}(\boldsymbol{x}, t)\right]_{n, m, p} \frac{\partial \varphi^{I, l}(\boldsymbol{x})}{\partial x} d \boldsymbol{x}} \\
& =\sum_{l^{\prime}=1}^{N_{l}}\left(\boldsymbol{\Phi}_{x}\right)_{l, l^{\prime}}\left(\alpha_{x}^{s} \sqrt{\frac{n+1}{2}} C_{n+1, m, p}^{s, I, l^{\prime}}(t)+\alpha_{x}^{s} \sqrt{\frac{n}{2}} C_{n-1, m, p}^{s, I, l^{\prime}}(t)+u_{x}^{s} C_{n, m, p}^{s, I, l^{\prime}}(t)\right), \\
& {\left[\mathcal{I}^{y, s}\right]_{n, m, p}:=\int_{I}\left[\mathbb{A}_{y} \mathbf{C}^{s}(\boldsymbol{x}, t)\right]_{n, m, p} \frac{\partial \varphi^{I, l}(\boldsymbol{x})}{\partial y} d \boldsymbol{x}} \\
& =\sum_{l^{\prime}=1}^{N_{l}}\left(\boldsymbol{\Phi}_{y}\right)_{l, l^{\prime}}\left(\alpha_{y}^{s} \sqrt{\frac{m+1}{2}} C_{n, m+1, p}^{s, I, l^{\prime}}(t)+\alpha_{y}^{s} \sqrt{\frac{m}{2}} C_{n, m-1, p}^{s, I, l^{\prime}}(t)+u_{y}^{s} C_{n, m, p}^{s, I, l^{\prime}}(t)\right), \\
& {\left[\mathcal{I}^{z, s}\right]_{n, m, p}:=\int_{I}\left[\mathbb{A}_{z} \mathbf{C}^{s}(\boldsymbol{x}, t)\right]_{n, m, p} \frac{\partial \varphi^{I, l}(\boldsymbol{x})}{\partial z} d \boldsymbol{x}} \\
& =\sum_{l^{\prime}=1}^{N_{l}}\left(\boldsymbol{\Phi}_{z}\right)_{l, l^{\prime}}\left(\alpha_{z}^{s} \sqrt{\frac{p+1}{2}} C_{n, m, p+1}^{s, I, l^{\prime}}(t)+\alpha_{z}^{s} \sqrt{\frac{p}{2}} C_{n, m, p-1}^{s, I, l^{\prime}}(t)+u_{z}^{s} C_{n, m, p}^{s, I, l^{\prime}}(t)\right),
\end{aligned}
$$

where, for future reference, we introduced the symbols $\mathcal{I}^{\beta, s}$. After splitting the integral in (34) in the onedimensional integrals for the Legendre polynomials, formulas (24a) and (24b) can easily be applied.

On the other hand, the calculation of the boundary integral term of (32) deserves special attention since we need to introduce upwind numerical flux functions. The choice of upwinding the fluxes is dictated by considerations on the numerical stability of the scheme, $c f$. [35], since upwinding make the proper information propagation possible in the computational domain. Figure 1 illustrates the meaning of the main symbols that we adopt in rest of the section and throughout the paper. In view of the face orientation and noting that $n_{\beta}$ can only be \pm 1 or zero, the total fluxes along the directions $x, y$, and $z$ are given by 


$$
\begin{aligned}
\delta_{x}\left[\left[\mathrm{~A}_{x} \mathbf{C}^{s}(\boldsymbol{x}, t)\right]_{n, m, p} \varphi^{I, l}(\boldsymbol{x})\right]_{\partial I_{x}} & =\int_{\mathrm{f}_{i+\frac{1}{2}, j, k}}\left[\mathbb{A}_{x} \mathbf{C}^{s}\left(x_{i+\frac{1}{2}}, y, z, t\right)\right]_{n, m, p} \varphi^{I, l}\left(x_{i+\frac{1}{2}}, y, z\right) d y d z \\
& -\int_{\mathrm{f}_{i-\frac{1}{2}, j, k}}\left[\mathbb{A}_{x} \mathbf{C}^{s}\left(x_{i-\frac{1}{2}}, y, z, t\right)\right]_{n, m, p} \varphi^{I, l}\left(x_{i-\frac{1}{2}}, y, z\right) d y d z, \\
\delta_{y}\left[\left[\mathbb{A}_{y} \mathbf{C}^{s}(\boldsymbol{x}, t)\right]_{n, m, p} \varphi^{I, l}(\boldsymbol{x})\right]_{\partial I_{y}} & =\int_{\mathrm{f}_{i, j+\frac{1}{2}, k}}\left[\mathbb{A}_{y} \mathbf{C}^{s}\left(x, y_{j+\frac{1}{2}}, z, t\right)\right]_{n, m, p} \varphi^{I, l}\left(x, y_{j+\frac{1}{2}}, z\right) d x d z \\
& -\int_{\mathbf{f}_{i, j-\frac{1}{2}, k}}\left[\mathbb{A}_{y} \mathbf{C}^{s}\left(x, y_{j-\frac{1}{2}}, z, t\right)\right]_{n, m, p} \varphi^{I, l}\left(x, y_{j-\frac{1}{2}}, z\right) d x d z, \\
\delta_{z}\left[\left[\mathbb{A}_{z} \mathbf{C}^{s}(\boldsymbol{x}, t)\right]_{n, m, p} \varphi^{I, l}(\boldsymbol{x})\right]_{\partial I_{z}} & =\int_{\mathrm{f}_{i, j, k+\frac{1}{2}}}\left[\mathbb{A}_{z} \mathbf{C}^{s}\left(x, y, z_{j+\frac{1}{2}}, t\right)\right]_{n, m, p} \varphi^{I, l}\left(x, y, z_{j+\frac{1}{2}}\right) d x d y \\
& -\int_{\mathbf{f}_{i, j, k-\frac{1}{2}}}\left[\mathbb{A}_{z} \mathbf{C}^{s}\left(x, y, z_{j-\frac{1}{2}}, t\right)\right]_{n, m, p} \varphi^{I, l}\left(x, y, z_{j-\frac{1}{2}}\right) d x d y .
\end{aligned}
$$

Let $\mathbf{C}^{s}\left(x_{i+\frac{1}{2}}^{+}, y, z, t\right)$ denote $\lim _{\epsilon \rightarrow 0^{+}} \mathbf{C}^{s}\left(x_{i+\frac{1}{2}}+\epsilon, y, z, t\right)$, and similarly for the trace at the other faces. The integral on face $\mathrm{f}_{i+\frac{1}{2}, j, k}$ is approximated by,

$$
\delta_{x}^{s,+}:=\int_{\mathbf{f}_{i+\frac{1}{2}, j, k}}\left[\widehat{\mathbb{A}_{x} \mathbf{C}^{s}}\left(x_{i+\frac{1}{2}}, y, z, t\right)\right]_{n, m, p} \varphi^{I, l}\left(x_{i+\frac{1}{2}}, y, z\right) d y d z,
$$

and the upwind numerical flux in the integral argument is defined as

$$
\widehat{\mathbf{A}_{x} \mathbf{C}^{s}}\left(x_{i+\frac{1}{2}}, y, z, t\right)=\underbrace{\mathbb{A}_{x}^{+} \mathbf{C}^{s}\left(x_{i+\frac{1}{2}}^{-}, y, z, t\right)}_{\text {from cell } I=I_{i, j, k}}+\underbrace{\mathbb{A}_{x}^{-} \mathbf{C}^{s}\left(x_{i+\frac{1}{2}}^{+}, y, z, t\right)}_{\text {from cell } I^{+}=I_{i+1, j, k}} .
$$

In this formula, we outlined the contributions from the two cells sharing the face $\mathrm{f}_{i+\frac{1}{2}, j, k}$, namely $I=I_{i, j, k}$ and $I^{+}:=I_{i+1, j, k}$, and denoted the upwind matrices given by the characteristic decomposition of matrix $\mathbb{A}_{x}$ by $\mathbb{A}_{x}^{+}$and $\mathbb{A}_{x}^{-}$. We recall that matrix $\mathbb{A}^{+}$is built from the positive eigenvalues of matrix $\mathbb{A}$, and matrix $\mathbb{A}^{-}$from the negative ones, so that $\mathbb{A}=\mathbb{A}^{+}+\mathbb{A}^{-}$. We refer to [61] for a presentation of this topic, while we discuss the major details of how to implement this algorithm efficiently in Remark 3.4 at the end of the section. Similarly, the numerical fluxes at the six faces $\mathrm{f}_{i \pm \frac{1}{2}, j, k}, \mathrm{f}_{i, j \pm \frac{1}{2}, k}$, and $\mathrm{f}_{i, j, k \pm \frac{1}{2}}$ are given by

$$
\begin{aligned}
& \left.\widehat{\mathbf{A}_{x} \mathbf{C}^{s}}\right|_{\mathrm{f}_{i \pm \frac{1}{2}, j, k}}=\mathbb{A}_{x}^{ \pm} \mathbf{C}^{s}\left(x_{i \pm \frac{1}{2}}^{\mp}, y, z, t\right)+\mathbb{A}_{x}^{\mp} \mathbf{C}^{s}\left(x_{i \pm \frac{1}{2}}^{ \pm}, y, z, t\right), \\
& \left.\widehat{\mathbb{A}_{y} \mathbf{C}^{s}}\right|_{\mathrm{f}_{i, j \pm \frac{1}{2}, k}}=\mathbb{A}_{y}^{ \pm} \mathbf{C}^{s}\left(x, y_{j \pm \frac{1}{2}}^{\mp}, z, t\right)+\mathbb{A}_{y}^{\mp} \mathbf{C}^{s}\left(x, y_{j \pm \frac{1}{2}}^{ \pm}, z, t\right), \\
& \left.\widehat{\mathbb{A}_{z} \mathbf{C}^{s}}\right|_{\mathfrak{f}_{i, j, k \pm \frac{1}{2}}}=\mathbb{A}_{z}^{ \pm} \mathbf{C}^{s}\left(x, y, z_{k \pm \frac{1}{2}}^{\mp}, t\right)+\mathbb{A}_{z}^{\mp} \mathbf{C}^{s}\left(x, y, z_{k \pm \frac{1}{2}}^{ \pm}, t\right),
\end{aligned}
$$

where $\mathbb{A}_{x}^{ \pm}, \mathbb{A}_{y}^{ \pm}$, and $\mathbb{A}_{z}^{ \pm}$are the upwind matrices given by the characteristic decomposition of matrices $\mathbb{A}_{x}$, $\mathbb{A}_{y}, \mathbb{A}_{z}$, respectively. In the following, we continue detailing the procedure for the numerical flux across face $\mathrm{f}_{i+\frac{1}{2}, j, k}$, the extension to the other five faces being deemed straightforward. Using the expansion of the Hermite coefficients in the local polynomial basis functions, we can rewrite the contribution from cell $I=I_{i, j, k}$ in (38) as follows, 


$$
\begin{array}{rll}
{\left[\mathbb{A}_{x}^{+} \mathbf{C}^{s}\left(x_{i+\frac{1}{2}}^{-}, y, z, t\right)\right]_{n, m, p}} & =\sum_{n^{\prime}=0}^{N_{v_{x}}}\left(\boldsymbol{A}_{x}^{+}\right)_{n+1, n^{\prime}+1} C_{n^{\prime}, m, p}^{s}\left(x_{i+\frac{1}{2}}^{-}, y, z, t\right) & \text { [since } \left.x_{i+\frac{1}{2}}^{-} \in I\right] \\
& =\sum_{n^{\prime}=0}^{N_{v_{x}}}\left(\boldsymbol{A}_{x}^{+}\right)_{n+1, n^{\prime}+1} \sum_{l^{\prime}=1}^{N_{l}} C_{n^{\prime}, m, p}^{s, I, l^{\prime}}(t) \varphi^{I, l^{\prime}}\left(x_{i+\frac{1}{2}}^{-}, y, z\right) \quad \text { [rearrange the summation] } \\
& =\sum_{l^{\prime}=1}^{N_{l}}\left(\sum_{n^{\prime}=0}^{N_{v_{x}}}\left(\boldsymbol{A}_{x}^{+}\right)_{n+1, n^{\prime}+1} C_{n^{\prime}, m, p}^{s, l, l^{\prime}}(t)\right) \varphi^{I, l^{\prime}}\left(x_{i+\frac{1}{2}}^{-}, y, z\right) .
\end{array}
$$

Analogously, using the local polynomials $\varphi^{I^{+}, l^{\prime}}$ defined on cell $I^{+}$,

$$
\begin{array}{rlrl}
{\left[\mathbb{A}_{x}^{-} \mathbf{C}^{s}\left(x_{i+\frac{1}{2}}^{+}, y, z, t\right)\right]_{n, m, p}} & =\sum_{n^{\prime}=0}^{N_{v_{x}}}\left(\boldsymbol{A}_{x}^{-}\right)_{n+1, n^{\prime}+1} C_{n^{\prime}, m, p}^{s}\left(x_{i+\frac{1}{2}}^{+}, y, z, t\right) & & \text { [since } \left.x_{i+\frac{1}{2}}^{+} \in I^{+}\right] \\
& =\sum_{n^{\prime}=0}^{N_{v_{x}}}\left(\boldsymbol{A}_{x}^{-}\right)_{n+1, n^{\prime}+1} \sum_{l^{\prime}=1}^{N_{l}} C_{n^{\prime}, m, p}^{s, I^{+}, l^{\prime}}(t) \varphi^{I^{+}, l^{\prime}}\left(x_{i+\frac{1}{2}}^{+}, y, z\right) & \text { [rearrange the summation] } \\
& =\sum_{l^{\prime}=1}^{N_{l}}\left(\sum_{n^{\prime}=0}^{N_{v_{x}}}\left(\boldsymbol{A}_{x}^{-}\right)_{n+1, n^{\prime}+1} C_{n^{\prime}, m, p}^{s, I^{+}, l^{\prime}}(t)\right) \varphi^{I^{+}, l^{\prime}}\left(x_{i+\frac{1}{2}}^{+}, y, z\right) .
\end{array}
$$

The matrices $\boldsymbol{A}_{x}^{ \pm}$corresponding to the linear operators $\mathbb{A}_{x}^{ \pm}$are implemented as explained in Remark 3.4 and are indexed from 1 to $N_{v_{x}}+1$. We compute $\delta_{x}^{s,+}$ using (37) and splitting (38), namely

$$
\begin{aligned}
\delta_{x}^{s,+}= & \sum_{l^{\prime}=1}^{N_{l}}\left(\sum_{n^{\prime}=0}^{N_{v_{x}}}\left(\boldsymbol{A}_{x}^{+}\right)_{n+1, n^{\prime}+1} C_{n^{\prime}, m, p}^{s, I, l^{\prime}}(t)\right) \underbrace{\left(\int_{\mathrm{f}_{i+\frac{1}{2}, j, k}} \varphi^{I, l^{\prime}}\left(x_{i+\frac{1}{2}}^{-}, y, z\right) \varphi^{I, l}\left(x_{i+\frac{1}{2}}^{-}, y, z\right) d y d z\right)}_{\text {only cell } I} \\
& +\sum_{l^{\prime}=1}^{N_{l}}\left(\sum_{n^{\prime}=0}^{N_{v_{x}}}\left(\boldsymbol{A}_{x}^{-}\right)_{n+1, n^{\prime}+1} C_{n^{\prime}, m, p}^{s, I^{+}, l^{\prime}}(t)\right) \underbrace{\left(\int_{\mathrm{f}_{i+\frac{1}{2}, j, k}} \varphi^{I^{+}, l^{\prime}}\left(x_{i+\frac{1}{2}}^{+}, y, z\right) \varphi^{I, l}\left(x_{i+\frac{1}{2}}^{-}, y, z\right) d y d z\right)}_{\text {two distinct cells } I \text { and } I^{+}} .
\end{aligned}
$$

We compute the face integrals in (40) by using orthogonality properties of the Legendre polynomials (24a). To summarize, the local spectral Hermite-DG discretization of the linear term of the Vlasov equation reads

$$
\int_{I} \mathcal{L}_{n, m, p}\left(C^{s}\right) \varphi^{I, l}(\boldsymbol{x}) d \boldsymbol{x}=\sum_{\beta \in\{x, y, z\}}\left(-\left[\mathcal{I}^{\beta}\right]_{n, m, p}+\left(\delta_{\beta}^{s,+}-\delta_{\beta}^{s,-}\right)\right)
$$

where the volume terms $\left[\mathcal{I}^{x}\right]_{n, m, p},\left[\mathcal{I}^{y}\right]_{n, m, p}$ and $\left[\mathcal{I}^{z}\right]_{n, m, p}$ are defined in (35a), (35b) and (35c), respectively. The boundary term $\delta_{x}^{s,+}$ defined in (37) is computed as in (40), while similar derivations can be carried out for the terms $\delta_{x}^{s,-}, \delta_{y}^{s, \pm}, \delta_{z}^{s, \pm}$.

Remark 3.3 The upwind integrated fluxes given by combining (36a)-(36c) and (39a)-(39c) are equivalent to solving the Riemann problems defined by the discontinuous traces of the Hermite coefficients at the cell interfaces for the linear hyperbolic system (16), see, e.g., [61].

Remark 3.4 For an efficient implementation of (39a), (39b), (39c), the vector $\mathbf{C}^{s}$ of the Hermite coeffcients is stored as a $3 r d$ order tensor. The implemented matrices $\boldsymbol{A}_{\beta} \in \mathbb{R}^{\left(N_{v_{\beta}}+1\right) \times\left(N_{v_{\beta}}+1\right)}$ corresponding to the linear operators $\mathbb{A}_{\beta}$ for $\beta \in\{x, y, z\}$, are real, symmetric, and tridiagonal with entries, 


$$
\begin{array}{rlrl}
\left(\boldsymbol{A}_{\beta}\right)_{i, i} & =u_{\beta}^{s}, & & \text { for } \quad i=1, \ldots, N_{v_{\beta}}+1, \\
\left(\boldsymbol{A}_{\beta}\right)_{i, i-1} & =\alpha_{\beta}^{s} \sqrt{(i-1) / 2}, & \text { for } \quad i=2, \ldots, N_{v_{\beta}}+1, \\
\left(\boldsymbol{A}_{\beta}\right)_{i, i+1} & =\alpha_{\beta}^{s} \sqrt{i / 2}, & & \text { for } \quad i=1, \ldots, N_{v_{\beta}} .
\end{array}
$$

The matrices $\left\{\boldsymbol{A}_{\beta}\right\}_{\beta \in\{x, y, z\}}$ can be diagonalized, namely there exists an orthogonal matrix $\mathbf{R}_{\beta}$ (i.e., $\mathbf{R}_{\beta}^{T}=$ $\mathbf{R}_{\beta}^{-1}$ ), whose columns are the eigenvectors of matrix $\boldsymbol{A}_{\beta}$ and a diagonal matrix $\mathbf{D}_{\beta}$, whose diagonal elements $\left\{\lambda_{\beta, i}\right\}_{i=1}^{N_{\beta}}$ are the eigenvalues of $\boldsymbol{A}_{\beta}$. Therefore, it holds that $\boldsymbol{A}_{\beta}=\mathbf{R}_{\beta}^{T} \mathbf{D}_{\beta} \mathbf{R}_{\beta}$, and we can define the "upwind" matrices $\boldsymbol{A}_{\beta}^{ \pm}=\mathbf{R}_{\beta}^{T} \mathbf{D}_{\beta}^{ \pm} \mathbf{R}_{\beta}$, where $\mathbf{D}_{\beta}^{ \pm}=\frac{1}{2} \operatorname{diag}\left(\lambda_{\beta, i} \pm\left|\lambda_{\beta, i}\right|\right)$. Note that the computation of the eigenvalues and eigenvectors of the matrices $\left\{\boldsymbol{A}_{\beta}\right\}$ is not computationally expensive: the matrices are tridiagonal, do not change at each mesh interface, and are independent of time. Consequently, they only need to be computed once for a single cell and stored in memory at the beginning of each numerical simulation.

\subsection{Nonlinear terms}

Consider the local contribution of the nonlinear terms in the semi-discretization (20) of Vlasov equation, namely

$$
\int_{I} \mathcal{N}_{n, m, p}\left(C^{s}\right) \varphi^{I, l}(\boldsymbol{x}) d \boldsymbol{x}
$$

The electric and magnetic field $\boldsymbol{E}$ and $\boldsymbol{B}$, which are involved in these terms, are approximated using piecewise polynomials. In particular, in the mesh element $I$ we consider the multivariate Legendre polynomial basis $\left\{\varphi^{I, l}(\boldsymbol{x})\right\}_{l=1}^{N_{l}}$. To ease the exposition, we assume that the degree of the local polynomial spaces are the same, so that,

$$
\begin{gathered}
\boldsymbol{E}^{N}(\boldsymbol{x}, t)=\left(\begin{array}{l}
E_{x}^{N}(\boldsymbol{x}, t) \\
E_{y}^{N}(\boldsymbol{x}, t) \\
E_{z}^{N}(\boldsymbol{x}, t)
\end{array}\right)=\left(\begin{array}{c}
\sum_{I, l} E_{x}^{I, l}(t) \varphi^{I, l}(\boldsymbol{x}) \\
\sum_{I, l} E_{y}^{I, l}(t) \varphi^{I, l}(\boldsymbol{x}) \\
\sum_{I, l} E_{z}^{I, l}(t) \varphi^{I, l}(\boldsymbol{x})
\end{array}\right)=\sum_{I, l}\left(\begin{array}{l}
E_{x}^{I, l}(t) \\
E_{y}^{I, l}(t) \\
E_{z}^{I, l}(t)
\end{array}\right) \varphi^{I, l}(\boldsymbol{x})=: \sum_{I, l} \boldsymbol{E}^{I, l}(t) \varphi^{I, l}(\boldsymbol{x}), \\
\boldsymbol{B}^{N}(\boldsymbol{x}, t)=\left(\begin{array}{l}
B_{x}^{N}(\boldsymbol{x}, t) \\
B_{y}^{N}(\boldsymbol{x}, t) \\
B_{z}^{N}(\boldsymbol{x}, t)
\end{array}\right)=\left(\begin{array}{c}
\sum_{I, l} B_{x}^{I, l}(t) \varphi^{I, l}(\boldsymbol{x}) \\
\sum_{I, l} B_{y}^{I, l}(t) \varphi^{I, l}(\boldsymbol{x}) \\
\sum_{I, l} B_{z}^{I, l}(t) \varphi^{I, l}(\boldsymbol{x})
\end{array}\right)=\sum_{I, l}\left(\begin{array}{c}
B_{x}^{I, l}(t) \\
B_{y}^{I, l}(t) \\
B_{z}^{I, l}(t)
\end{array}\right) \varphi^{I, l}(\boldsymbol{x})=: \sum_{I, l} \boldsymbol{B}^{I, l}(t) \varphi^{I, l}(\boldsymbol{x}) .
\end{gathered}
$$

To keep the presentation focused we only present the spatial discretization of the terms involving the electric and magnetic fields in the $x$-direction. In the other directions the derivation follows straightforwardly.

From (20), we define

$$
\left[\mathcal{I}^{E_{x}, s}\right]_{n, m, p}:=\frac{q^{s}}{m^{s}} \frac{\omega_{c e}}{\omega_{p e}} \int_{I} \frac{\sqrt{2 n}}{\alpha_{x}^{s}} E_{x}^{N}(\boldsymbol{x}, t) C_{n-1, m, p}^{s}(\boldsymbol{x}, t) \varphi^{I, l}(\boldsymbol{x}) d \boldsymbol{x} .
$$

Using the spatial DG discretization of the Hermite coefficients and the electric field yields,

$$
\begin{aligned}
{\left[\mathcal{I}^{E_{x}, s}\right]_{n, m, p} } & =\frac{q^{s}}{m^{s}} \frac{\omega_{c e}}{\omega_{p e}} \frac{\sqrt{2 n}}{\alpha_{x}^{s}} \sum_{l^{\prime}=1}^{N_{l}} C_{n-1, m, p}^{s, I, l^{\prime}}(t) \int_{I} E_{x}^{N}(\boldsymbol{x}, t) \varphi^{I, l^{\prime}}(\boldsymbol{x}) \varphi^{I, l}(\boldsymbol{x}) d \boldsymbol{x} \\
& =\frac{q^{s}}{m^{s}} \frac{\omega_{c e}}{\omega_{p e}} \frac{\sqrt{2 n}}{\alpha_{x}^{s}} \sum_{l^{\prime}=1}^{N_{l}} C_{n-1, m, p}^{s, I, l^{\prime}}(t) \sum_{l^{\prime \prime}=1}^{N_{l}} E_{x}^{I, l^{\prime \prime}}(t) \int_{I} \varphi^{I, l^{\prime \prime}}(\boldsymbol{x}) \varphi^{I, l^{\prime}}(\boldsymbol{x}) \varphi^{I, l}(\boldsymbol{x}) d \boldsymbol{x},
\end{aligned}
$$

where the integral appearing in the last term can be computed using formula (24c). We define and compute the other nonlinear terms $\left[\mathcal{I}^{E_{y}, s}\right]_{n, m, p}$ and $\left[\mathcal{I}^{E_{z}, s}\right]_{n, m, p}$ involving $E_{y}$ and $E_{z}$, respectively, in the same manner. 
To deal with the terms involving the magnetic field $\boldsymbol{B}$, we define

$$
\begin{gathered}
{\left[\mathcal{I}^{B_{x}, s}\right]_{n, m, p}:=\frac{q^{s}}{m^{s}} \frac{\omega_{c e}}{\omega_{p e}} \int_{I} B_{x}^{N}(\boldsymbol{x}, t)\left(\sqrt{m p}\left(\frac{\alpha_{z}^{s}}{\alpha_{y}^{s}}-\frac{\alpha_{y}^{s}}{\alpha_{z}^{s}}\right) C_{n, m-1, p-1}^{s}+\sqrt{m(p+1)} \frac{\alpha_{z}^{s}}{\alpha_{y}^{s}} C_{n, m-1, p+1}^{s}\right.} \\
\left.-\sqrt{(m+1) p} \frac{\alpha_{y}^{s}}{\alpha_{z}^{s}} C_{n, m+1, p-1}^{s}+\sqrt{2 m} \frac{u_{z}^{s}}{\alpha_{y}^{s}} C_{n, m-1, p}^{s}-\sqrt{2 p} \frac{u_{y}^{s}}{\alpha_{z}^{s}} C_{n, m, p-1}^{s}\right) \varphi^{I, l}(\boldsymbol{x}) d \boldsymbol{x},
\end{gathered}
$$

and we introduce the quantity

$$
\begin{aligned}
\zeta_{x, n, m, p}^{s, I, l^{\prime}}(t):= & \sqrt{m p}\left(\frac{\alpha_{z}^{s}}{\alpha_{y}^{s}}-\frac{\alpha_{y}^{s}}{\alpha_{z}^{s}}\right) C_{n, m-1, p-1}^{s, I, l^{\prime}}(t)+\sqrt{m(p+1)} \frac{\alpha_{z}^{s}}{\alpha_{y}^{s}} C_{n, m-1, p+1}^{s, I, l^{\prime}}(t) \\
& -\sqrt{(m+1) p} \frac{\alpha_{y}^{s}}{\alpha_{z}^{s}} C_{n, m+1, p-1}^{s, I, l^{\prime}}(t)+\sqrt{2 m} \frac{u_{z}^{s}}{\alpha_{y}^{s}} C_{n, m-1, p}^{s, I, l^{\prime}}(t)-\sqrt{2 p} \frac{u_{y}^{s}}{\alpha_{z}^{s}} C_{n, m, p-1}^{s, I, l^{\prime}}(t) .
\end{aligned}
$$

Expanding the magnetic field $B_{x}$ in the DG basis, one gets

$$
\begin{aligned}
{\left[\mathcal{I}^{B_{x}, s}\right]_{n, m, p} } & =\frac{q^{s}}{m^{s}} \frac{\omega_{c e}}{\omega_{p e}} \int_{I} B_{x}^{N}(\boldsymbol{x}, t) \sum_{l^{\prime}=1}^{N_{l}} \zeta_{x, n, m, p}^{s, I, l^{\prime}}(t) \varphi^{I, l^{\prime}}(\boldsymbol{x}) \varphi^{I, l}(\boldsymbol{x}) d \boldsymbol{x} \\
& =\frac{q^{s}}{m^{s}} \frac{\omega_{c e}}{\omega_{p e}} \sum_{l^{\prime}=1}^{N_{l}} \zeta_{x, n, m, p}^{s, I, l^{\prime}}(t) \sum_{l^{\prime \prime}=1}^{N_{l}} B_{x}^{I, l^{\prime \prime}}(t) \int_{I} \varphi^{I, l^{\prime \prime}}(\boldsymbol{x}) \varphi^{I, l^{\prime}}(\boldsymbol{x}) \varphi^{I, l}(\boldsymbol{x}) d \boldsymbol{x} .
\end{aligned}
$$

An analogous approach yields the approximation of the terms $\left[\mathcal{I}^{B_{y}, s}\right]_{n, m, p}$ and $\left[\mathcal{I}^{B_{z}, s}\right]_{n, m, p}$ involving $B_{y}$ and $B_{z}$, respectively.

To summarize, the local spectral Hermite-DG discretization of the nonlinear term of the Vlasov equation reads

$$
\int_{I} \mathcal{N}_{n, m, p}\left(C^{s}\right) \varphi^{I, l}(\boldsymbol{x}) d \boldsymbol{x}=-\sum_{\beta \in\{x, y, z\}}\left(\left[\mathcal{I}^{E_{\beta}, s}\right]_{n, m, p}+\left[\mathcal{I}^{B_{\beta}, s}\right]_{n, m, p}\right),
$$

where the terms involving the electric field are defined in (44) and those involving the magnetic field are defined in (45).

\section{DG method for Maxwell's equations}

In this section, we discuss the discontinuous Galerkin approximation of the Maxwell equations (2)-(3). In the continuum framework, the constraint equations (4) and (5) are satisfied at any time $t>0$ if they are satisfied at the initial time $t=0$. Since we do not approximate such equations directly, we expect that a violation occurs, i.e., the numerical approximations to the electromagnetic fields $\boldsymbol{E}, \boldsymbol{B}, \boldsymbol{J}$ and $\rho$ provided by the discontinuous Galerkin method satisfy them only in an approximate manner. However, this error is expected to be small and to decrease to zero at least at the same convergence rate of the numerical approximations used in the other two equations.

To perform the DG approximation of the Maxwell equations, we first reformulate them in the divergence form. It is worth noting that our approach differs from that proposed in [26, 27], where a local integration by parts is carried out on the curl terms. Starting from the divergence form, we, then, integrate by part on every element and apply the Gauss-Green divergence theorem according to the original DG method proposed in $[34,35,36]$. The local integration by part and the discontinuous nature of the approximate electromagnetic fields at the cell interface allows us to introduce the numerical flux functions, which can be central or upwind. The upwinding of the numerical flux is performed numerically, even though the associated Riemann problem can be easily solved exactly, cf. [26]. Such choice does not reduce the computational efficiency because the mesh is Cartesian and the few terms that are needed for the flux estimation can be precomputed and stored at negligible cost. 
Eqs. (2)-(3) in conservative form read as

$$
\frac{\partial \boldsymbol{u}}{\partial t}+\nabla_{\boldsymbol{x}} \cdot \mathbb{F}(\boldsymbol{u})=\boldsymbol{W}(\boldsymbol{u})
$$

where the components of the electric and magnetic fields form the vector of conservative unknowns $\boldsymbol{u}, \boldsymbol{W}$ represents the source terms, $\mathbb{F}(\boldsymbol{u})$ is the flux function, and the operator $\nabla_{\boldsymbol{x}} \cdot$ denotes the row-wise divergence. Using (2) and (3) we define

$$
\boldsymbol{u}:=\left(\begin{array}{l}
\boldsymbol{E} \\
\boldsymbol{B}
\end{array}\right) \in \mathbb{R}^{6}, \quad \boldsymbol{W}=\left(\begin{array}{l}
\boldsymbol{W}_{\boldsymbol{E}} \\
\boldsymbol{W}_{\boldsymbol{B}}
\end{array}\right):=-\frac{\omega_{p e}}{\omega_{c e}}\left(\begin{array}{l}
\boldsymbol{J} \\
\mathbf{0}
\end{array}\right) \in \mathbb{R}^{6},
$$

and

$$
\mathbb{F}(\boldsymbol{u}):=\left(\begin{array}{c}
\mathbb{F}_{\boldsymbol{E}}(\boldsymbol{u}) \\
\mathbb{F}_{\boldsymbol{B}}(\boldsymbol{u})
\end{array}\right) \in \mathbb{R}^{6 \times 3}, \quad \nabla_{\boldsymbol{x}} \cdot \mathbb{F}(\boldsymbol{u})=\left(\begin{array}{c}
\nabla_{\boldsymbol{x}} \cdot \mathbb{F}_{\boldsymbol{E}}(\boldsymbol{u}) \\
\nabla_{\boldsymbol{x}} \cdot \mathbb{F}_{\boldsymbol{B}}(\boldsymbol{u})
\end{array}\right)=\left(\begin{array}{c}
-\nabla_{\boldsymbol{x}} \times \boldsymbol{B} \\
\nabla_{\boldsymbol{x}} \times \boldsymbol{E}
\end{array}\right) .
$$

We partition the vector flux $\mathbb{F}(\boldsymbol{u})$ in a columnwise form so that

$$
\nabla_{\boldsymbol{x}} \cdot \mathbb{F}(\boldsymbol{u})=\frac{\partial}{\partial x} \mathbf{F}_{x}(\boldsymbol{u})+\frac{\partial}{\partial y} \mathbf{F}_{y}(\boldsymbol{u})+\frac{\partial}{\partial z} \mathbf{F}_{z}(\boldsymbol{u}) .
$$

The terms $\mathbf{F}_{x}(\boldsymbol{u}), \mathbf{F}_{y}(\boldsymbol{u})$, and $\mathbf{F}_{z}(\boldsymbol{u})$ can be written via flux matrices $\mathbb{F}_{x}, \mathbb{F}_{y}, \mathbb{F}_{z} \in \mathbb{R}^{6 \times 6}$ as follows,

$$
\begin{aligned}
& \mathbf{F}_{x}(\boldsymbol{u})=\left(\begin{array}{c}
-\boldsymbol{e}_{x} \times \boldsymbol{B} \\
\boldsymbol{e}_{x} \times \boldsymbol{E}
\end{array}\right)=\left(\begin{array}{c}
0 \\
B_{z} \\
-B_{y} \\
\hline 0 \\
-E_{z} \\
E_{y}
\end{array}\right)=\left(\begin{array}{rrr|rrr}
0 & 0 & 0 \\
& & & 0 & -1 & 0 \\
\hline 0 & 0 & 0 & & \\
0 & 0 & -1 & & \\
0 & 1 & 0 &
\end{array}\right)\left(\begin{array}{c}
E_{x} \\
E_{y} \\
E_{z} \\
\hline B_{x} \\
B_{y} \\
B_{z}
\end{array}\right)=\mathbb{F}_{x} \boldsymbol{u} \\
& \mathbf{F}_{y}(\boldsymbol{u})=\left(\begin{array}{c}
-\boldsymbol{e}_{y} \times \boldsymbol{B} \\
\boldsymbol{e}_{y} \times \boldsymbol{E}
\end{array}\right)=\left(\begin{array}{c}
-B_{z} \\
0 \\
B_{x} \\
\hline E_{z} \\
0 \\
-E_{x}
\end{array}\right)=\left(\begin{array}{rrr|rrr} 
& & & 0 & 0 & -1 \\
& 0 & 0 & 0 \\
& & & 1 & 0 & 0 \\
\hline 0 & 0 & 1 & & \\
0 & 0 & 0 & & \\
-1 & 0 & 0 &
\end{array}\right)\left(\begin{array}{c}
E_{x} \\
E_{y} \\
E_{z} \\
\hline B_{x} \\
B_{y} \\
B_{z}
\end{array}\right)=\mathbb{F}_{y} \boldsymbol{u} \\
& \mathbf{F}_{z}(\boldsymbol{u})=\left(\begin{array}{c}
-\boldsymbol{e}_{z} \times \boldsymbol{B} \\
\boldsymbol{e}_{z} \times \boldsymbol{E}
\end{array}\right)=\left(\begin{array}{c}
B_{y} \\
-B_{x} \\
0 \\
\hline-E_{y} \\
E_{x} \\
0
\end{array}\right)=\left(\begin{array}{rrr|rrr} 
& & 0 & 1 & 0 \\
& & & 0 & 0 & 0 \\
\hline & -1 & 0 & & \\
1 & 0 & 0 & & \\
0 & 0 & 0 & &
\end{array}\right)\left(\begin{array}{l}
E_{x} \\
E_{y} \\
E_{z} \\
\hline B_{x} \\
B_{y} \\
B_{z}
\end{array}\right)=\mathbb{F}_{z} \boldsymbol{u},
\end{aligned}
$$

where vectors $\boldsymbol{e}_{x}=(1,0,0)^{T}, \boldsymbol{e}_{y}=(0,1,0)^{T}$, and $\boldsymbol{e}_{z}=(0,0,1)^{T}$ form the canonical basis of $\mathbb{R}^{3}$. Let $\boldsymbol{n}=\left(n_{x}, n_{y}, n_{z}\right)^{T}$ be a generic vector in $\mathbb{R}^{3}$. We denote $\mathbb{F}(\boldsymbol{u}) \boldsymbol{n}=\mathbf{F}_{x}(\boldsymbol{u}) n_{x}+\mathbf{F}_{y}(\boldsymbol{u}) n_{y}+\mathbf{F}_{z}(\boldsymbol{u}) n_{z}=$ $\sum_{\beta \in\{x, y, z\}} \boldsymbol{F}_{\beta}(\boldsymbol{u}) n_{\beta}$, and use the same definition for $\mathbb{F}_{\boldsymbol{E}}(\boldsymbol{u}) \boldsymbol{n}$ and $\mathbb{F}_{\boldsymbol{B}}(\boldsymbol{u}) \boldsymbol{n}$. A straightforward calculation shows that

$$
\mathbb{F}(\boldsymbol{u}) \boldsymbol{n}=\left(\begin{array}{c}
\mathbb{F}_{\boldsymbol{E}}(\boldsymbol{u}) \boldsymbol{n} \\
\mathbb{F}_{\boldsymbol{B}}(\boldsymbol{u}) \boldsymbol{n}
\end{array}\right)=\left(\begin{array}{r}
-\boldsymbol{n} \times \boldsymbol{B} \\
\boldsymbol{n} \times \boldsymbol{E}
\end{array}\right),
$$


so that

$$
\mathbb{F}_{\boldsymbol{E}}(\boldsymbol{u}) \boldsymbol{n} \cdot \boldsymbol{E}=\mathbb{F}_{\boldsymbol{B}}(\boldsymbol{u}) \boldsymbol{n} \cdot \boldsymbol{B}=\boldsymbol{n} \cdot(\boldsymbol{E} \times \boldsymbol{B}) \in \mathbb{R} .
$$

To derive the discontinuous Galerkin approximation, we multiply Eq. (46) by the local basis function $\varphi^{I, l}$ associated with cell $I$ and integrate over the spatial domain $\Omega_{x}$. Let $\boldsymbol{U}=\left(\boldsymbol{U}_{\boldsymbol{E}}^{T}, \boldsymbol{U}_{B}^{T}\right)^{T}$ denote the DG approximation of the vector-valued function $\boldsymbol{u}$ solution of the Maxwell equations. Since the support of $\varphi^{I, l}$ is given by cell $I$, we can restrict the integration to such cell. For $g \in\{\boldsymbol{E}, \boldsymbol{B}\}$,

$$
\int_{I} \frac{\partial \boldsymbol{U}_{g}}{\partial t}(\boldsymbol{x}, t) \varphi^{I, l}(\boldsymbol{x}) d \boldsymbol{x}+\int_{I} \nabla_{\boldsymbol{x}} \cdot \mathbb{F}_{g}(\boldsymbol{U}) \varphi^{I, l}(\boldsymbol{x}) d \boldsymbol{x}=\int_{I} \boldsymbol{W}_{g}^{N} \varphi^{I, l}(\boldsymbol{x}) d \boldsymbol{x},
$$

where $\boldsymbol{W}_{\boldsymbol{E}}^{N}:=-\omega_{p e} \boldsymbol{J}^{N} / \omega_{c e}$ and $\boldsymbol{W}_{\boldsymbol{B}}^{N}=\mathbf{0}$. Then, we integrate by parts the divergence term to get,

$$
\int_{I} \frac{\partial \boldsymbol{U}_{g}}{\partial t}(\boldsymbol{x}, t) \varphi^{I, l}(\boldsymbol{x}) d \boldsymbol{x}-\int_{I} \mathbb{F}_{g}(\boldsymbol{U}) \nabla_{\boldsymbol{x}} \varphi^{I, l}(\boldsymbol{x}) d \boldsymbol{x}+\int_{\partial I} \mathbb{F}_{g}(\boldsymbol{U}) \boldsymbol{n} \varphi^{I, l}(\boldsymbol{x}) d S=\int_{I} \boldsymbol{W}_{g}^{N} \varphi^{I, l}(\boldsymbol{x}) d \boldsymbol{x} .
$$

We substitute the expansions (42), (43) of the electric field $\boldsymbol{E}$ and the magnetic induction $\boldsymbol{B}$ in (56). The first term in (56) contains the time-derivatives of $\boldsymbol{E}^{I, l}(t)$ and $\boldsymbol{B}^{I, l}(t)$. A direct substitution and the orthogonality of Legendre functions $\varphi^{I, l}$ yields

$$
\int_{I} \frac{\partial \boldsymbol{U}(t)}{\partial t} \varphi^{I, l} d \boldsymbol{x}=\left(\begin{array}{l}
\sum_{l^{\prime}=1}^{N_{l}} \frac{d \boldsymbol{E}^{I, l^{\prime}}(t)}{d t} \int_{I} \varphi^{I, l^{\prime}} \varphi^{I, l} d \boldsymbol{x} \\
\sum_{l^{\prime}=1}^{N_{l}} \frac{d \boldsymbol{B}^{I, l^{\prime}}(t)}{d t} \int_{I} \varphi^{I, l^{\prime}} \varphi^{I, l} d \boldsymbol{x}
\end{array}\right)=\mu_{I, l} \frac{d \boldsymbol{U}^{I, l}(t)}{d t} \quad \text { with } \quad \boldsymbol{U}^{I, l}(t):=\left(\begin{array}{c}
\boldsymbol{E}^{I, l}(t) \\
\boldsymbol{B}^{I, l}(t)
\end{array}\right),
$$

where the multiplicative factor $\mu_{I, l}:=\int_{I}\left(\varphi^{I, l}\right)^{2} d \boldsymbol{x}$ is as in (27). The second term in (56) is the volume integral of the generalized vector flux function $\mathbb{F}(\boldsymbol{U})$, and entails computing the term

$$
\mathcal{I}_{F}:=\int_{I}\left(\mathbf{F}_{x}(\boldsymbol{U}) \frac{\partial \varphi^{I, l}(\boldsymbol{x})}{\partial x}+\mathbf{F}_{y}(\boldsymbol{U}) \frac{\partial \varphi^{I, l}(\boldsymbol{x})}{\partial y}+\mathbf{F}_{z}(\boldsymbol{U}) \frac{\partial \varphi^{I, l}(\boldsymbol{x})}{\partial z}\right) d \boldsymbol{x} .
$$

To reformulate each addendum in (58) as the product of an integral involving only the DG basis functions and a term with the DG coefficients $\boldsymbol{E}^{I, l}(t)$ and $\boldsymbol{B}^{I, l}(t)$, we split the volume integral into the three distinct contributions, for each $\beta \in\{x, y, z\}$. It holds

$$
\mathcal{I}_{F}=\sum_{\beta \in\{x, y, z\}} \sum_{l^{\prime}=1}^{N_{l}}\left[\int_{I} \frac{\partial \varphi^{I, l}(\boldsymbol{x})}{\partial \beta} \varphi^{I, l^{\prime}}(\boldsymbol{x}) d \boldsymbol{x}\right]\left(\begin{array}{r}
-\boldsymbol{e}_{\beta} \times \boldsymbol{B}^{I, l^{\prime}} \\
\boldsymbol{e}_{\beta} \times \boldsymbol{E}^{I, l^{\prime}}
\end{array}\right)=\sum_{\beta \in\{x, y, z\}} \sum_{l^{\prime}=1}^{N_{l}}\left(\boldsymbol{\Phi}_{\beta}\right)_{l, l^{\prime}}\left(\begin{array}{r}
-\boldsymbol{e}_{\beta} \times \boldsymbol{B}^{I, l^{\prime}} \\
\boldsymbol{e}_{\beta} \times \boldsymbol{E}^{I, l^{\prime}}
\end{array}\right),
$$

where the matrix $\boldsymbol{\Phi}_{\beta}$ is defined in (34). The third term in (56) is the integral at the cell boundary $\partial I$. At the six faces of $\partial I$, we approximate the flux with the numerical flux as follows

$$
\begin{aligned}
\left.\mathbf{F}_{x}(\boldsymbol{U})\right|_{\mathrm{f}_{i \pm \frac{1}{2}, j, k}} & \approx \widehat{\mathbb{F}_{x} \boldsymbol{U}}\left(\boldsymbol{x}_{i \pm \frac{1}{2}, j, k}, t\right), \\
\left.\mathbf{F}_{y}(\boldsymbol{U})\right|_{\mathrm{f}_{i, j \pm \frac{1}{2}, k}} & \approx \widehat{\mathbb{F}_{y} \boldsymbol{U}}\left(\boldsymbol{x}_{i, j \pm \frac{1}{2}, k}, t\right), \\
\left.\mathbf{F}_{x}(\boldsymbol{U})\right|_{\mathrm{f}_{i, j, k \pm \frac{1}{2}}} & \approx \widehat{\mathbb{F}_{z} \boldsymbol{U}}\left(\boldsymbol{x}_{i, j, k \pm \frac{1}{2}}, t\right) .
\end{aligned}
$$

The size of the matrices $\mathbb{F}_{x}, \mathbb{F}_{y}$, and $\mathbb{F}_{z}$ is $6 \times 6$, and again they are the same for all the mesh interfaces, $c f$. Figure 1, so they can be computed and stored once for a single cell at a negligible cost. We consider two different kind of numerical fluxes, the central numerical flux and the upwind numerical flux. We provide a detailed description of the numerical treatment of the boundary integral in the $x$ direction, and, in particular, at the face $\mathrm{f}_{i+\frac{1}{2}, j, k}$. The extension to the other directions and faces is deemed straightforward. Let $\boldsymbol{n}=$ $\left(n_{x}, n_{y}, n_{z}\right)^{T}$ be the unit vector that is orthogonal to the boundary of $I$. Noting that, by construction, $n_{x}= \pm 1, n_{y}=n_{z}=0$ on the two faces $\mathrm{f}_{i \pm \frac{1}{2}, j, k}$, we obtain, 


$$
\int_{\partial_{x} I} n_{x} \mathbf{F}_{x}(\boldsymbol{U}) \varphi^{I, l}(\boldsymbol{x}) d S=\int_{\mathrm{f}_{i+\frac{1}{2}, j, k}} \mathbf{F}_{x}(\boldsymbol{U}) \varphi^{I, l}\left(x_{i+\frac{1}{2}}, y, z\right) d y d z-\int_{\mathrm{f}_{i-\frac{1}{2}, j, k}} \mathbf{F}_{x}(\boldsymbol{U}) \varphi^{I, l}\left(x_{i-\frac{1}{2}}, y, z\right) d y d z
$$

- The central numerical flux is given by the formula:

$$
\left.\mathbf{F}_{x}(\boldsymbol{U})\right|_{\mathrm{f}_{i+\frac{1}{2}, j, k}} \approx \widehat{\mathbb{F}_{x} \boldsymbol{U}}\left(\boldsymbol{x}_{i+\frac{1}{2}, j, k}, t\right)=\mathbb{F}_{x} \frac{1}{2}\left(\boldsymbol{U}\left(\boldsymbol{x}_{i+\frac{1}{2}, j, k}^{-}, t\right)+\boldsymbol{U}\left(\boldsymbol{x}_{i+\frac{1}{2}, j, k}^{+}, t\right)\right) .
$$

The implementation is straightforward.

- The upwind numerical flux is based on the characteristic decomposition of the flux matrices: $\mathbb{F}_{\beta}=\mathbb{F}_{\beta}^{+}+\mathbb{F}_{\beta}^{-}$, for each $\beta \in\{x, y, z\}$. We perform such decomposition numerically using the same procedure described in Remark 3.4 for the matrices $\mathbb{A}_{\beta}$. On face $\mathrm{f}_{i+\frac{1}{2}, j, k}$, we approximate the flux integral using the upwind flux decomposition of the matrix $\mathbb{F}_{x}$ as follows

$$
\left.\mathbf{F}_{x}(\boldsymbol{U})\right|_{\mathrm{f}_{i+\frac{1}{2}, j, k}} \approx \widehat{\mathbb{F}_{x} \boldsymbol{U}}\left(\boldsymbol{x}_{i+\frac{1}{2}, j, k}, t\right)=\underbrace{\mathbb{F}_{x}^{+} \boldsymbol{U}\left(x_{i+\frac{1}{2}}^{-}, y, z, t\right)}_{\text {from cell } I=I_{i, j, k}}+\underbrace{\mathbb{F}_{x}^{-} \boldsymbol{U}\left(x_{i+\frac{1}{2}}^{+}, y, z, t\right)}_{\text {from cell } I^{+}=I_{i+1, j, k}} .
$$

Thus, the third integral in (56) can be approximated by

$$
\begin{aligned}
\int_{\mathfrak{f}_{i+\frac{1}{2}, j, k}} \widehat{\mathbb{F}_{x} \boldsymbol{U}}\left(x_{i+\frac{1}{2}}, y, z, t\right) \varphi^{I, l}\left(x_{i+\frac{1}{2}}, y, z\right) d y d z \\
=\mathbb{F}_{x}^{+} \sum_{l^{\prime}=1}^{N_{l}} \boldsymbol{U}^{I, l^{\prime}}(t) \underbrace{\int_{\mathrm{f}_{i+\frac{1}{2}, j, k}} \varphi^{I, l^{\prime}}\left(x_{i+\frac{1}{2}}, y, z\right) \varphi^{I, l}\left(x_{i+\frac{1}{2}}, y, z\right) d y d z}_{\text {only cell } I} \\
+\mathbb{F}_{x}^{-} \sum_{l^{\prime}=1}^{N_{l}} \boldsymbol{U}^{I^{+}, l^{\prime}}(t) \underbrace{\int_{\mathrm{f}_{i+\frac{1}{2}, j, k}} \varphi^{I^{+}, l^{\prime}}\left(x_{i+\frac{1}{2}}, y, z\right) \varphi^{I, l}\left(x_{i+\frac{1}{2}}, y, z\right) d y d z .}_{\text {two distinct cells } I \text { and } I^{+}}
\end{aligned}
$$

The semi-discrete DG scheme for the Maxwell equations reads

$$
\mu_{I, l} \frac{d \boldsymbol{U}^{I, l}(t)}{d t}-\sum_{\beta \in\{x, y, z\}} \sum_{l^{\prime}=1}^{N_{l}}\left(\boldsymbol{\Phi}_{\beta}\right)_{l, l^{\prime}}\left(\begin{array}{r}
-\boldsymbol{e}_{\beta} \times \boldsymbol{B}^{I, l^{\prime}}(t) \\
\boldsymbol{e}_{\beta} \times \boldsymbol{E}^{I, l^{\prime}}(t)
\end{array}\right)+\int_{\partial I} \widehat{\mathbb{F}(\boldsymbol{U}) \boldsymbol{n}} \varphi^{I, l} d S=\int_{I} \boldsymbol{W}^{N}(\boldsymbol{U}) \varphi^{I, l} d \boldsymbol{x} .
$$

The contribution at the mesh faces is given in (64) or (65) depending on the choice of the numerical flux, while the last term in (67) can be easily computed by applying a quadrature rule.

Alternatively, using the DG approximation of the electric current $\boldsymbol{J}$ given by

$$
\boldsymbol{J}^{N}(\boldsymbol{x}, t)=\sum_{s} q^{s} \int_{\Omega_{v}} \boldsymbol{v} f^{s, N}(\boldsymbol{x}, \boldsymbol{v}, t) d \boldsymbol{v}=\sum_{s} q^{s} \alpha_{x}^{s} \alpha_{y}^{s} \alpha_{z}^{s} \sum_{I, l} \varphi^{I, l}(\boldsymbol{x})\left(C_{0,0,0}^{s, I, l}\left(\begin{array}{c}
u_{x}^{s} \\
u_{y}^{s} \\
u_{z}^{s}
\end{array}\right)+\frac{1}{\sqrt{2}}\left(\begin{array}{c}
\alpha_{x}^{s} C_{1,0,0}^{s, I, l} \\
\alpha_{y}^{s} C_{0,1,0}^{s, I, l} \\
\alpha_{z}^{s} C_{0,0,1}^{s, I, l}
\end{array}\right)\right),
$$

the source term in (67) can be easily reduced to the volume integral of the polynomial basis functions, and still be computed by applying formula (24a) independently in each spatial direction. In an analogous way, the DG approximation of the charge density (6) reads

$$
\rho^{N}(\boldsymbol{x}, t)=\sum_{s} q^{s} \int_{\Omega_{v}} f^{s, N}(\boldsymbol{x}, \boldsymbol{v}, t) d \boldsymbol{v}=\sum_{s} q^{s} \alpha_{x}^{s} \alpha_{y}^{s} \alpha_{z}^{s} \sum_{I, l} C_{0,0,0}^{s, I, l} \varphi^{I, l}(\boldsymbol{x})
$$


We use the DG approximation $\rho^{N}$ in the statement of Theorem 5.2 to characterize the behavior of the (discrete) total momentum.

\section{Conservation properties of the semi-discrete Hermite-DG method for periodic boundary conditions}

We list here three theorems that fully characterize the behavior of number of particles, total momentum and total energy in the semi-discrete formulation, where, for simplicity, we focus on periodic boundary conditions. Their proofs are reported in the final Appendix B.

\subsection{Conservation of the number of particles}

The total number of particles $N^{t o t}(t)$ at any time $t$ in the semi-discrete formulation of the Vlasov-Maxwell equations is given by summing the number of particles $N^{s}(t)$ of species $s$ over all the species. Since the Vlasov-Maxwell system does not have any physical process that may transform particles of a species into particles of another species, the number of particles of each species is also conserved separately. We show the conservation of the total number of particles in the following theorem.

Theorem 5.1 (Conservation of the number of particles) Let $f^{s, N}(\boldsymbol{x}, \boldsymbol{v}, t)$ be the numerical solution of the semi-discrete Vlasov-Maxwell problem. Then, the total number of particles in the plasma, $\mathcal{N}^{\text {tot }}(t)$, and the number of particles of each plasma species $s, \mathcal{N}^{s}(t)$, which are respectively defined as

$$
\mathcal{N}^{t o t}(t)=\sum_{s} \mathcal{N}^{s}(t), \quad \text { and } \quad \mathcal{N}^{s}(t)=\sum_{I} \int_{I} \int_{\Omega_{v}} f^{s, N}(\boldsymbol{x}, \boldsymbol{v}, t) d \boldsymbol{x} d \boldsymbol{v} \quad \forall s
$$

are constant in time, i.e., $d \mathcal{N}^{\text {tot }} / d t=0$ and $d \mathcal{N}^{s} / d t=0$ for each species $s$.

\subsection{Conservation properties of the total momentum}

We define the discrete total momentum as the sum of two terms which represent the momentum from the plasma kinetics and the momentum from the electromagnetic fields, i.e., $\boldsymbol{P}^{t o t, N}=\boldsymbol{P}_{f}^{N}(t)+\boldsymbol{P}_{\boldsymbol{E} \times \boldsymbol{B}}^{N}(t)$, where

$$
\begin{aligned}
\boldsymbol{P}_{f}^{N}(t) & :=\sum_{s} m^{s} \sum_{I} \int_{I}\left(\int_{\Omega_{v}} f^{s, N}(\boldsymbol{x}, \boldsymbol{v}, t) \boldsymbol{v} d \boldsymbol{v}\right) d \boldsymbol{x}, \\
\boldsymbol{P}_{\boldsymbol{E} \times \boldsymbol{B}}^{N}(t) & :=\left(\frac{\omega_{c e}}{\omega_{p e}}\right)^{2} \sum_{I} \int_{I} \boldsymbol{E}^{N}(\boldsymbol{x}, t) \times \boldsymbol{B}^{N}(\boldsymbol{x}, t) d \boldsymbol{x} .
\end{aligned}
$$

The total momentum in the continuum framework is given by the same definitions above, but using the fields $f^{s}, \boldsymbol{E}$ and $\boldsymbol{B}$ instead of the discrete fields $f^{s, N}, \boldsymbol{E}^{N}$ and $\boldsymbol{B}^{N}$, and is conserved in the Vlasov-Maxwell system for periodic boundary conditions. We characterize the behavior in time of the discrete total momentum by the following theorem, which shows that a violation of the momentum conservation occurs. However, if the solution fields are sufficiently regular (at least continuous), such a violation is expected to be small according to the adopted space and time resolution and the order of the method.

Theorem 5.2 (Conservation of momentum) Let $f^{s, N}, \boldsymbol{E}^{N}$ and $\boldsymbol{B}^{N}$ be the numerical solution of the semi-discrete variational formulation of the Vlasov-Maxwell problem. Let $\boldsymbol{P}^{\text {tot, }, N}(t):=\boldsymbol{P}_{f}^{N}(t)+\boldsymbol{P}_{\boldsymbol{E} \times \boldsymbol{B}}^{N}(t)$ be the discrete total momentum, where $\boldsymbol{P}_{f}^{N}$ and $\boldsymbol{P}_{\boldsymbol{E} \times \boldsymbol{B}}^{N}$ are defined in (71) and (72), respectively. Then, $\boldsymbol{P}^{\text {tot }, N}(t)$ satisfies the ordinary differential equation

$$
\frac{d}{d t}\left(\boldsymbol{P}_{f}^{N}(t)+\boldsymbol{P}_{\boldsymbol{E} \times \boldsymbol{B}}^{N}(t)\right)=\boldsymbol{R}(t),
$$


where the residual term on the right-hand side is given by

$$
\boldsymbol{R}(t)=-\frac{\omega_{c e}}{\omega_{p e}} \sum_{I} \int_{I} \mathcal{G}_{I}(\boldsymbol{x}, t) d \boldsymbol{x}-\sum_{I} \int_{\partial I}\left(\overline{\boldsymbol{n} \cdot \boldsymbol{g}_{\boldsymbol{v}}^{N}(\boldsymbol{x}, t)}+\mathcal{B}_{I}(\boldsymbol{x}, t)\right) d S
$$

Here, $\widehat{\boldsymbol{n} \cdot \boldsymbol{g}_{\boldsymbol{v}}^{N}}$ is the numerical flux associated with

$$
\boldsymbol{n} \cdot \boldsymbol{g}_{\boldsymbol{v}}^{N}(\boldsymbol{x}, t)=\sum_{s} m^{s} \int_{\Omega_{v}}(\boldsymbol{n} \cdot \boldsymbol{v}) \boldsymbol{v} f^{s, N}(\boldsymbol{x}, \boldsymbol{v}, t) d \boldsymbol{v},
$$

and

$$
\begin{aligned}
& \mathcal{G}_{I}(\boldsymbol{x}, t)=\frac{\omega_{c e}}{\omega_{p e}}\left(\boldsymbol{B}^{N} \times\left(\nabla_{\boldsymbol{x}} \times \boldsymbol{B}^{N}\right)+\boldsymbol{E}^{N} \times\left(\nabla_{\boldsymbol{x}} \times \boldsymbol{E}^{N}\right)\right)-\rho^{N} \boldsymbol{E}^{N}, \\
& \mathcal{B}_{I}(\boldsymbol{x}, t)=\left(\frac{\omega_{c e}}{\omega_{p e}}\right)^{2}\left(\widehat{\mathbb{F}_{\boldsymbol{E}}(\boldsymbol{U}) \boldsymbol{n}} \times \boldsymbol{B}^{N}-\widehat{\mathbb{F}_{\boldsymbol{B}}(\boldsymbol{U}) \boldsymbol{n}} \times \boldsymbol{E}^{N}\right),
\end{aligned}
$$

are a bulk and an elemental boundary term that depends on the approximate electromagnetic fields $\boldsymbol{E}^{N}, \boldsymbol{B}^{N}$, and $\rho^{N}$, and $\widehat{\mathbb{F}_{\boldsymbol{E}}(\boldsymbol{U}) \boldsymbol{n}}$ and $\widehat{\mathbb{F}_{\boldsymbol{B}}(\boldsymbol{U}) \boldsymbol{n}}$ are the numerical fluxes of $\mathbb{F}_{\boldsymbol{E}}(\boldsymbol{U}) \boldsymbol{n}$ and $\mathbb{F}_{\boldsymbol{B}}(\boldsymbol{U}) \boldsymbol{n}$ defined in (53).

We refer to Appendix B.2 for the proof of the theorem. Here, we further elaborate the result to investigate the meaning of the residual term. We consider the following formula from differential calculus: for a sufficiently smooth vector field $\boldsymbol{\zeta}$, it holds $\boldsymbol{\zeta} \times(\nabla \times \boldsymbol{\zeta})=\frac{1}{2} \nabla|\boldsymbol{\zeta}|^{2}-\nabla \cdot(\boldsymbol{\zeta} \otimes \boldsymbol{\zeta})+\boldsymbol{\zeta} \nabla \cdot \boldsymbol{\zeta}$. Hence,

$$
\begin{gathered}
\frac{\omega_{p e}}{\omega_{c e}} \mathcal{G}_{I}=\frac{1}{2} \nabla\left(\left|\boldsymbol{E}^{N}\right|^{2}+\left|\boldsymbol{B}^{N}\right|^{2}\right)-\nabla \cdot\left(\boldsymbol{E}^{N} \otimes \boldsymbol{E}^{N}+\boldsymbol{B}^{N} \otimes \boldsymbol{B}^{N}\right)+\boldsymbol{E}^{N} \nabla \cdot \boldsymbol{E}^{N}+\boldsymbol{B}^{N} \nabla \cdot \boldsymbol{B}^{N}-\frac{\omega_{p e}}{\omega_{c e}} \rho^{N} \boldsymbol{E}^{N} \\
=\mathcal{H}_{I}+\boldsymbol{E}^{N}\left(\nabla \cdot \boldsymbol{E}^{N}-\frac{\omega_{p e}}{\omega_{c e}} \rho^{N}\right)+\boldsymbol{B}^{N} \nabla \cdot \boldsymbol{B}^{N},
\end{gathered}
$$

with

$$
\mathcal{H}_{I}=\frac{1}{2} \nabla\left(\left|\boldsymbol{E}^{N}\right|^{2}+\left|\boldsymbol{B}^{N}\right|^{2}\right)-\nabla \cdot\left(\boldsymbol{E}^{N} \otimes \boldsymbol{E}^{N}+\boldsymbol{B}^{N} \otimes \boldsymbol{B}^{N}\right) .
$$

We sum term $\mathcal{H}_{I}$ over all the cells $I$, use the Gauss-Green formula, and rearrange the summation over the faces using the periodicity in space, to get

$$
\begin{aligned}
\sum_{I} \int_{I} \mathcal{H}_{I} d \boldsymbol{x} & =\sum_{I} \int_{\partial I}\left(\frac{1}{2} \boldsymbol{n}\left(\left|\boldsymbol{E}^{N}\right|^{2}+\left|\boldsymbol{B}^{N}\right|^{2}\right)-\boldsymbol{n} \cdot\left(\boldsymbol{E}^{N} \otimes \boldsymbol{E}^{N}+\boldsymbol{B}^{N} \otimes \boldsymbol{B}^{N}\right)\right) d \boldsymbol{x} \\
& =\frac{1}{2} \sum_{\mathrm{f}} \int_{\mathrm{f}}\left(\llbracket\left|\boldsymbol{E}^{N}\right|^{2} \rrbracket_{\mathrm{f}}+\llbracket\left|\boldsymbol{B}^{N}\right|^{2} \rrbracket_{\mathrm{f}}\right) d S-\sum_{\mathrm{f}} \int_{\mathrm{f}}\left(\llbracket \boldsymbol{E}^{N} \otimes \boldsymbol{E}^{N} \rrbracket_{\mathrm{f}}+\llbracket \boldsymbol{B}^{N} \otimes \boldsymbol{B}^{N} \rrbracket_{\mathrm{f}}\right) \cdot \boldsymbol{n}_{\mathrm{f}} d S,
\end{aligned}
$$

where in the last equation we set $\boldsymbol{n}_{\mathrm{f}}=\boldsymbol{n}_{\mathrm{f}}^{+}$, and the jumps of $\boldsymbol{E}^{N} \otimes \boldsymbol{E}^{N}$ and $\boldsymbol{B}^{N} \otimes \boldsymbol{B}^{N}$ are defined as

$$
\begin{gathered}
\llbracket \boldsymbol{E}^{N} \otimes \boldsymbol{E}^{N} \rrbracket_{\mathrm{f}}=\boldsymbol{n}^{+} \cdot\left(\boldsymbol{E}^{N} \otimes \boldsymbol{E}^{N}\right)^{+} \cdot \boldsymbol{n}^{+}+\boldsymbol{n}^{-} \cdot\left(\boldsymbol{E}^{N} \otimes \boldsymbol{E}^{N}\right)^{-} \cdot \boldsymbol{n}^{-}, \\
\llbracket \boldsymbol{B}^{N} \otimes \boldsymbol{B}^{N} \rrbracket_{\mathrm{f}}=\boldsymbol{n}^{+} \cdot\left(\boldsymbol{B}^{N} \otimes \boldsymbol{B}^{N}\right)^{+} \cdot \boldsymbol{n}^{+}+\boldsymbol{n}^{-} \cdot\left(\boldsymbol{B}^{N} \otimes \boldsymbol{B}^{N}\right)^{-} \cdot \boldsymbol{n}^{-},
\end{gathered}
$$

with the interpretation that $\boldsymbol{n}^{+} \cdot\left(\boldsymbol{E}^{N} \otimes \boldsymbol{E}^{N}\right)^{+} \cdot \boldsymbol{n}^{+}=\left(\boldsymbol{n}^{+}\right)^{T}\left(\boldsymbol{E}^{N}\left(\boldsymbol{E}^{N}\right)^{T}\right)^{+} \boldsymbol{n}^{+}$, and similarly for the other terms.

We can analogously transform the integral term involving $\mathcal{B}_{I}$ by rearranging the summation of the elemental boundary integrals as summation of elemental interface integrals 


$$
\begin{aligned}
\sum_{I} \int_{\partial I} \mathcal{B}_{I} d S & =\left(\frac{\omega_{c e}}{\omega_{p e}}\right)^{2} \sum_{I} \int_{\partial I}\left(\widehat{\mathbb{F}_{\boldsymbol{E}}(\boldsymbol{U}) \boldsymbol{n}} \times \boldsymbol{B}^{N}+\widehat{\mathbb{F}_{\boldsymbol{B}}(\boldsymbol{U}) \boldsymbol{n}} \times \boldsymbol{E}^{N}\right) d S \\
& =\sum_{\mathrm{f}} \int_{\mathrm{f}}\left(\widetilde{\llbracket \mathbb{F}_{\boldsymbol{E}}(\boldsymbol{U}) \boldsymbol{n}_{\mathrm{f}}} \times \boldsymbol{B}^{N} \rrbracket+\llbracket \widetilde{\mathbb{F}_{\boldsymbol{B}}(\boldsymbol{U}) \boldsymbol{n}_{\mathrm{f}}} \times \boldsymbol{E}^{N} \rrbracket\right) d S,
\end{aligned}
$$

with the jumps defined as

$$
\begin{aligned}
& \llbracket \widehat{\llbracket \mathbb{F}_{\boldsymbol{E}}(\boldsymbol{U}) \boldsymbol{n}} \times \boldsymbol{B}^{N} \rrbracket_{f}:=\widehat{\mathbb{F}_{\boldsymbol{E}}\left(\boldsymbol{U}^{-}\right) \boldsymbol{n}^{+}} \times\left(\boldsymbol{B}^{N}\right)^{-}+\widehat{\mathbb{F}_{\boldsymbol{E}}\left(\boldsymbol{U}^{+}\right) \boldsymbol{n}^{-}} \times\left(\boldsymbol{B}^{N}\right)^{+}, \\
& \llbracket \widetilde{\mathbb{F}_{\boldsymbol{B}}(\boldsymbol{U}) \boldsymbol{n}} \times \boldsymbol{E}^{N} \rrbracket_{f}:=\widehat{\mathbb{F}_{\boldsymbol{B}}\left(\boldsymbol{U}^{-}\right) \boldsymbol{n}^{+}} \times\left(\boldsymbol{E}^{N}\right)^{-}-\widehat{\mathbb{F}_{\boldsymbol{B}}\left(\boldsymbol{U}^{+}\right) \boldsymbol{n}^{-}} \times\left(\boldsymbol{E}^{N}\right)^{+} .
\end{aligned}
$$

Since the numerical fluxes $\widehat{\mathbb{F}_{\boldsymbol{E}}(\boldsymbol{U}) \boldsymbol{n}}$ and $\widehat{\mathbb{F}_{\boldsymbol{B}}(\boldsymbol{U}) \boldsymbol{n}}$ depend on $\boldsymbol{B}^{N}$ and $\boldsymbol{E}^{N}$, respectively, the integral term involving $\mathcal{B}_{I}$ also depends on the jumps of the electromagnetic fields, and it is quadratic on $\boldsymbol{B}^{N}$ and $\boldsymbol{E}^{N}$.

These developments show that if the exact solutions $f^{s}, \boldsymbol{E}$ and $\boldsymbol{B}$ are sufficiently regular (at least continuous) the residual term must tend to zero at least at the convergence rate determined by the order of the approximation. Indeed, $\mathcal{H}_{I}$ depends on the square of the jumps of the electromagnetics fields, which must tend to zero if $\boldsymbol{E}^{N}$ and $\boldsymbol{B}^{N}$ are approximations of continuous functions. The last two terms in (78) must tend to zero as $\mathcal{O}\left(h^{N_{D G}}\right)$, where $h=\max (\Delta x, \Delta y, \Delta z)$. Similar considerations hold for the term $\mathcal{B}_{I}$.

\subsection{Conservation properties of the total energy}

The total energy of the plasma is defined as $\mathcal{E}(t)=\mathcal{E}_{\text {kin }}(t)+\mathcal{E}_{\boldsymbol{E}, \boldsymbol{B}}(t)$, where

$$
\begin{aligned}
\mathcal{E}_{\text {kin }}(t) & :=\frac{1}{2} \sum_{s} m^{s} \sum_{I} \int_{I}\left(\int_{\Omega_{v}}|\boldsymbol{v}|^{2} f^{s}(\boldsymbol{x}, \boldsymbol{v}, t) d \boldsymbol{v}\right) d \boldsymbol{x}, \\
\mathcal{E}_{\boldsymbol{E}, \boldsymbol{B}}(t) & :=\frac{1}{2}\left(\frac{\omega_{c e}}{\omega_{p e}}\right)^{2} \int_{\Omega_{x}}\left(|\boldsymbol{E}(\boldsymbol{x}, t)|^{2}+|\boldsymbol{B}(\boldsymbol{x}, t)|^{2}\right) d \boldsymbol{x} .
\end{aligned}
$$

Let $\left|\mathbb{F}_{\beta}\right|=\mathbb{F}_{\beta}^{+}-\mathbb{F}_{\beta}^{-}, \beta \in\{x, y, z\}$, and $|\mathbb{F}|=\left|\mathbb{F}_{x}\right|+\left|\mathbb{F}_{y}\right|+\left|\mathbb{F}_{z}\right|$. For a generic mesh face $\boldsymbol{f}$ we introduce the quantity

$$
\mathcal{J}_{\mathfrak{f}}(t):= \begin{cases}0 & \text { for central numerical flux, } \\ \frac{1}{2} \int_{\mathrm{f}} \llbracket \boldsymbol{U}(t) \rrbracket_{\mathrm{f}} \cdot|\mathbb{F}| \cdot \llbracket \boldsymbol{U}(t) \rrbracket_{\mathrm{f}} d S & \text { for upwind numerical flux }\end{cases}
$$

where

$$
\llbracket \boldsymbol{U}(t) \rrbracket_{\mathrm{f}}=\boldsymbol{n}^{+} \cdot \boldsymbol{U}\left(\boldsymbol{x}_{\mathrm{f}}^{+}, t\right)+\boldsymbol{n}^{-} \cdot \boldsymbol{U}\left(\boldsymbol{x}_{\mathrm{f}}^{-}, t\right)=: \boldsymbol{n}^{+} \cdot \boldsymbol{U}_{\mathrm{f}}^{+}(t)+\boldsymbol{n}^{-} \cdot \boldsymbol{U}_{\mathrm{f}}^{-}(t),
$$

is the jump of $\boldsymbol{U}$ across the mesh face f, and $\boldsymbol{U}_{\mathrm{f}}^{ \pm}(t)=\boldsymbol{U}\left(\boldsymbol{x}^{ \pm}, t\right)$ are shortcuts to denote the trace of $\boldsymbol{U}$ on the opposite sides of $f$.

The following result shows that the total discrete energy is exactly conserved when central numerical fluxes (64) are employed in the approximation of Maxwell's equations, while a numerical dissipation proportional to the jump of the approximate electromagnetic fields occurs when upwind numerical fluxes (65) are used. We refer to Appendix B.3 for the complete proof of the statement.

Theorem 5.3 (Conservation of the total energy) Let $\left(f^{s, N}(\boldsymbol{x}, \boldsymbol{v}, t), \boldsymbol{E}^{N}(\boldsymbol{x}, t), \boldsymbol{B}^{N}(\boldsymbol{x}, t)\right)$ be the numerical solution of the semi-discrete Vlasov-Maxwell problem with periodic boundary conditions in space. Let the total discrete energy of the plasma be defined as

$$
\begin{aligned}
\mathcal{E}^{N}(t) & =\frac{1}{2} \sum_{s} m^{s} \sum_{I} \int_{I}\left(\int_{\Omega_{v}}|\boldsymbol{v}|^{2} f^{s, N}(\boldsymbol{x}, \boldsymbol{v}, t) d \boldsymbol{v}\right) d \boldsymbol{x}+\frac{1}{2}\left(\frac{\omega_{c e}}{\omega_{p e}}\right)^{2} \int_{\Omega_{x}}\left(\left|\boldsymbol{E}^{N}(\boldsymbol{x}, t)\right|^{2}+\left|\boldsymbol{B}^{N}(\boldsymbol{x}, t)\right|^{2}\right) d \boldsymbol{x} \\
& =: \mathcal{E}_{\mathbf{k i n}}^{N}(t)+\mathcal{E}_{\boldsymbol{E}, \boldsymbol{B}}^{N}(t) .
\end{aligned}
$$


Then, the variation in time of the total discrete energy satisfies

$$
\frac{d \mathcal{E}^{N}(t)}{d t}=-\left(\frac{\omega_{c e}}{\omega_{p e}}\right)^{2} \sum_{\mathrm{f}} \mathcal{J}_{\mathrm{f}}(t) \leq 0
$$

\section{Time discretization}

The semi-discrete Hermite-DG scheme of the Vlasov equation on a local element $I$ for a fixed Hermite triplet $(n, m, p)$, and $l$-th Legendre polynomial reads

$$
\mu_{I, l} \frac{d C_{n, m, p}^{s, I, l}(t)}{d t}+\sum_{\beta \in\{x, y, z\}}\left(-\left[\mathcal{I}^{\beta, s}\right]_{n, m, p}+\left(\delta_{\beta}^{s,+}-\delta_{\beta}^{s,-}\right)\right)-\sum_{\beta \in\{x, y, z\}}\left(\left[\mathcal{I}^{E_{\beta}}\right]_{n, m, p}+\left[\mathcal{I}^{B_{\beta}}\right]_{n, m, p}\right)=0,
$$

where the $\left\{\mathcal{I}^{\beta, s}\right\}_{\beta}$ are defined in (35a), (35b), (35c), $\left\{\mathcal{I}^{E_{\beta}}\right\}_{\beta}$ and $\left\{\mathcal{I}^{B_{\beta}}\right\}_{\beta}$ are defined in (44), (45), and $\left\{\delta_{\beta}^{s, \pm}\right\}_{\beta}$ have been introduced in (37). The semi-discrete DG scheme for the Maxwell wave propagation equation is as in (67), namely

$$
\mu_{I, l} \frac{d \boldsymbol{U}^{I, l}(t)}{d t}-\mathcal{I}_{F}+\int_{\partial I} \widehat{\mathbb{F}(\boldsymbol{U}) \boldsymbol{n}} \varphi^{I, l} d S=\int_{I} \boldsymbol{W}^{N}(\boldsymbol{U}) \varphi^{I, l} d \boldsymbol{x} .
$$

Each term is defined in Section 4.

The time discretization we propose is based on explicit adaptive Runge-Kutta (RK) methods in the framework of the method of lines. In particular, for the numerical experiments reported in Section 8, we considered three Runge-Kutta algorithms: (i) the non-adaptive third-order accurate RK scheme of BogackiShampine, cf. [13]; (ii) the adaptive third-order accurate RK scheme of Bogacki-Shampine with second order embedded method, cf. [13]; (iii) the non-adaptive fifth-order accurate Fehlberg RK scheme, cf. [43].

Finally, we note that all explicit (and implicit) RK methods conserve linear invariants, see, for example, [49, Theorem 1.5]; hence, we can extend the result of Theorem 5.1 to the fully discrete case and conclude that the total number of particles is conserved. Instead, [49, Theorem 2.2] shows that quadratic invariants can be conserved by explicit RK methods only under an algebraic condition on the coefficients of the scheme. Consequently, we do not expect that a result corresponding to Theorem 5.3 may exist in the fully discrete setting for our RK methods, implying that the total energy in the system is not conserved. The total momentum is also not expected to be conserved in the fully discrete case, since it is already not an invariant according to Theorem 5.2 in the semi-discrete case. However, from theoretical considerations (see, again, [59]), we can expect that the violation in the conservation of total momentum and energy are small and decreasing at least at the rate of convergence of the numerical approximation.

\subsection{Artificial collisional operator}

Collisionless plasmas can develop finer and finer scales in velocity space, a phenomenon known as filamentation. This can lead to recurrence once velocity-space structures reach scales that are no longer resolved by the particular numerical algorithm considered [23, 25, 54]. It is therefore customary to introduce an artificial collisional operator to damp high order modes and prevent filamentation. We adopt here the same collisional operator introduced in Ref. [38],

$$
\begin{aligned}
\mathcal{C}_{n, m, p}\left[C^{s}\right]=-\nu & {\left[\frac{n(n-1)(n-2)}{N_{v_{x}}\left(N_{v_{x}}-1\right)\left(N_{v_{x}}-2\right)}+\frac{m(m-1)(m-2)}{N_{v_{y}}\left(N_{v_{y}}-1\right)\left(N_{v_{y}}-2\right)}\right.} \\
& \left.+\frac{p(p-1)(p-2)}{N_{v_{z}}\left(N_{v_{z}}-1\right)\left(N_{v_{z}}-2\right)}\right] C_{n, m, p}^{s}(\boldsymbol{x}, t),
\end{aligned}
$$

where $\nu$ is the collision rate. The operator in Eq. (87) is added to the right hand side of the Vlasov equation (20) (Eq. (85) in semi-discrete form) for each Hermite mode. This type of collisional operator has the 
advantage that it does not act directly on the first three Hermite modes and therefore does not affect the conservation laws for total mass, momentum and energy presented in Section 5 (see also Ref. [38]). Obviously, the collisional operator must always be used in a convergence sense, i.e. making sure that it does not affect the collisionless physics of interest significantly.

\subsection{Numerical Stability}

Previous investigations of the methods based on the Hermite expansion found no stability theorem for the asymmetrically-weighted Hermite representation, i.e. the $L_{2}$ of the distribution function is not bounded and can grow in time [e.g. 78]. In contrast, the symmetrically-weighted expansion does conserve the $L_{2}$ norm and is therefore numerically stable. In practice, the absence of the stability theorem does not mean that the simulations necessarily blow up in time. In our experiments, the instability clearly manifests itself only in situations where a physical quantity significant for the dynamics is not properly resolved. For these cases, when the resolution is increased, the instability typically goes away. In particular, in the results presented in the paper, we have seen no sign of numerical instabilities. We note that while the collisional operator discussed above does provide additional stabilizing influence, its primary role is to control filamentation and recurrence in a manner which is common to all methods attempting to solve the Vlasov equation using a limited number of degrees of freedom.

\section{Implementation details and parallel scalability}

The implementation of the Hermite-DG discretization that we designed in the previous sections refers specifically to Eqs. (85)-(86). The implementation is carried out in the framework of the Spectral Plasma Solver (SPS), a computational software currently under development at the Los Alamos National Laboratory for the numerical modeling of multiscale phenomena in collisionless or weakly collisional plasmas. This software incorporates a spectral-based solver for the Vlasov equations and the coupled electromagnetic models, which is an implementation of the numerical methods described in References $[20,38,84,85]$. The implementation of the algorithms discussed in this paper is a sub-branch of SPS, and, for brevity, it will be referred to as $S P S-D G$.

The SPS-DG code is based on the method of lines. The numerical discretization is expressed by the system of ordinary differential equations (ODEs)

$$
\frac{d \boldsymbol{W}}{d t}=\boldsymbol{G}(\boldsymbol{W})
$$

where $\boldsymbol{W}(t)$ is the state vector collecting the time-dependent coefficients $C_{n, m, p}^{s, l, l}(t)$ and $\boldsymbol{U}^{I, l}(t)$ resulting from the Hermite-DG expansion of the species distribution functions $f^{s}$ and the electromagnetic fields $\boldsymbol{E}$ and $\boldsymbol{B} ; \boldsymbol{G}$ is the vector-valued function containing all information about the phase space discretization.

In this section, we discuss three aspects of the design and implementation of SPS-DG: (i) the data structure storing the global state vector $\boldsymbol{W}$; (ii) the action of function $\boldsymbol{G}$; (iii) the temporal discretization. Finally, we discuss the parallel scalability of the SPS-DG code and we show by a numerical experiment that the Hermite-DG approach leads to efficient implementations on high-performance-computing architectures.

\subsection{Data structure of the global state vector and parallelization}

The data structure used to store the global state vector $\boldsymbol{W}$ was chosen to achieve efficient parallelization of the code. The parallel version of SPS-DG is designed to run on distributed memory architectures and is currently based on the domain decomposition of the physical space while the velocity space is not decomposed. This choice guarantees high locality of the data structures of the code, and hence, high parallel efficiency as demonstrated by the scalability study shown at the end of this section. 
The parallel implementation of the domain decomposition in SPS-DG relies on the Portable, Extensible Toolkit for Scientific Computation (PETSc) developed at Argonne National Laboratory for the scalable (parallel) solution of scientific applications modeled by partial differential equations. Parallelism in PETSc is achieved through the Message Passing Interface (MPI) standard for all message-passing communications.

We exploit DMDA, which is the native PETSc parallel data structure for structured grids, to accommodate the DG cells $I$, see PETSc user manual [7]. DMDA is a PETSc object that manages an abstract grid object and its interactions with the algebraic solvers. The other degrees of freedom located within the DG cells are internally stored in each DMDA point.

The total number of degrees of freedom (DOFs) that we need to store to represent the state vector $\boldsymbol{W}$ at each time instant is

$$
\# \text { DOFs }=N_{x} N_{y} N_{z} N_{l}\left(6+N_{s}\left(N_{v_{x}}+1\right)\left(N_{v_{y}}+1\right)\left(N_{v_{z}}+1\right)\right),
$$

where $N_{s}$ is the number of different plasma species. For example, in the Orszag-Tang vortex test that we consider below for the scalability study and in the section of the numerical experiments, we consider $N_{s}=2$, $N_{x} \sim N_{y} \sim N_{z} \sim 100, N_{l} \sim N_{v_{x}} \sim N_{v_{y}} \sim N_{v_{z}} \sim 10$, and total number of DOFs may be in the range from $\sim 0.02$ up to 1 trillions. Modern supercomputers can perform kinetic plasma simulations with trillions of DOFs [14].

Since the space decomposition is performed along each spatial dimension, i.e., along $x, y$ and $z$, the maximum number of MPI processes that SPS-DG can use is $N_{x} N_{y} N_{z}$, which is of the order of $10^{6}-10^{7}$ even for the smallest 3D simulations, ensuring that a high degree of parallelism is available. At the same time, partitioning only the spatial directions reduces the amount of required communications between processes, thus improving the parallelization efficiency, since the DOFs pertained to a selected DG cell always reside on the same processor. Moreover, the communication is only needed to compute the numerical flux from adjacent DG cells, which requires to transfer information only between processors with high affinity.

The DMDA data structure is also responsible for managing the boundary conditions. The SPS-DG code can incorporate two distinct types of boundary conditions: (i) periodic boundary conditions, which are automatically handled by DMDA; and (ii) ghost cells, where the user is responsible for providing the state vector $\boldsymbol{W}$ on the boundary at each time step.

\subsection{Phase space discretization}

The procedure to evaluate the function $\boldsymbol{G}$ at each time step is determined by the Hermite-DG discretization of the phase space that we detailed in Sections 3 and 4. The SPS-DG code does not perform any numerical integration to evaluate $\boldsymbol{G}$. Indeed, all integrals in the system (85)-(86) are computed analytically by using the orthogonality relations and exact formulas (24a)-(24c). Additionally, all linear matrices in the definition of the upwind numerical flux in the discretization of the Vlasov and Maxwell equations, i.e., $\mathbb{A}_{\beta}, \mathbb{F}_{\beta}$ and their upwind decompositions $\mathbb{A}_{\beta}^{ \pm}, \mathbb{F}_{\beta}^{ \pm}$, are precomputed and stored at the beginning of every simulation, so they are never computed during the evaluation of $\boldsymbol{G}$.

\subsection{Implementation of the time-stepping scheme}

The implementation of the SPS-DG code uses the external time-stepping library TS of the PETCs framework, cf. $[7,8,9]$, which provides optimized and thoroughly benchmarked implementations of explicit RungeKutta methods.

\subsection{Scalability}

SPS-DG achieves good parallel scalability on different high-performance-computing platforms, including the clusters available at the Los Alamos National Laboratory (LANL). An example of the scalability of SPS-DG is shown in Figure 2, which reports the elapsed times versus the number of cores in the numerical 


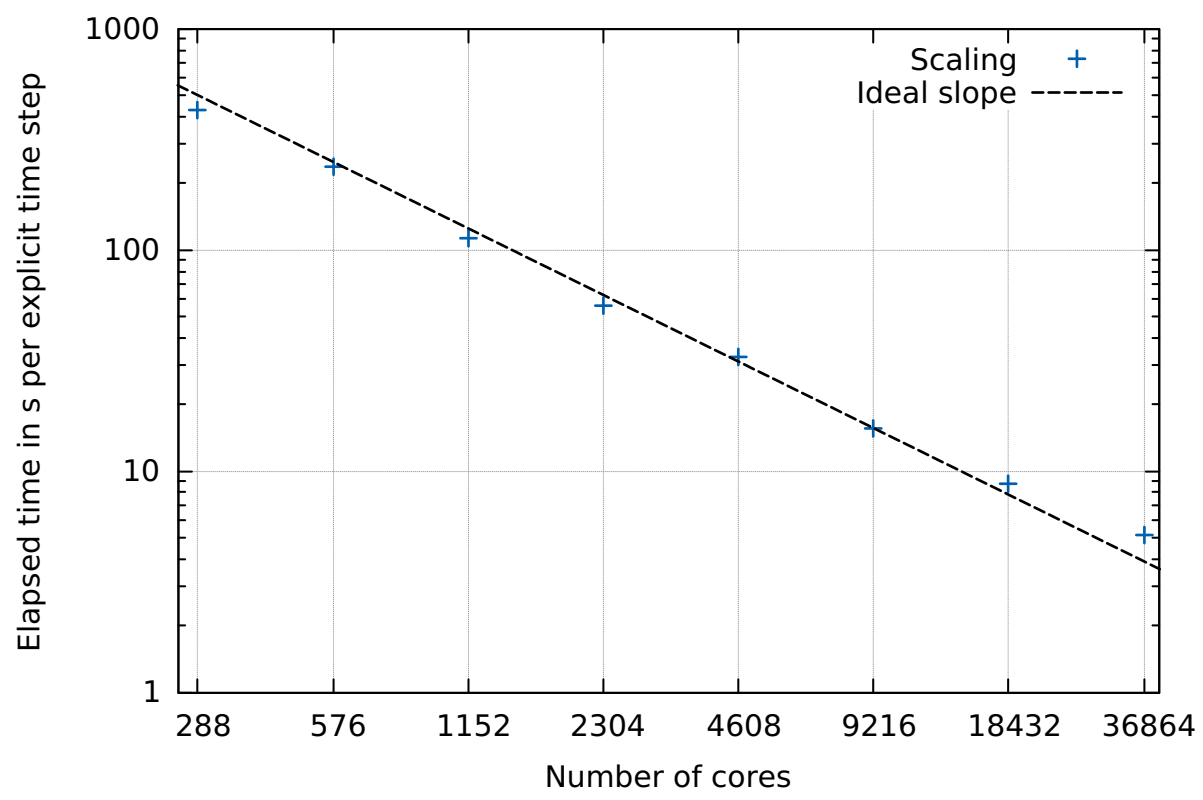

Fig. 2. Strong scalability test for Orszag-Tang problem. The test was performed on LANL's cluster Grizzly (CTS-1 cluster with 8-SU, 1490 nodes, 53640 cores, 191 TB memory, 1.8 Pflops peak operating speed).

simulation of the (2D-3V) magnetized plasma turbulence decay problem, known as Orszag-Tang vortex test. More details about this test case and the performance of the SPS-DG code will be given in Section 8.4. We considered $N_{x}=N_{y}=768, N_{z}=1\left(L_{x}=L_{y}=200, L_{z}=1\right)$ spatial grid cells and a maximum local polynomial degree $N_{D G}=2$ in the DG approximation, and, for the velocity resolution, $N_{v_{x}}=N_{v_{y}}=N_{v_{z}}=9$ Hermite modes. The total number of degrees of freedom per DG cell is 12036 corresponding to $\sim 7$ billions of DOFs in total. Time stepping was provided by the third order RK integrator in the TS library with constant time step $\Delta t=0.05$.

The code was run for 20 time steps on the LANL cluster Grizzly and the elapsed time at the end of each run was normalized to that for one time step. Grizzly is a Commodity Technology System, version 1 with 8 Scalable Units (CTS-1 cluster with 8-SU) and 18 additional compute nodes. In total, Grizzly has 1490 and 53640 compute nodes and cores, respectively, with a total of 191TB cluster memory. Grizzly peak operating speed is 1.8 Pflops. Figure 2 shows nearly ideal scalability for a broad range of core numbers. Perfect scalability is usually achieved when the number of degrees of freedom is larger or comparable to $10^{5}$ (we recall that the absolute minimum number of DOFs recommended by PETSc developers is $10^{4}[6]$ ), even if the number of DG cells per core is small, i.e., $\sim 1$ (in the case of 36864 cores, this test has $1.9 \cdot 10^{5}$ degrees of freedom per cell and 16 DG cells per core).

\section{Numerical results}

In this section we perform tests to benchmark and assess the accuracy of the SPS-DG framework.

\subsection{Accuracy test for spatial discretization}

We start with a manufactured solution test to assess the accuracy of the spatial discretization in our implementation. Instead of using a known manufactured solution that prescribes a source term, we exploit the time reversibility of the Vlasov-Maxwell system. Indeed, we can integrate the Vlasov-Maxwell system forward and backward in time and return to the initial state, possibly modified by the numerical integration errors. Equivalently, we integrate the Vlasov-Maxwell system forward in time from $t=0$ up to $t=T$ for 
a given final time $T>0$. Then, we reverse the velocity coordinate and the direction of the magnetic field (recall that $\boldsymbol{B}$ is a pseudovector), as follows

$$
f^{s}(\boldsymbol{x}, \boldsymbol{v}, t) \longrightarrow f^{s}(\boldsymbol{x},-\boldsymbol{v}, t), \quad \mathbf{B} \longrightarrow-\mathbf{B},
$$

and continue the integration from $t=T$ to $t=2 T$. Since the Hermite expansion of the Vlasov equation does not break the time reversibility, we can use this test to assess the numerical errors that are introduced by the discontinuous Galerkin discretization in space. To this end, we need a sufficiently small time step $\Delta t$ and a RK time-stepping method that is sufficiently accurate to ensure that the time discretization error is much smaller than the spatial discretization error.

In this test, we discretize the velocity space by setting $N_{v_{x}}=N_{v_{y}}=N_{v_{z}}=3$, so to have 4 polynomials in each velocity direction, and the physical space domain $\Omega_{x}=\left[0, L_{x}\right] \times\left[0, L_{y}\right] \times\left[0, L_{z}\right]$ by setting $L_{x}=$ $L_{y}=L_{z}=1$ and $N_{x}=N_{y}=N_{z}=12,24,48,96,192,384$. The cell size is uniform in every direction. Periodic boundary conditions are assumed. The degree of the local discontinuous polynomials is set to $N_{D G}=0,1,2,3$. Note that the case $N_{D G}=0$ corresponds to a low-order finite volume approximation.

We initialize the magnetic field $B_{x}=1$ and the electron-proton plasma with Maxwellian distributions

$$
f^{s}(\boldsymbol{x}, \boldsymbol{v}, 0)=\prod_{\beta \in\{x, y, z\}} \frac{1}{v_{T_{\beta}^{s}} \sqrt{2 \pi}} \exp \left[-\frac{v_{\beta}^{2}}{2 v_{T_{\beta}^{s}}^{2}}\right],
$$

where $s \in\{e, i\}$ (respectively, electrons or ions), $v_{T_{\beta}^{s}}$ is the thermal velocity along direction $\beta \in\{x, y, z\}$ for species $s$. The Maxwellian distribution corresponds to only one term in the asymmetrically weighted Hermite polynomial expansion (cf. (69)), namely

$$
C_{0,0,0}^{s}(\boldsymbol{x})=\frac{1}{\alpha_{x}^{s} \alpha_{y}^{s} \alpha_{z}^{s}},
$$

where $\alpha_{\beta}^{s}=\sqrt{2} v_{T_{\beta}^{s}}$ and $u_{\beta}^{s}=0$ for $\beta \in\{x, y, z\}$. We use realistic ion-to-electron mass ratio $m_{i} / m_{e}=1836$, thermal velocities $v_{T_{\beta}^{e}}=v_{T_{\beta}^{i}} \sqrt{m_{i} / m_{e}}=0.125$ for $\beta \in\{x, y, z\}$, and electron plasma frequency/gyrofrequency ratio $\omega_{p e} / \omega_{c e}=4$. We also introduce a current perturbation $J_{z}(\boldsymbol{x})=\sin (2 \pi x+1) \sin (2 \pi y+2) \sin (2 \pi z+3)$ by exciting $C_{0,0,1}^{e}$, cf. (68). The other coefficients in the Hermite expansion are initialized to zero.

In order to avoid pollution of the results by time discretization errors, we integrate the system forward up to time $T=1$ and then backward for an equivalent time interval with highly accurate fifth-order RK method [43] with time step $\Delta t=10^{-3}$ (two thousand explicit time steps in total). The resulting $L^{2}$ errors for the electron distribution function, electric and magnetic fields versus number of total degrees of freedom are shown in Figure 3 for the central numerical flux and in Figure 4 for the upwind numerical flux in the Maxwell equations. We do not compute the error for the ion distribution function because ions are practically motionless on such short electron time scales.

In every figure in this subsection, we measure the order of convergence of the method by fitting data from the last two (most resolved) points. The computed orders for different $N_{D G}$ and numerical fluxes are summarized in Table 1. For cases with the central numerical flux, we can see that order of convergence measured numerically is in good agreement with the theoretical prediction, i.e., $N_{D G}+1$. In case of the upwind numerical flux, the order measured numerically for the electron distribution function error follows the same theoretical prediction. However, errors in electric and magnetic fields show a consistent reduction in the computed order, so that the electromagnetic field errors follow approximately an $N_{D G}+1 / 2$ order of convergence. This convergence rate is consistent with the study of Reference [27], where the DG discretization with the upwind numerical flux was applied to the Vlasov-Maxwell system. 
Table 1

\begin{tabular}{|l|c|c|c|c|}
\hline & $N_{D G}=0$ & $N_{D G}=1$ & $N_{D G}=2$ & $N_{D G}=3$ \\
\hline Electron distribution function with central flux & 0.96 & 2.02 & 3.00 & 4.00 \\
\hline Electric field with central flux & 0.97 & 1.99 & 2.99 & 3.98 \\
\hline Magnetic field with central flux & 0.97 & 2.36 & 2.87 & 3.97 \\
\hline Electron distribution function with upwind flux & 0.94 & 2.14 & 3.00 & 4.00 \\
\hline Electric field with upwind flux & 0.80 & 1.57 & 2.62 & 3.56 \\
\hline Magnetic field with upwind flux & 0.79 & 1.61 & 2.64 & 3.53 \\
\hline
\end{tabular}

Manufactured solution benchmark: numerical rate of convergence of the spatial discretization for different polynomial degrees $N_{D G}$ using central and upwind numerical fluxes to solve the Maxwell equations.
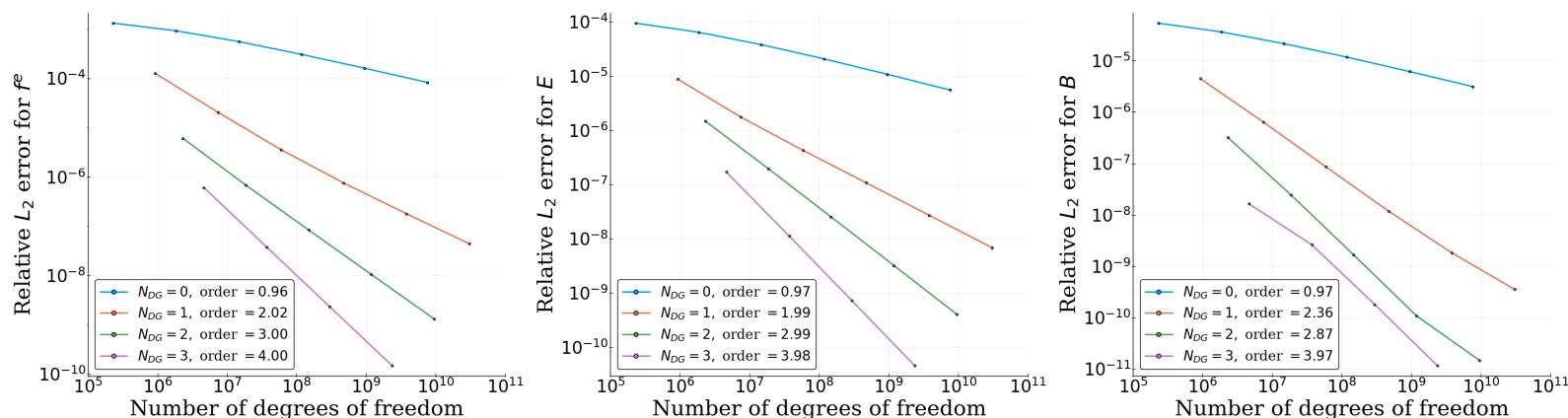

Fig. 3. Manufactured solution benchmark: $L_{2}$ errors in electron particle distribution function (left panel), electric field (central panel), and magnetic field (right panel) using the central numerical flux to solve the Maxwell equations.
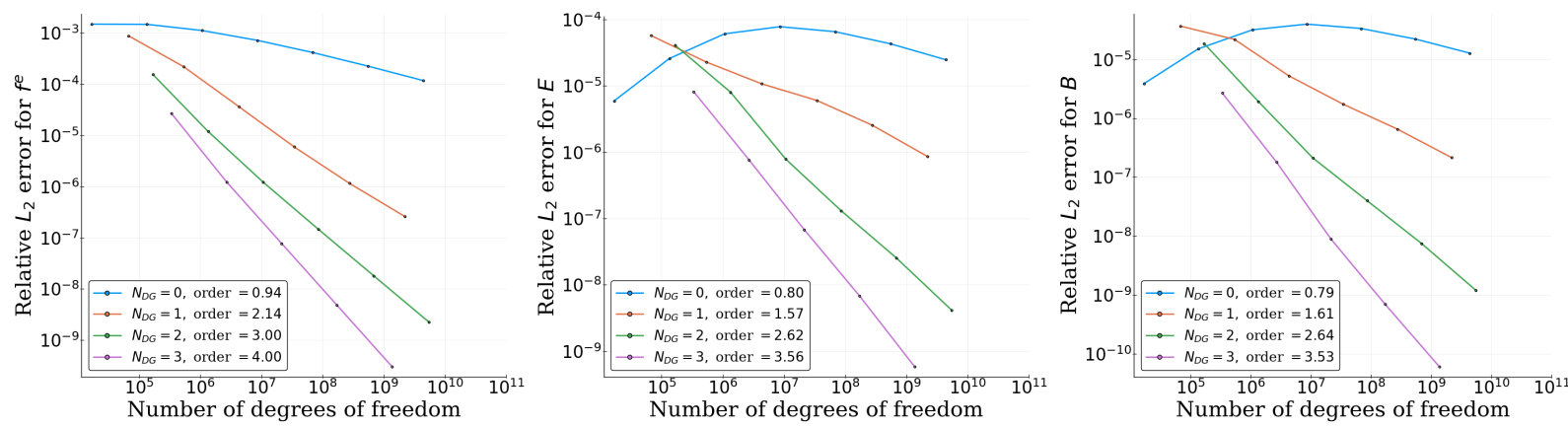

Fig. 4. Manufactured solution benchmark: $L_{2}$ errors in electron particle distribution function (left panel), electric field (central panel), and magnetic field (right pane) using the upwind numerical flux to solve the Maxwell equations.

\subsection{Whistler instability}

In the second benchmark problem we investigate the whistler instability. Since the instability is driven by the electron temperature anisotropy and cyclotron resonance, cf. [46], this test asserts the method's ability to describe kinetic physics. The whistler instability is common in space plasmas and it is believed to be behind the generation of chorus waves in the Earth's magnetosphere [82].

We discretize the velocity space by setting $N_{v_{x}}=N_{v_{y}}=N_{v_{z}}=9$, so to have 10 polynomials in each velocity direction, and the physical space domain $\Omega_{x}=\left[0, L_{x}\right] \times\left[0, L_{y}\right] \times\left[0, L_{z}\right]$ by setting $L_{x}=2 \pi$, $L_{y}=L_{z}=1$ and $N_{x}=50, N_{y}=N_{z}=1$, with uniform cell size in every direction.

Further, we consider $B_{x}=1$ for the initial magnetic field, while the distribution functions of electrons and ions (protons) are assumed to be Maxwellian. Therefore, we set

$$
C_{0,0,0}^{s}(\boldsymbol{x})=\frac{1}{\alpha_{x}^{s} \alpha_{y}^{s} \alpha_{z}^{s}}
$$


where $s \in\{e, i\}$ (electrons or ions), $\alpha_{\beta}^{s}=\sqrt{2} v_{T_{\beta}^{s}}$, and $u_{\beta}^{s}=0$ for $\beta \in\{x, y, z\}$. We use a realistic ionto-electron mass ratio $m_{i} / m_{e}=1836$, thermal velocities $v_{T_{\beta}^{e}}=v_{T_{\beta}^{i}} \sqrt{m_{i} / m_{e}}=0.125, \beta \in\{x, y, z\}$ with the exception of the reduced electron thermal velocity along the $x$ axis, $v_{T_{x}^{e}}=0.056$, to create anisotropy and, thus, the source of the instability. The electron plasma/gyrofrequency ratio is $\omega_{p e} / \omega_{c e}=4$, and the collisional operator has $\nu=1$. In order to seed the whistler instability, we initialize a small electron current perturbation along $x, j_{x}^{e}(\boldsymbol{x})=10^{-3} \cos (x)$, which can be imposed through the first Hermite moment $C_{1,0,0}^{e}$. The other coefficients in the Hermite expansion are initialized to zero.

To advance the numerical solution in time, we use the third order non-adaptive RK scheme of BogackiShampine [13] with time steps $\Delta t=0.02,0.01,0.005$. We also assess the performance of the method for the two alternative choices of central and upwind numerical fluxes and for the two local polynomial degrees given by setting $N_{D G}=1$ and 2 in the DG approximation.

First, we verify the ability of the RK-Hermite-DG method to reproduce the whistler instability, i.e., the exponential growth of the electromagnetic whistler wave from an initial small perturbation. To this end, we monitor the time evolution of the first magnetic field Fourier mode $\hat{B}_{z}(k)$ with $k=1$ and

$$
\hat{B}_{z}(k)=\sum_{I} B_{z}\left(x_{c}^{I}\right) e^{i k x_{c}^{I}}
$$

where $x_{c}^{I}$ is the center of the $I$-th DG cell. The evolution of $\hat{B}_{z}(1)$ is shown in the left panel of Figure 5 . The whistler wave grows exponentially with the theoretically predicted growth rate $\gamma=0.035$, and later saturates due to nonlinear effects. In the right panel of Figure 5, the evolution of different energy contributions

$$
\frac{\Delta \mathcal{E}_{E B}}{\mathcal{E}(0)}=\frac{\mathcal{E}_{E B}(t)-\mathcal{E}_{E B}(0)}{\mathcal{E}(0)}, \quad \frac{\Delta \mathcal{E}_{\text {kin }}^{s}}{\mathcal{E}(0)}=\frac{\mathcal{E}_{\text {kin }}^{s}(t)-\mathcal{E}_{\text {kin }}^{s}(0)}{\mathcal{E}(0)}, \quad \frac{\Delta \mathcal{E}}{\mathcal{E}(0)}=\frac{\mathcal{E}(t)-\mathcal{E}(0)}{\mathcal{E}(0)},
$$

is plotted, where

$$
\mathcal{E}_{E B}(t)=\frac{1}{2}\left(\frac{\omega_{c e}}{\omega_{p e}}\right)^{2} \int_{\Omega_{x}}\left(\mathbf{E}^{2}+\mathbf{B}^{2}\right) d \boldsymbol{x}, \quad \mathcal{E}_{\text {kin }}^{s}(t)=\frac{m^{s}}{2} \int_{\Omega_{x}} \int_{\Omega_{v}} v^{2} f^{s} d \boldsymbol{x} d \boldsymbol{v}, \quad \mathcal{E}(t)=\mathcal{E}_{E B}(t)+\sum_{s} \mathcal{E}_{k i n}^{s}(t),
$$

are the electromagnetic energy, the kinetic energy of species $s$, and the total energy, respectively.

The energy of the electromagnetic wave grows at the expense of the kinetic energy of the electrons, while the ion kinetic energy stays almost unchanged. This is expected since the whistler instability is controlled by the electron dynamics, and ions are effectively motionless on the short electron time scales. Our simulations produce almost indistinguishable results for two $N_{D G}=1$ and 2, central and upwind numerical fluxes in the discretization of the Maxwell equations, and time steps $\Delta t=0.02,0.01,0.005$. For this reason, in Figure 5 , we report only the numerical results when using the central numerical flux, $\Delta t=0.02$, and $N_{D G}=1,2$ (left panel) and $N_{D G}=2$ (right panel). 

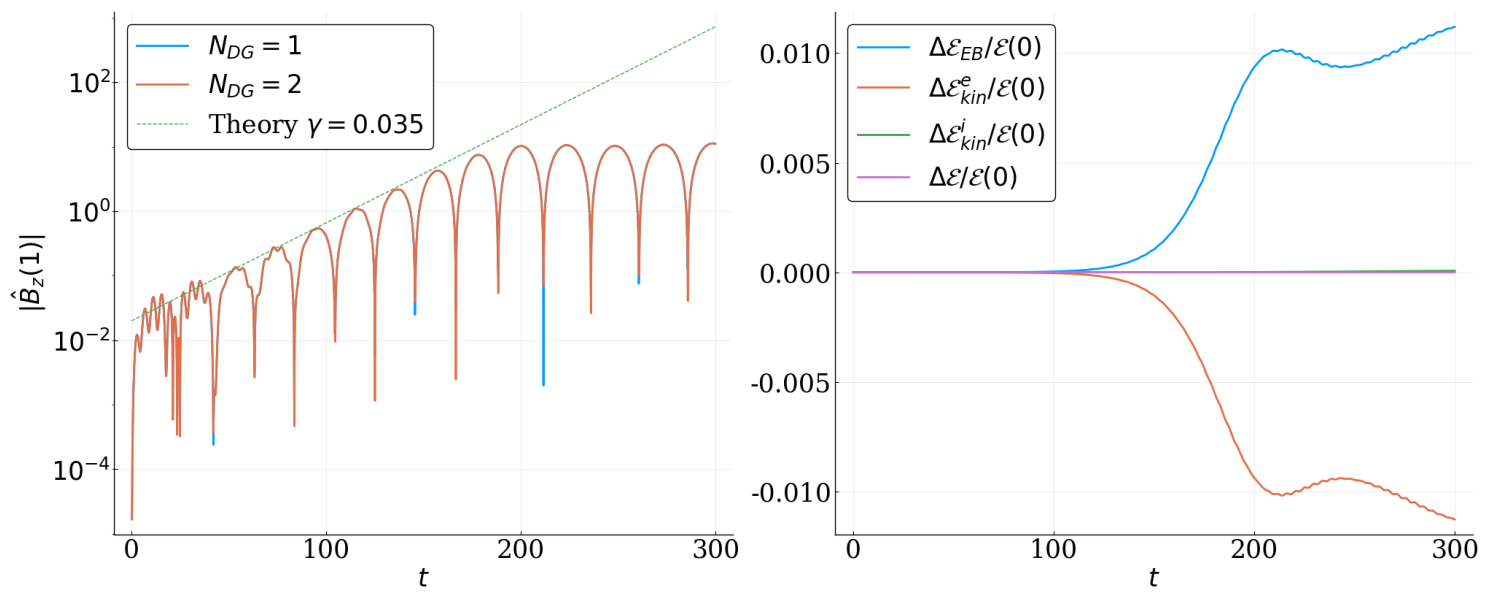

Fig. 5. Whistler instability benchmark: time evolution of $\left|\hat{B}_{z}(1)\right|$ (left panel); time evolution of electromagnetic, kinetic, and total energy (right panel). Numerical results are shown when using the central numerical flux, $\Delta t=0.02$, and $N_{D G}=1,2$ (left panel) and $N_{D G}=2$ (right panel).

\subsection{Tearing instability}

The next benchmark problem concerns the evolution of the so-called tearing instability, which is a linear instability that produces magnetic reconnection in sheared magnetic field configurations, e.g. [11]. The tearing instability (and magnetic reconnection in general) exists only in the presence of finite dissipation, which in collisionless plasmas is produced by kinetic effects associated with wave-particle interactions. As such, it represents a challenging and practically important illustration of the method's ability to correctly capture kinetic effects. Furthermore, relative to the whistler instability, which in practice involves only electron motion, this test is truly multi-scale, since both electrons and ions concur to the development of the tearing instability.

We employ a Harris sheet equilibrium [50], adapted to satisfy periodic boundary conditions by initializing two separate reconnection regions. For this purpose, we initialize four different plasma species (electrons and ions for each reconnection region) with distribution functions

$$
f^{s}(\boldsymbol{x}, \boldsymbol{v}, 0)=\frac{n^{s}(\boldsymbol{x})}{(2 \pi)^{3 / 2} v_{T_{s}}^{3}} \exp \left[-\frac{v_{x}^{2}+v_{y}^{2}+\left(v_{z}-V_{z}^{s}\right)^{2}}{2 v_{T_{s}}^{2}}\right],
$$

where $s \in\{$ electrons $\# 1$, electrons \#2, ions \#1, ions \#2 $\}$ denoted by $e 1, e 2, i 1, i 2$, respectively. In this case, the initial plasma density is

$$
\begin{aligned}
& n^{e 1}(\boldsymbol{x})=n^{i 1}(\boldsymbol{x})=\operatorname{sech}^{2}\left(\frac{x-0.25 L_{x}}{\lambda}\right), \\
& n^{e 2}(\boldsymbol{x})=n^{i 2}(\boldsymbol{x})=\operatorname{sech}^{2}\left(\frac{x-0.75 L_{x}}{\lambda}\right),
\end{aligned}
$$

and current sheets of width $\lambda$ are created by counterstreaming electrons and ions. Moreover, current sheets at $x=0.25 L_{x}$ and $x=0.75 L_{x}$ are initialized in opposite directions, i.e., $V_{z}^{e 1}=-V_{z}^{e 2}=-1 /(32 \sqrt{3})$ and $V_{z}^{i 1}=-V_{z}^{i 2}=1 /(16 \sqrt{3})$. Additionally, the equilibrium magnetic field is initialized to satisfy the stationary Ampère law

$$
B_{x}=0, \quad B_{y}=\tanh \left(\frac{x-0.25 L_{x}}{\lambda}\right)-\tanh \left(\frac{x-0.75 L_{x}}{\lambda}\right)-1, \quad B_{z}=1 .
$$

The equilibrium configuration has the following dimensionless parameters

$$
\frac{T_{i}}{T_{e}}=2, \quad \frac{\omega_{p e}}{\omega_{c e}}=2, \quad \frac{m_{i}}{m_{e}}=256, \quad \frac{v_{T_{i}}}{\omega_{c i} \lambda}=1,
$$


which are sufficient to reconstruct the other equilibrium parameters.

In order to initiate reconnection, we seed an unstable perturbation in the magnetic field as

$$
\delta B_{x}=-\delta B k_{y} \sin \left(k_{x} x\right) \sin \left(k_{y} y\right), \quad \delta B_{y}=-\delta B k_{x} \cos \left(k_{x} x\right) \cos \left(k_{y} y\right), \quad \delta B_{z}=0,
$$

with $\delta B=10^{-3}, k_{x}=2 \pi / L_{x}$, and $k_{y}=2 \pi / L_{y}$.

We discretize the velocity space by setting $N_{v_{x}}=N_{v_{y}}=N_{v_{z}}=9$, so to have 10 polynomials in each velocity direction. The physical space domain $\Omega_{x}=\left[0, L_{x}\right] \times\left[0, L_{y}\right] \times\left[0, L_{z}\right]$ is chosen with $L_{x}=200$, $L_{y}=4 \pi \lambda=64 \pi / \sqrt{3}, L_{z}=1$ and discretized with $N_{x}=128, N_{y}=36, N_{z}=1, N_{D G}=2$ with uniform cell size in every direction. Lastly, we use $\nu=1$ to avoid filamentation of the particle distribution function and the third order adaptive RK scheme of Bogacki-Shampine [13] with average $\Delta t \approx 0.14$ and final simulation time $T=10^{5}$.

First, we verify the ability of SPS-DG to reproduce the tearing instability, i.e., the exponential growth of the excited perturbation (97) relative to the growth rate $\gamma=1.13 \cdot 10^{-4}$ computed by a linear Vlasov solver [46]. (This growth rate was also verified with a different linear solver based on an Hermite expansion of the linearized Vlasov-Maxwell equations [21].) To this end, we monitor the time evolution of the magnetic field Fourier mode $\hat{B}\left(k_{x}, k_{y}\right)$ with $k_{x}=0, k_{y}=1$ and

$$
\begin{gathered}
\hat{B}\left(k_{x}, k_{y}\right)=\frac{1}{N_{x} N_{y}} \sqrt{\sum_{\beta \in\{x, y, z\}} \hat{B}_{\beta}^{2}\left(k_{x}, k_{y}\right)}, \\
\hat{B}_{\beta}\left(k_{x}, k_{y}\right)=\sum_{I / 2} B_{\beta}\left(x_{c}^{I}, y_{c}^{I}\right) e^{i k_{x} x_{c}^{I}} e^{i k_{y} y_{c}^{I}},
\end{gathered}
$$

where summation $\sum_{I / 2}$ is performed over half of the domain, i.e., $\left[0, L_{x} / 2\right] \times\left[0, L_{y}\right]$, so that only one current sheet is considered (otherwise the $k_{x}=0$ mode cancels out due to symmetry). The evolution of $\hat{B}(0,1)$ is shown in Figure 6 where one can see that the tearing instability grows exponentially with a growth rate that is in good agreement with the theoretically predicted value, and later saturates due to nonlinear effects. The growth rate from the SPS-DG simulation is $\gamma=1.09 \times 10^{-4}$, with a $4 \%$ relative error with respect to the value obtained by numerically solving the linearized Vlasov-Maxwell equations, $\gamma=1.13 \times 10^{-4}$. We note that the latter computation is performed for a single current sheet configuration and some (minor) difference with the double-sheet configuration is expected given that the eigenfunction of the tearing instability decays weakly with distance from the center of the current sheet and in the considered configuration the current sheets are separated only by the distance of approximately $10.8 \lambda$. 


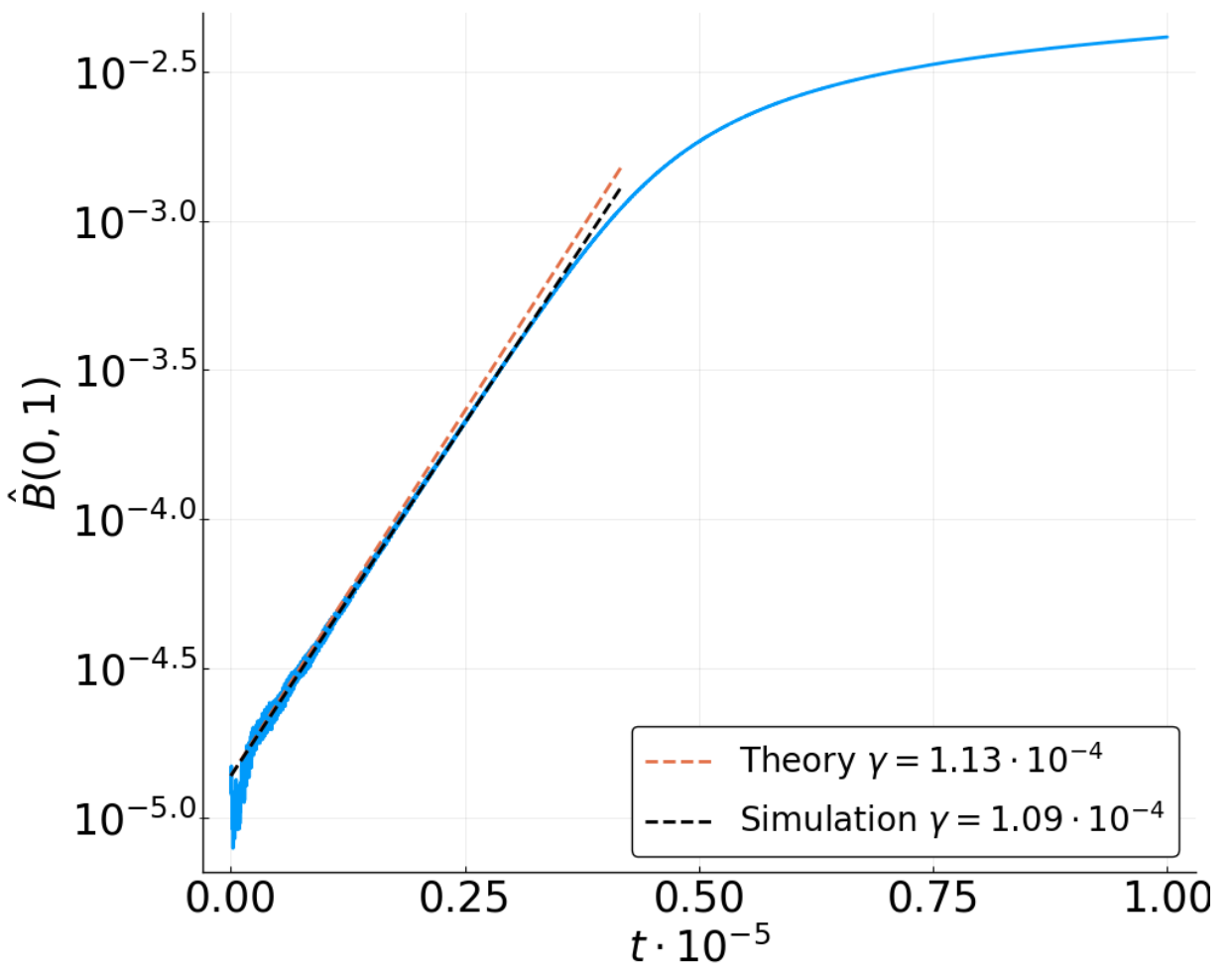

Fig. 6. Tearing instability benchmark: time evolution of unstable mode $\hat{B}(0,1)$.

The current densities for the initial time $t=0$ and the final time $t=10^{5}$ are shown in Fig. 7 . The figure shows only half of the simulation domain, i.e., $\left[0, L_{x} / 2\right] \times\left[0, L_{y}\right]$, since the other half is symmetric. One can see that the initial uniform current sheet has evolved forming the so-called X-point, which is the characteristic signature of magnetic reconnection.
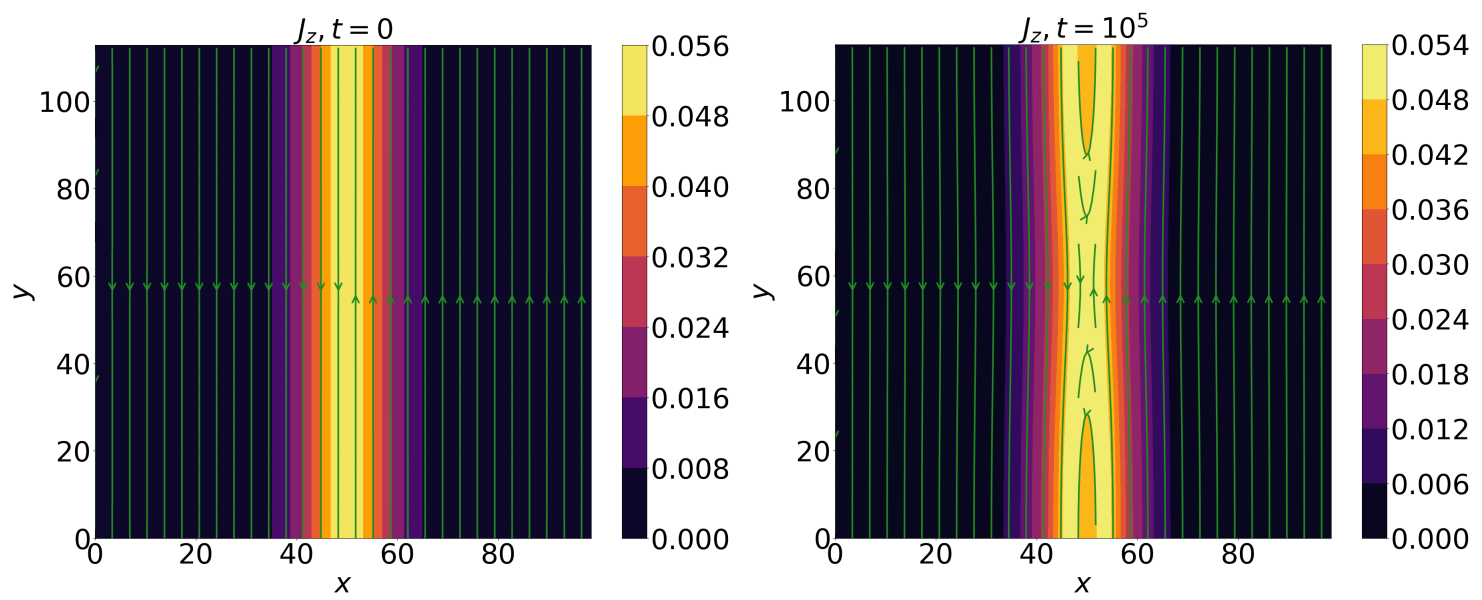

Fig. 7. Tearing instability benchmark: current density $j_{z}$ along $\hat{\mathbf{z}}$ axis in the simulations at initial $t=0$ (left panel) and final time $t=10^{5}$ (right panel). Green contour lines show the direction of magnetic field lines. 


\subsection{Orszag-Tang vortex}

The last benchmark is the Orszag-Tang vortex problem [70]. Here the initial condition corresponds to two large-scale vortices, which subsequently evolve to form small-scale structures, such as current sheets. If the system size is sufficiently large, a transition to fully developed turbulence occurs via breaking of thin current sheets by magnetic reconnection. In fact, the Orszag-Tang initial conditions are often used in studies of two-dimensional plasma turbulence, see e.g. [12, 72, 85] and others. The problem is of particular interest for the present work, since it is an example of the interaction between large-scale, fluid-like behavior and small-scale, dissipative processes involving kinetic physics. We compare solutions obtained using the SPSDG method against a reference solution obtained using a conventional PIC algorithm implemented in the VPIC code $[14,15,16]$. In principle, the model equations considered in the SPS-DG and VPIC codes are different, because the VPIC code solves the relativistic version of the Vlasov-Maxwell system, while SPS-DG does not account for relativistic effects. However, we will consider parameters where the relativistic effects are not important, so that a comparison is meaningful. Since the early evolution of the system is dominated by large-scale structures, the dynamics of the SPS-DG and VPIC solutions should be the same. Later in time, when small-scale structures form and kinetic physics becomes important, we may expect the behavior of the SPS-DG and VPIC code to differ due to the limited resolution in velocity space of SPS-DG. Further, late-time evolution may become turbulent (stochastic), so that comparisons between the solutions are only meaningful in a statistical sense.

In this test, we discretize the velocity space by setting $N_{v_{x}}=N_{v_{y}}=N_{v_{z}}=9$, and the physical space domain $\Omega_{x}=\left[0, L_{x}\right] \times\left[0, L_{y}\right] \times\left[0, L_{z}\right]$ by setting $L_{x}=L_{y}=50$ and $L_{z}=1$ and $N_{x}=N_{y}=120, N_{z}=1$, with uniform cell size in every direction. We impose spatially periodic boundary conditions. The components of the initial magnetic field $\boldsymbol{B}(\boldsymbol{x}, 0)$ are set to

$$
\begin{aligned}
& B_{x}(\boldsymbol{x}, 0)=-\delta B \sin \left(k_{y} y+4.1\right), \\
& B_{y}(\boldsymbol{x}, 0)=\delta B \sin \left(2 k_{x} x+2.3\right), \\
& B_{z}(\boldsymbol{x}, 0)=1,
\end{aligned}
$$

with $\delta B=0.2, k_{x}=2 \pi / L_{x}, k_{y}=2 \pi / L_{y}$. The (randomly chosen) phases 4.1 and 2.3 are needed to remove any artificial symmetry in the initial setup. The distribution functions for electrons and ions are initialized to shifted Maxwellian distributions with spatially uniform density (we omit species superscripts for clarity):

$$
f(\boldsymbol{x}, \boldsymbol{v}, 0)=\prod_{\beta \in\{x, y, z\}} \frac{1}{v_{T \beta} \sqrt{2 \pi}} \exp \left[-\frac{\left(v_{\beta}-V_{\beta}(\boldsymbol{x})\right)^{2}}{2 v_{T_{\beta}}^{2}}\right],
$$

with electron and ion velocities

$$
\begin{aligned}
V_{x}^{e}(\boldsymbol{x}) & =-\delta B v_{a} \sin \left(k_{y} y+0.5\right), \\
V_{y}^{e}(\boldsymbol{x}) & =\delta B v_{a} \sin \left(k_{x} x+1.4\right), \\
V_{z}^{e}(\boldsymbol{x}) & =-\frac{\delta B \omega_{c e}}{\omega_{p e}}\left(2 k_{x} \cos \left(2 k_{x} x+2.3\right)+k_{y} \cos \left(k_{y} y+4.1\right)\right), \\
V_{x}^{i}(\boldsymbol{x}) & =U_{x}^{e}(\boldsymbol{x}), \\
V_{y}^{i}(\boldsymbol{x}) & =U_{y}^{e}(\boldsymbol{x}), \\
V_{z}^{i}(\boldsymbol{x}) & =0
\end{aligned}
$$

where $v_{a}=0.1$ and $\omega_{p e} / \omega_{c e}=2$. The values 0.5 and 1.4 above are randomly chosen phases and $V_{z}^{e}$ is set to satisfy Ampère's law at time $t=0$. Other parameters include $m_{i} / m_{e}=25$ and $\nu=1$. The shifted Maxwellian distribution is initialized according to the formula

$$
C_{n, m, p}=\frac{1}{\alpha} \sqrt{\frac{2^{n} 2^{m} 2^{p}}{n ! m ! p !}}\left(\frac{V_{x}}{\alpha_{x}}\right)^{n}\left(\frac{V_{y}}{\alpha_{y}}\right)^{m}\left(\frac{V_{z}}{\alpha_{z}}\right)^{p}
$$

where $\alpha_{\beta}^{e}=\sqrt{2} v_{T_{\beta}}^{e}=0.25, u_{\beta}^{e}=0$ and $\alpha_{\beta}^{i}=\sqrt{2} v_{T_{\beta}}^{e} / \sqrt{m_{i} / m_{e}}=0.05, u_{\beta}^{i}=0$ with $\beta \in\{x, y, z\}$ for electrons and ions, respectively. 
For comparison with VPIC, we consider three different local discontinuous polynomial degrees, $N_{D G}=$ $1,2,3$, and both central and upwind flux formulas in the DG approximation of the Maxwell equations. We advance the numerical solution in time by using the adaptive third-order accurate RK scheme of BogackiShampine with second order embedded method [13], where the mean time steps are $\Delta t=0.13, \Delta t=0.079$, $\Delta t=0.053$, for $N_{D G}=1, N_{D G}=2, N_{D G}=3$, respectively. The reference VPIC solution is computed on a grid with $N_{x}=N_{y}=880, N_{z}=1$, equally spaced partitions of the physical space domain $\Omega_{x}$, time step $\Delta t=0.039373$ and average number of particles per cell $N_{p}=4000$. The simulations run for two Alfvén times, i.e., up to $T=2 L_{x} / v_{a}=1000$, where the Alfvén time, $L_{x} / v_{a}$, is a characteristic dynamic time scale of the system.

The currents along $\hat{\mathbf{z}}$ formed after one Alfvén time (at $t=500$ ) are shown in Figure 8 for SPS-DG with $N_{D G}=1,2,3$ and for PIC. The figure shows a good agreement between all SPS-DG runs and the fully kinetic PIC algorithm, indicating that even a spatial discretization with $N_{D G}=1$ is sufficient in the early times of the simulation.
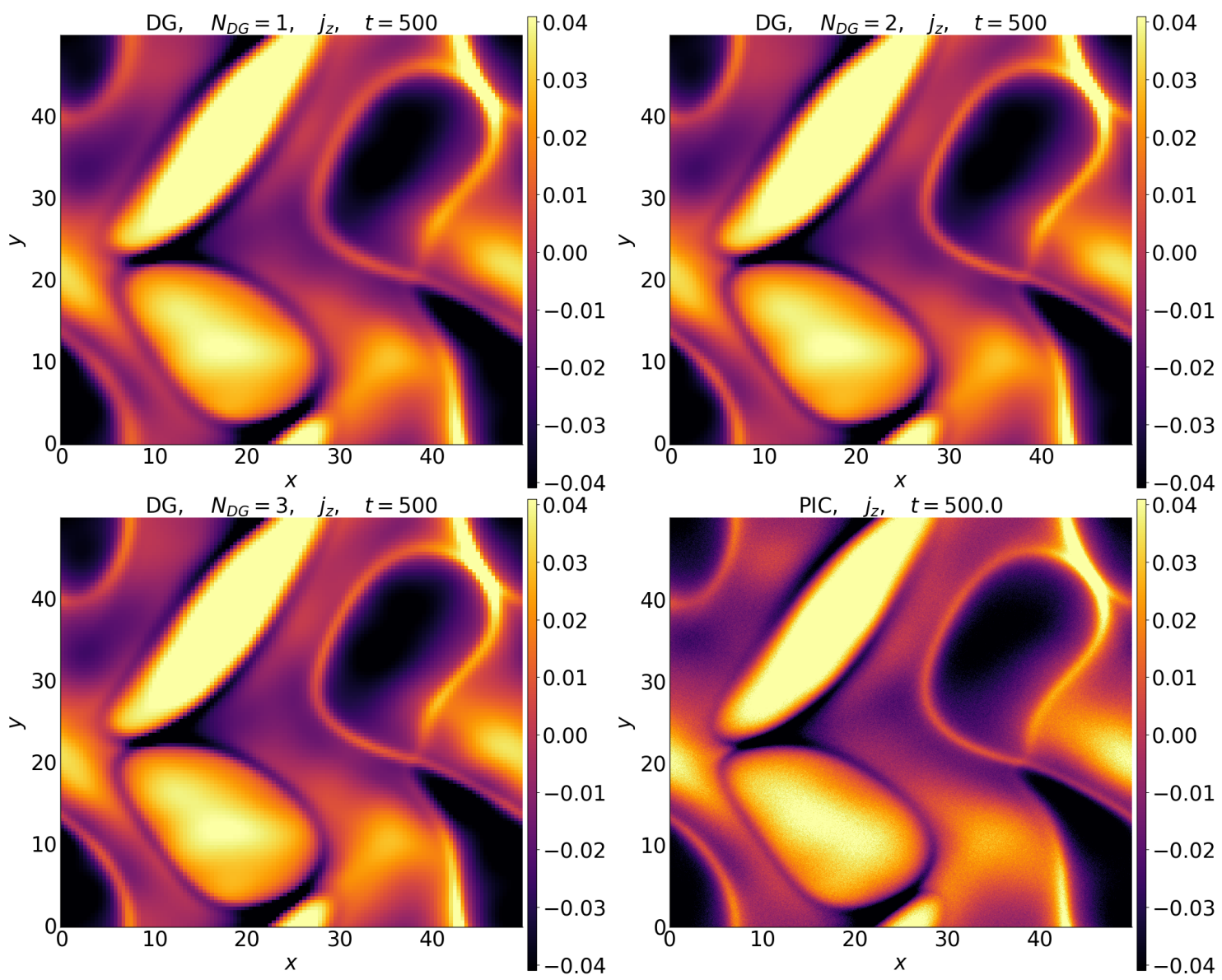

Fig. 8. Orszag-Tang vortex benchmark: current density $j_{z}$ along $\hat{\mathbf{z}}$ axis in the simulations at one Alfvén time $t=L_{x} / v_{a}=500$.

Further evolution of the current density along $\hat{\mathbf{z}}$ is shown in Figure 9 where time snapshot $t=1000$ is shown for SPS-DG with $N_{D G}=3$ and for PIC. We can see that the agreement between the two numerical solutions decreases with time, with SPS-DG becoming slightly more diffusive. We attribute this effect to the lack of velocity space resolution, because strong flows form at later times making the plasma distribution function strongly non-Maxwellian and requiring a larger number of Hermite moments to capture the smaller structures. This interpretation is also supported by the fact that the SPS-DG solutions for $N_{D G}=2$ and $N_{D G}=3$ are essentially identical (not shown), indicating that the spatial and temporal resolution is sufficient in SPS-DG (the higher order DG with $N_{D G}=3$ has smaller time step than that for $N_{D G}=2$ ). 

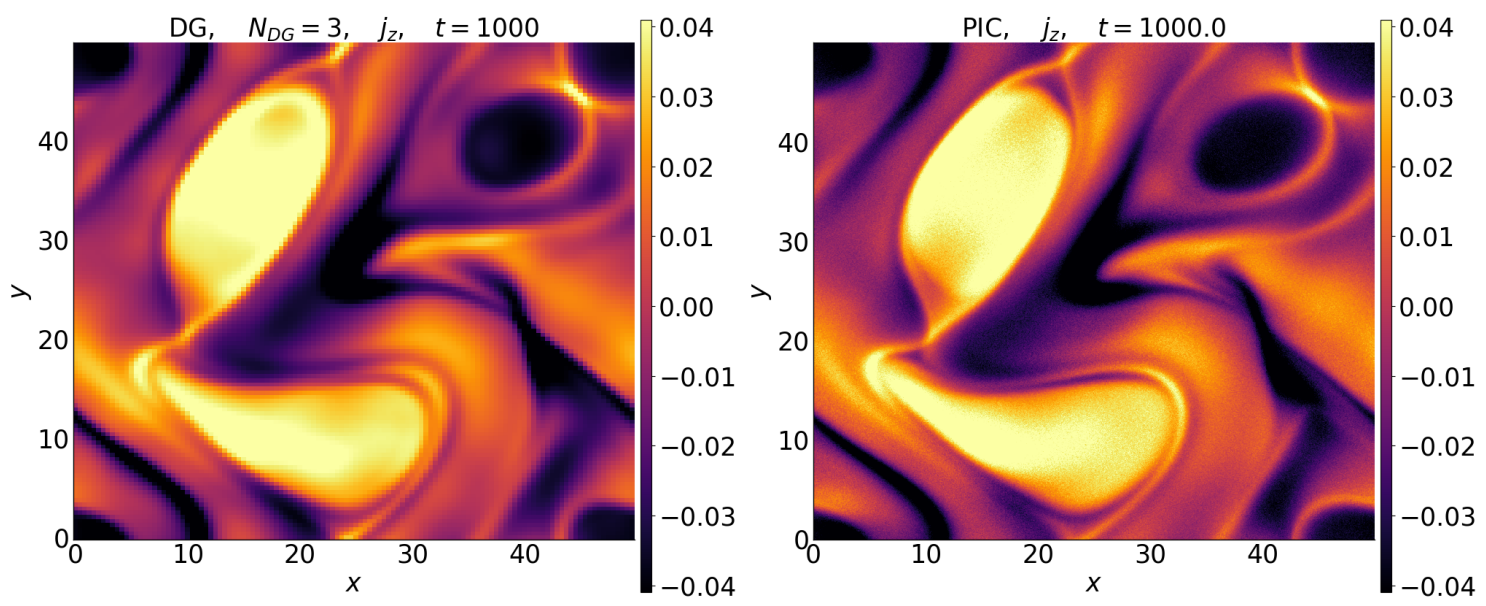

Fig. 9. Orszag-Tang vortex benchmark: current density $j_{z}$ along $\hat{\mathbf{z}}$ axis in the simulations at time $t=1000$.

Next, we compare the omnidirectional spectrum of the magnetic field in Figure 10. The spectra agree very well (up $k \sim 10$, for higher $k$ the PIC spectrum is dominated by particle noise) at early times and start to diverge slightly at later times $\left(t \sim 1500\right.$, not shown) for $N_{D G} \geq 2$. The case $N_{D G}=1$ is too diffusive and the spectra start to diverge at $k \sim 1$. The results for SPS-DG with $N_{D G}=2$ and $N_{D G}=3$ practically coincide, confirming that the chosen spatial and temporal resolution in SPS-DG is sufficient in these cases.
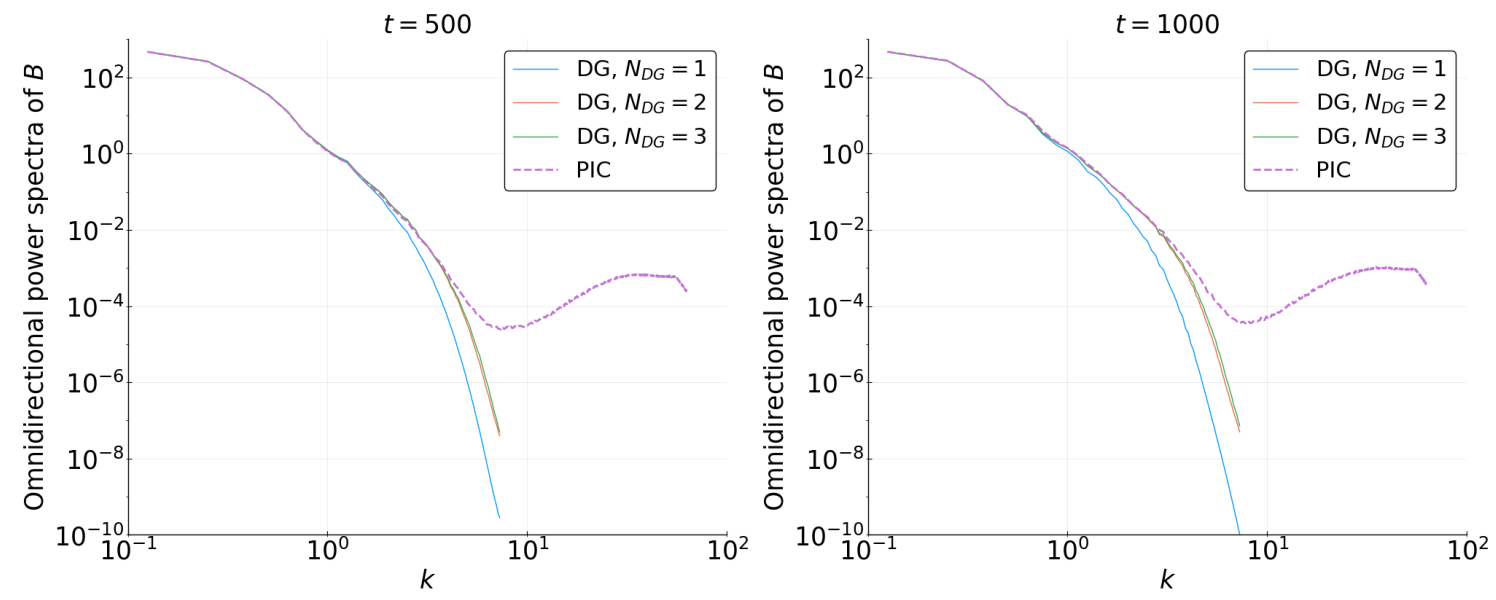

Fig. 10. Orszag-Tang vortex benchmark: Omnidirectional power spectrum of magnetic fluctuations in the simulations at times $t=500,1000$.

An important part of the comparison between SPS-DG and PIC is the evolution of the energies defined in Eqs. (90) and (91). This comparison is shown in Figure 11, which shows the various parts of the total energy normalized to the energy of the initial perturbation $\left(\mathcal{E}_{\text {pert }}\right)$, which includes the contribution of $B_{x}, B_{y}, U_{\beta}^{e}, U_{\beta}^{i}$ for $\beta \in\{x, y, z\}$. The evolution of the electromagnetic energy in SPS-DG closely follows PIC with slight differences at later times. The comparison of the kinetic energy reveals a good agreement as well, with slight differences for electrons at later times. Part of this disagreement can be attributed to relativistic effects in the PIC simulations. Indeed, particles kinetic energies in the relativistic case are bigger than in the nonrelativistic case by the Lorentz factor. To estimate relativistic heating, we compare the measured thermal relativistic energy in the PIC simulations with the classical non-relativistic estimate (i.e., $3 T / 2$ with $T$ the temperature). The difference for electrons is $10 \%$ and for ions $0.4 \%$, which is consistent with the slight differences between electron kinetic and electromagnetic energies between SPS-DG and PIC. 

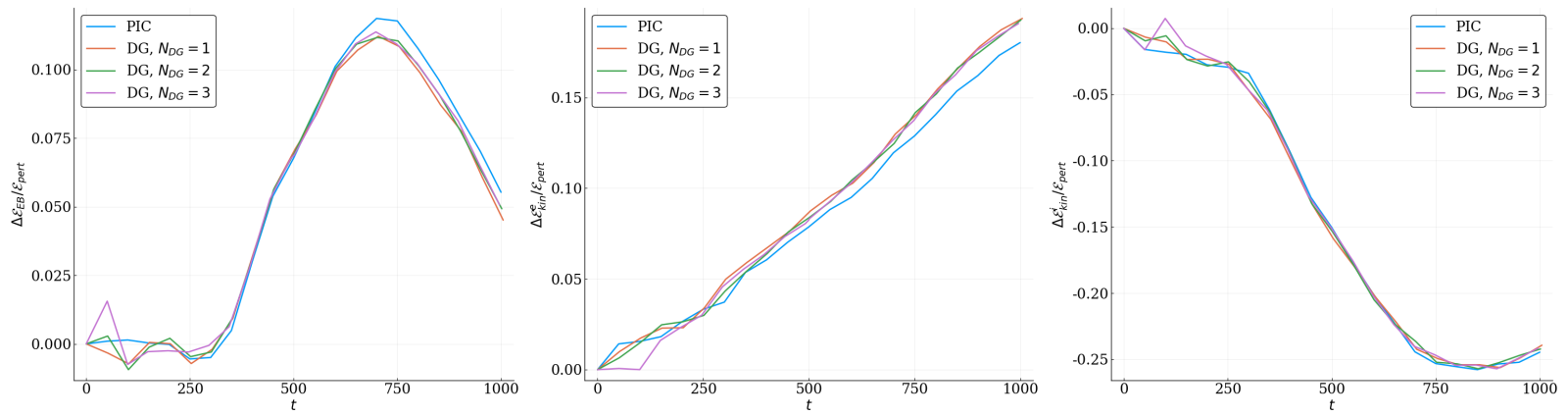

Fig. 11. Energy evolution in Orszag-Tang vortex test.

In summary, the comparison between SPS-DG and PIC on the OT test problem reveals that, while some small-scale local differences exist between the two at later times, 10 Hermite moments in each direction are sufficient to capture accurately the early-time dynamics of the system as well as the behavior of important quantities such as magnetic-field spectra and energy partition.

\section{Conclusions}

We have presented a new spectral method for the solution of the multi-dimensional Vlasov-Maxwell equations. The method combines an Hermite expansion in velocity space with a discontinuous Galerkin discretization for the spatial coordinates. In terms of DG, we analyze two schemes differing by the treatment of the flux at the interface between cells. Specifically, the Vlasov equation is discretized with upwind fluxes while Maxwell's equations can be discretized either with upwind or central fluxes. While upwind fluxes tend to improve the overall stability of the scheme, using central fluxes in Maxwell's equations can lead to the conservation of total energy in the system (under appropriate boundary conditions). We have further adopted an explicit time discretization based on various Runge-Kutta methods of different orders.

The algorithms described in this paper have been implemented in the SPS-DG code. SPS-DG takes advantage of the PETSc data structure and solvers. Several numerical tests have been presented to show (a) the nearly optimal scalability of the approach up to $\sim 40,000$ cores, and (b) the accuracy of the spatial discretization, where we have recovered the appropriate order of convergence of the different DG schemes with a method of manufactured solutions. Additional tests like the whistler and tearing instabilities and the Orszag-Tang turbulence cascade demonstrate the successful application of the method to standard plasma physics problems and suggest that the method can capture kinetic behavior even with a relatively low number of modes per direction.

Spectral methods with a suitable spectral basis, like the one considered in this paper, feature built-in fluidkinetic coupling, i.e. they can capture the macroscopic dynamics of magnetized plasmas with the low-order moments of the expansion while the kinetic physics can be retained by adding higher-order moments only where necessary (i.e. locally in space and time). The DG discretization adds the ability to handle sharp, shock-like structures and extreme data locality to enable scalable implementations on high-performancecomputing architectures. This removes the performance limitations of some of the earlier implementations of the Hermite spectral method, which was coupled with a Fourier spatial discretization and was hence limited in parallel scalability by the FFTs global communications. In the future, these new algorithms might therefore enable simulations of the large-scale plasma dynamics with accurate feedback from the microscopic physics.

\section{Acknowledgments}

The work of GLD, OK, GM was supported by the Laboratory Directed Research and Development Exploratory and Research (LDRD-ER) Program of Los Alamos National Laboratory under project number 
20170207ER. Los Alamos National Laboratory is operated by Triad National Security, LLC, for the National Nuclear Security Administration of U.S. Department of Energy (Contract No. 89233218CNA000001). Computational resources for the SPS-DG simulations were provided by the Los Alamos National Laboratory Institutional Computing Program. VR's contributions were supported by NASA grant NNX15AR16G. Computational resources for PIC simulations were provided by the NASA High-End Computing Program through the NASA Advanced Supercomputing Division at Ames Research Center and by the Blue Waters sustained-petascale computing project, which is supported by the National Science Foundation (awards OCI0725070 and ACI-1238993) and the state of Illinois. Blue Waters allocation was provided by the National Science Foundation through PRAC award 1614664.

\section{References}

[1] M. Abramowitz and I. A. Stegun. Vector-Addition Coefficients. In Handbook of Mathematical Functions with Formulas, Graphs, and Mathematical Tables, pages 1006-1010. Dover, New York, 1972.

[2] T. P. Armstrong, R. C. Harding, G. Knorr, and D. Montgomery. Solution of Vlasov's equation by transform methods. Methods in Computational Physics, 9:29-86, 1970.

[3] B. Ayuso, J. A. Carrillo, and C.-W. Shu. Discontinuous Galerkin methods for the one-dimensional Vlasov-Poisson system. Kintetic and Related Models, 4(4):955-989, 2011.

[4] B. Ayuso, J. A. Carrillo, and C.-W. Shu. Discontinuous Galerkin methods for the multi-dimensional Vlasov-Poisson problem. Mathematical Models and Methods in Applied Sciences, 22(12):1250042, 2012.

[5] B. Ayuso and S. Hajan. High-order and energy preserving discontinuous Galerkin methods for the Vlasov-Poisson system. Technical Report arXiv:1209.4025v2, arXiv, 2012.

[6] S. Balay et al. PETSc FAQ. https://www.mcs.anl.gov/petsc/documentation/faq.html\# slowerparallel. Accessed: 2019-06-24.

[7] S. Balay et al. PETSc users manual. Technical Report ANL-95/11 - Revision 3.9, Argonne National Laboratory, 2018.

[8] S. Balay et al. PETSc Web page. http://www.mcs.anl.gov/petsc, 2018.

[9] S. Balay, W. D. Gropp, L. Curfman McInnes, and B. F. Smith. Efficient management of parallelism in object oriented numerical software libraries. In Modern Software Tools in Scientific Computing, pages 163-202. Birkhäuser Press, 1997.

[10] N. Besse, F. Berthelin, Y. Brenier, and P. Bertrand. The multi-water-bag equations for collisionless kinetic modeling. Kinet. Relat. Models, 2(1):39-80, 2009.

[11] D. Biskamp. Magnetic Reconnection in Plasmas. Cambridge University Press, Cambridge, 2000.

[12] D. Biskamp and H. Welter. Dynamics of decaying two-dimensional magnetohydrodynamic turbulence. Physics of Fluids B: Plasma Physics, 1(10):1964-1979, 1989.

[13] P. Bogacki and L. F. Shampine. A 3(2) pair of Runge-Kutta formulas. Applied Mathematics Letters, 2(4):321-325, 1989.

[14] K. J. Bowers, B. J. Albright, B. Bergen, L. Yin, K. J. Barker, and D. J. Kerbyson. 0.374 Pflop/s trillion-particle kinetic modeling of laser plasma interaction on Roadrunner. In Proceedings of the 2008 ACM/IEEE conference on Supercomputing, page 63. IEEE Press, 2008.

[15] K. J. Bowers, B. J. Albright, L. Yin, B. Bergen, and T.J.T. Kwan. Ultrahigh performance threedimensional electromagnetic relativistic kinetic plasma simulation. Physics of Plasmas, 15(5):055703, 2008.

[16] K. J. Bowers, B. J. Albright, L. Yin, W. Daughton, V. Roytershteyn, B. Bergen, and T.J.T. Kwan. Advances in petascale kinetic plasma simulation with VPIC and Roadrunner. Journal of Physics: Conference Series, 180(1):012055, 2009.

[17] A. J. Brizard and T. S. Hahm. Foundations of nonlinear gyrokinetic theory. Rev. Mod. Phys., 79:421468, Apr 2007.

[18] Zhenning Cai, Ruo Li, and Yanli Wang. Solving vlasov equations using nr\$xx\$ method. SIAM Journal on Scientific Computing, 35(6):A2807-A2831, 2013.

[19] Zhenning Cai and Yanli Wang. Suppression of recurrence in the hermite-spectral method for transport 
equations. SIAM Journal on Numerical Analysis, 56(5):3144-3168, 2018.

[20] E. Camporeale, G. L. Delzanno, B. K. Bergen, and J. D. Moulton. On the velocity space discretization for the Vlasov-Poisson system: comparison between implicit Hermite spectral and Particle-in-Cell methods. Computer Physics Communications, 198:47-58, 2015.

[21] E. Camporeale, G. L. Delzanno, G. Lapenta, and W. Daughton. New approach for the study of linear Vlasov stability of inhomogeneous systems. Physics of Plasmas, 13(9):092110, 2006.

[22] A. Cangiani, Z. Dong, E. H. Georgoulis, and P. Houston. hp-version viscontinuous Galerkin methods on polygonal and polyhedral meshes. Springer International Publishing, first edition, 2017.

[23] J. Canosa, J. Gazdag, and J. E. Fromm. The recurrence of the initial state in the numerical solution of the vlasov equation. Journal of Computational Physics, 15(1):34 - 45, 1974.

[24] Y. Chen, G. Tóth, P. Cassak, X. Jia, T. I. Gombosi, J. A. Slavin, S. Markidis, I. B. Peng, V. K. Jordanova, and M. G. Henderson. Global three-dimensional simulation of Earth's dayside reconnection using a two-way coupled magnetohydrodynamics with embedded particle-in-cell model: Initial results. Journal of Geophysical Research: Space Physics, 122(10):10,318-10,335, 2017.

[25] C. Z. Cheng and G. Knorr. The integration of the Vlasov equation in configuration space. Journal of Computational Physics, 22(3):330 - 351, 1976.

[26] Y. Cheng, A. J. Christlieb, and X. Zhong. Energy-conserving discontinuous Galerkin methods for the Vlasov-Maxwell system. Journal of Computational Physics, 279:145-173, 2014.

[27] Y. Cheng, I. Gamba, F. Li, and P. Morrison. Discontinuous Galerkin methods for the Vlasov-Maxwell equations. SIAM Journal on Numerical Analysis, 52(2):1017-1049, 2014.

[28] Y. Cheng, I. Gamba, A. Majorana, and C.-W. Shu. A discontinuous Galerkin solver for BoltzmannPoisson systems in nano devices. Computer Methods in Applied Mechanics and Engineering, 198(3440):31303150, 2009.

[29] Y. Cheng, I. M. Gamba, and A. Majorana. Discontinuous Galerkin solver for Boltzmann-Poisson transients. Journal of Computational Electronics, 7(3):119, 2008.

[30] Y. Cheng, I. M. Gamba, A. Majorana, and C.-W. Shu. A brief survey of the discontinuous Galerkin method for the Boltzmann-Poisson equations. SeMA Journal, 54(1):47, 2011.

[31] Y. Cheng, M. I. Gamba, and J. Proft. Positivity-preserving discontinuous Galerkin schemes for linear Vlasov-Boltzmann transport equations. Mathematics of Computation, 81(277):153-190, 2012.

[32] B. Cockburn, G. E. Karniadakis, and C.-W. Shu. The development of discontinuous Galerkin methods. volume 11 of Lect. Notes Comput. Sci. Eng., pages 3-50. Springer, Berlin, 2000.

[33] B. Cockburn, F. Li, and C.-W. Shu. Locally divergence-free discontinuous Galerkin methods for the Maxwell equations. Journal of Computational Physics, 194(2):588-610, 2004.

[34] B. Cockburn, S. Y. Lin, and C.-W. Shu. TVB Runge-Kutta local projection discontinuous Galerkin finite element method for conservation laws. III. One-dimensional systems. Journal of Computational Physics, 84(1):90-113, 1989.

[35] B. Cockburn and C.-W. Shu. TVB Runge-Kutta local projection discontinuous Galerkin finite element method for conservation laws. II. General framework. Mathematics of Computation, 52(186):411-435, 1989.

[36] B. Cockburn and C.-W. Shu. The Runge-Kutta local projection $P^{1}$-discontinuous-Galerkin finite element method for scalar conservation laws. RAIRO Modélisation Mathématique et Analyse Numérique, 25(3):337-361, 1991.

[37] L. K. S. Daldorff, G. Tóth, T. I. Gombosi, G. Lapenta, J. Amaya, S. Markidis, and J. U. Brackbill. Two-way coupling of a global Hall magnetohydrodynamics model with a local implicit particle-in-cell model. Journal of Computational Physics, 268:236-254, 2014.

[38] G. L. Delzanno. Multi-dimensional, fully-implicit, spectral method for the Vlasov-Maxwell equations with exact conservation laws in discrete form. Journal of Computational Physics, 301:338-356, 2015.

[39] G. L. Delzanno and V. Roytershteyn. High-frequency plasma waves and pitch angle scattering induced by pulsed electron beams. Journal of Geophysical Research: Space Physics, 124(9):7543-7552, 2019.

[40] Yana Di, Yuwei Fan, Zhenzhong Kou, Ruo Li, and Yanli Wang. Filtered hyperbolic moment method for the vlasov equation. Journal of Scientific Computing, 79(2):969-991, 2019.

[41] D. Fatone, L. Funaro and G. Manzini. Arbitrary-order time-accurate semi-Lagrangian spectral approx- 
imations of the Vlasov-Poisson system. J. Comput. Phys., 384:349-375, 2019.

[42] D. Fatone, L. Funaro and G. Manzini. A semi-Lagrangian spectral method for the Vlasov-Poisson system based on Fourier, Legendre and Hermite polynomials. Comm. Appl. Math. Comput., 1, 2019.

[43] E. Fehlberg. Klassische Runge-Kutta-Formeln vierter und niedrigerer Ordnung mit SchrittweitenKontrolle und ihre Anwendung auf Wärmeleitungsprobleme. Computing, 6(1):61-71, 1970.

[44] D. Funaro. Polynomial approximation of differential equations, volume 8. Springer Science \& Business Media, 2008.

[45] H. Gajewski and K. Zacharias. On the convergence of the Fourier-Hermite transformation method for the Vlasov equation with an artificial collision term. Journal of Mathematical Analysis and Applications, 61(3):752-773, 1977.

[46] S. P. Gary. Theory of space plasma microinstabilities. Cambridge University Press, 2005.

[47] R. T. Glassey. The Cauchy problem in kinetic theory, volume 52. SIAM, 1996.

[48] H. Grad. On the kinetic theory of rarefied gases. Communications on Pure and Applied Mathematics, 2(4):331-407, 1949.

[49] E. Hairer, C. Lubich, and G. Wanner. Geometric numerical integration, volume 31 of Springer Series in Computational Mathematics. Springer, Heidelberg, 2010.

[50] E. G. Harris. The equilibrium of oppositely directed magnetic fields. Nuovo Cimento, 23:115-121, 1962.

[51] J. Hesthaven and T. Warburton. Nodal Discontinuous Galerkin Methods: Algorithms, Analysis, and Applications. Springer Publishing Company, Inc., 1st edition edition, 2007.

[52] A. Ho, I. A. M. Datta, and U. Shumlak. Physics-based-adaptive plasma model for high-fidelity numerical simulations. Frontiers in Physics, 6:105, 2018.

[53] J. P. Holloway. Spectral velocity discretizations for the Vlasov-Maxwell equations. Transport Theory Stat. Phys., 25(1):1-32, 1996.

[54] G. Joyce, G. Knorr, and H. K. Meier. Numerical integration methods of the Vlasov equation. Journal of Computational Physics, 8(1):53-63, 1971.

[55] J. Juno, A. Hakim, J. TenBarge, E. Shi, and W. Dorland. Discontinuous Galerkin algorithms for fully kinetic plasmas. Journal of Computational Physics, 353:110-147, 2018.

[56] H. Karimabadi, V. Roytershteyn, H. X. Vu, Y. A. Omelchenko, J. Scudder, W. Daughton, A. Dimmock, K. Nykyri, M. Wan, D. Sibeck, M. Tatineni, A. Majumdar, B. Loring, and B. Geveci. The link between shocks, turbulence, and magnetic reconnection in collisionless plasmas. Physics of Plasmas, 21(6):062308, 2014.

[57] A. J. Klimas. A numerical method based on the Fourier-Fourier transform approach for modeling 1-D electron plasma evolution. Journal of Computational Physics, 50(2):270-306, 1983.

[58] V. I. Kolobov and R. R. Arslanbekov. Towards adaptive kinetic-fluid simulations of weakly ionized plasmas. Journal of Computational Physics, 231(3):839 - 869, 2012.

[59] O. Koshkarov, G. Manzini, G. L. Delzanno, C. Pagliantini, and V. Roytershteyn. Conservation properties of the multi-dimensional RK-Hermite-dG method for the Vlasov-Maxwell equations. Technical Report LA-UR-19-29579, Los Alamos National Laboratory, 2019.

[60] P. Lasaint and P. A. Raviart. On a finite element method for solving the neutron transport equation. In Mathematical aspects of finite elements in partial differential equations, number 33, pages 89-123, New York, 1974. Academic Press.

[61] R. J. LeVeque. Numerical Methods for Conservation Laws (2. ed.). Lectures in mathematics. Birkhuser, 1992.

[62] Y. Lin, S. Wing, J. R. Johnson, X. Y. Wang, J. D. Perez, and L. Cheng. Formation and transport of entropy structures in the magnetotail simulated with a 3-D global hybrid code. Geophysical Research Letters, 44(12):5892-5899, 2017.

[63] Alexander S. Lipatov. The Hybrid Multiscale Simulation Technology. Scientific Computation. Springer Berlin Heidelberg, Berlin, Heidelberg, 2002.

[64] N. F. Loureiro, W. Dorland, L. Fazendeiro, A. Kanekar, A. Mallet, M. S. Vilelas, and A. Zocco. Viriato: A Fourier-Hermite spectral code for strongly magnetized fluid-kinetic plasma dynamics. Computer Physics Communications, 206:45 - 63, 2016.

[65] J. Loverich, A. Hakim, and U. Shumlak. A discontinuous Galerkin method for ideal two-fluid plasma 
equations. Communications in Computational Physics, 9(2):240268, 2011.

[66] J. Loverich and U. Shumlak. A discontinuous Galerkin method for the full two-fluid plasma model. Computer Physics Communications, 169(1):251 - 255, 2005. Proceedings of the Europhysics Conference on Computational Physics 2004.

[67] G. Manzini, G. L. Delzanno, J. Vencels, and S. Markidis. A Legendre-Fourier spectral method with exact conservation laws for the Vlasov-Poisson system. Journal of Computational Physics, 317:82-107, 2016.

[68] G. Manzini, D. Funaro, and G. L. Delzanno. Convergence of spectral discretizations of the VlasovPoisson system. SIAM J. Numer. Anal., 55(5):2312-2335, 2017.

[69] J. A. Morales Escalante and I. M. Gamba. Galerkin methods for Boltzmann-Poisson transport with reflection conditions on rough boundaries. Journal of Computational Physics, 363:302, 2018.

[70] S. A. Orszag and C.-M. Tang. Small-scale structure of two-dimensional magnetohydrodynamic turbulence. Journal of Fluid Mechanics, 90(1):129-143, 1979.

[71] M. Palmroth, U. Ganse, Y. Pfau-Kempf, M. Battarbee, L. Turc, T. Brito, M. Grandin, S. Hoilijoki, A. Sandroos, and S. von Alfthan. Vlasov methods in space physics and astrophysics. Living Reviews in Computational Astrophysics, 4(1):1, 2018.

[72] T. N. Parashar, M. A. Shay, P. A. Cassak, and W. H. Matthaeus. Kinetic dissipation and anisotropic heating in a turbulent collisionless plasma. Physics of Plasmas, 16(3):32310, mar 2009.

[73] J. T. Parker and P. J. Dellar. Fourier-Hermite spectral representation for the Vlasov-Poisson system in the weakly collisional limit. Journal of Plasma Physics, 81(2):305810203, 2015.

[74] O. Pezzi, F. Valentini, S. Servidio, E. Camporeale, and P. Veltri. Fourier-Hermite decomposition of the collisional Vlasov-Maxwell system: implications for the velocity-space cascade. Plasma Physics and Controlled Fusion, 61(5):054005, 2019.

[75] W. H. Reed and T. Hill. Triangular mesh methods for the neutron transport equation. Technical Report LA-UR-73-479, Los Alamos Scientific Laboratory, 1973.

[76] V. Roytershteyn, S. Boldyrev, G. L. Delzanno, C. H. K. Chen, D. Grošelj, and N. F. Loureiro. Numerical study of inertial kinetic-Alfvén turbulence. The Astrophysical Journal, 870(2):103, 2019.

[77] V. Roytershteyn and G. L. Delzanno. Spectral approach to plasma kinetic simulations based on Hermite decomposition in the velocity space. Frontiers in Astronomy and Space Sciences, 5:27, 2018.

[78] J. W. Schumer and J. P. Holloway. Vlasov simulations using velocity-scaled Hermite representations. J. Comput. Phys., 144(2):626-661, 1998.

[79] C. W. Shu. Discontinuous Galerkin methods: General approach and stability. In Numerical Solutions of Partial Differential Equations, Adv. Courses Math. CRM Barcelona, pages 149-201. Birkhäuser, Basel, 2009.

[80] B. Srinivasan, A. Hakim, and U. Shumlak. Numerical methods for two-fluid dispersive fast mhd phenomena. Communications in Computational Physics, 10(1):183215, 2011.

[81] T. Sugiyama and K. Kusano. Multi-scale plasma simulation by the interlocking of magnetohydrodynamic model and particle-in-cell kinetic model. Journal of Computational Physics, 227(2):1340-1352, 2007.

[82] Richard Mansergh Thorne. Radiation belt dynamics: The importance of wave-particle interactions. Geophysical Research Letters, 37(22), 2010.

[83] G. Tóth, X. Jia, S. Markidis, I. B. Peng, Y. Chen, L. K. S. Daldorff, V. M. Tenishev, D. Borovikov, J. D. Haiducek, T. I. Gombosi, A. Glocer, and J. C. Dorelli. Extended magnetohydrodynamics with embedded particle-in-cell simulation of Ganymede's magnetosphere. Journal of Geophysical Research: Space Physics, 121(2):1273-1293, 2016.

[84] J. Vencels, G. L. Delzanno, A. Johnson, I. B. Peng, E. Laure, and S. Markidis. Spectral solver for multiscale plasma physics simulations with dynamically adaptive number of moments. Procedia Computer Science, 51:1148-1157, 2015.

[85] J. Vencels, G. L. Delzanno, G. Manzini, S. Markidis, I. Bo Peng, and V. Roytershteyn. SpectralPlasmaSolver: a spectral code for multiscale simulations of collisionless, magnetized plasmas. Journal of Physics: Conference Series, 719(1):012022, 2016.

[86] Juris Vencels. The Hermite-Fourier spectral method for solving the Vlasov-Maxwell system of equations. 
Master's thesis, KTH, Space and Plasma Physics, 2016.

[87] L. Wang, K. Germaschewski, A. Hakim, C. Dong, J. Raeder, and A. Bhattacharjee. Electron physics in 3-D two-fluid 10-moment modeling of Ganymede's magnetosphere. Journal of Geophysical Research: Space Physics, 123:2815-2830, 2018.

[88] L. Wang, A. Hakim, A. Bhattacharjee, and K. Germaschewski. Comparison of multi-fluid moment models with particle-in-cell simulations of collisionless magnetic reconnection. Physics of Plasmas, 22(1):012108, 2015.

[89] E. P. Wigner. On the matrices which reduce the Kronecker products of representations of SR groups. In Arthur S. Wightman, editor, The Collected Works of Eugene Paul Wigner, volume 3, pages 608-654. Springer, Berlin, 1951.

[90] D. Winske. Hybrid simulation codes with application to shocks and upstream waves. Space Science Reviews, 42(1-2):53-66, 1985.

\section{Appendix A. Derivation of (20)}

\section{A.1. Electric field terms}

We present the detailed expansion of the term containing $E_{x}$ in (20). The final formula is obtained by using the Hermite expansion of the distribution function, substituting the formulas for the derivatives of the Hermite basis functions, and exploiting the orthogonality properties. The other two terms, i.e., $E_{y}, E_{z}$, are easily obtained by a simple permutation of indices, as shown below.

$$
\int_{\Omega_{v}} E_{x}(\boldsymbol{x}, t) \frac{\partial f^{s}(\boldsymbol{x}, \boldsymbol{v}, t)}{\partial v_{x}} \psi^{n}\left(\xi_{x}^{s}\right) \psi^{m}\left(\xi_{y}^{s}\right) \psi^{p}\left(\xi_{z}^{s}\right) d \xi_{x}^{s} d \xi_{y}^{s} d \xi_{z}^{s}=-E_{x}(\boldsymbol{x}, t) C_{n-1, m, p}(\boldsymbol{x}, t) \frac{\sqrt{2 n}}{\alpha_{x}^{s}} .
$$

Term $E_{y}$ is obtained by permuting the indices $(x, y, z) \rightarrow(y, z, x)$ and $(n, m, p) \rightarrow(m, p, n)$; term $E_{z}$ is obtained by permuting the indices $(x, y, z) \rightarrow(z, x, y)$ and $(n, m, p) \rightarrow(p, n, m)$.

\section{A.2. Magnetic field terms}

The magnetic field contributes to the Lorentz force by

$$
\boldsymbol{v} \times \boldsymbol{B}=\left[v_{y} B_{z}-v_{z} B_{y},-v_{x} B_{z}+v_{z} B_{x}, v_{x} B_{y}-v_{y} B_{x}\right]^{T} .
$$

We consider the terms associated with $B_{x}$, that involve the following derivatives of the distribution function

$$
B_{x} v_{z} \partial f^{s} / \partial v_{y}, \quad-B_{x} v_{y} \partial f^{s} / \partial v_{z}
$$

The similar terms associated with $B_{y}$ and $B_{z}$ are derived by permuting the indices as explained below. The final formulas are given by substituting the formulas for the derivatives of the Hermite basis functions, and exploiting their orthogonality properties. We find that

$$
\begin{aligned}
& B_{x}(\boldsymbol{x}, t) \int_{\Omega_{v}} v_{z} \frac{\partial f^{s}(\boldsymbol{x}, \boldsymbol{v}, t)}{\partial v_{y}} \psi^{n}\left(\xi_{x}^{s}\right) \psi^{m}\left(\xi_{y}^{s}\right) \psi^{p}\left(\xi_{z}^{s}\right) d \xi_{x}^{s} d \xi_{y}^{s} d \xi_{z}^{s} \\
& =-B_{x}(\boldsymbol{x}, t)\left[\frac{\alpha_{z}^{s}}{\alpha_{y}^{s}} \sqrt{m p} C_{n, m-1, p-1}(t)+\frac{\alpha_{z}^{s}}{\alpha_{y}^{s}} \sqrt{m(p+1)} C_{n, m-1, p+1}(t)+\frac{u_{z}^{s}}{\alpha_{y}^{s}} \sqrt{2 m} C_{n, m-1, p}(t)\right]
\end{aligned}
$$

and

$$
\begin{aligned}
& -B_{x}(\boldsymbol{x}, t) \int_{\Omega_{v}} v_{y} \frac{\partial f^{s}(\boldsymbol{x}, \boldsymbol{v}, t)}{\partial v_{z}} \psi^{n}\left(\xi_{x}^{s}\right) \psi^{m}\left(\xi_{y}^{s}\right) \psi^{p}\left(\xi_{z}^{s}\right) d \xi_{x}^{s} d \xi_{y}^{s} d \xi_{z}^{s} \\
& \quad=B_{x}(\boldsymbol{x}, t)\left[\frac{\alpha_{y}^{s}}{\alpha_{z}^{s}} \sqrt{m p} C_{n, m-1, p-1}+\frac{\alpha_{y}^{s}}{\alpha_{z}^{s}} \sqrt{(m+1) p} C_{n, m+1, p-1}+\sqrt{2 p} \frac{u_{y}^{s}}{\alpha_{z}^{s}} C_{n, m, p-1}\right] .
\end{aligned}
$$

We derive the two similar terms for $B_{y}$ through the permutation $(x, y, z) \rightarrow(y, z, x)$ and $(n, m, p) \rightarrow(m, p, n)$; and the two similar terms for $B_{z}$ through the permutation $(x, y, z) \rightarrow(z, x, y)$ and $(n, m, p) \rightarrow(p, n, m)$. 


\section{Appendix B. Proofs of conservation laws for the semi-discrete formulation}

To prove the conservation laws for the semi-discrete formulation, it is convenient to rewrite the HermiteDG numerical method in a variational form. To this end, we first introduce the following finite dimensional spaces:

$$
\begin{aligned}
& \mathcal{H}^{N}:=\operatorname{span}\left\{\Psi_{n, m, p}, \text { for }(n, m, p) \in\left\{(0,0,0), \ldots,\left(N_{v_{x}}, N_{v_{y}}, N_{v_{z}}\right)\right\}\right\} \\
& \widetilde{\mathcal{H}}^{N}:=\operatorname{span}\left\{\Psi^{n, m, p}, \text { for }(n, m, p) \in\left\{(0,0,0), \ldots,\left(N_{v_{x}}, N_{v_{y}}, N_{v_{z}}\right)\right\}\right\} \\
& \mathcal{V}^{N}:=\operatorname{span}\left\{\varphi^{I, l}, \text { for } I \equiv I_{i, j, k},(i, j, k) \in\left\{(1,1,1), \ldots\left(N_{x}, N_{y}, N_{z}\right)\right\}, l=1, \ldots, N_{l}\right\} .
\end{aligned}
$$

For any time $t \in[0, T]$, we assume that the numerical distribution function $f^{s, N}(\cdot, \cdot, t)$ defined in (22) belongs to $\mathcal{H}^{N} \times \mathcal{V}^{N}$ for any plasma species $s$. Similarly, we take the numerical electromagnetic fields $\boldsymbol{E}^{N}$ and $\boldsymbol{B}^{N}$ that are defined in (42) and (43) in the finite-dimensional space $\mathcal{V}^{N}$. Finally, for convenience of exposition, we report the DG approximations of the charge and current density introduced in (69) and (68)

$$
\begin{aligned}
\rho^{N}(\boldsymbol{x}, t) & =\sum_{s} \rho^{s, N}(\boldsymbol{x}, t)=\sum_{s} q^{s} \int_{\Omega_{v}} f^{s, N}(\boldsymbol{x}, \boldsymbol{v}, t) d \boldsymbol{v} \\
\boldsymbol{J}^{N}(\boldsymbol{x}, t) & =\sum_{s} \boldsymbol{J}^{s, N}(\boldsymbol{x}, t)=\sum_{s} q^{s} \int_{\Omega_{v}} \boldsymbol{v} f^{s, N}(\boldsymbol{x}, \boldsymbol{v}, t) d \boldsymbol{v} .
\end{aligned}
$$

The semi-discrete variational formulation of the Hermite-DG method reads as: For every species s, and any time $t \in[0, T]$ find $f^{s, N} \in \mathcal{H}^{N} \times \mathcal{V}^{N}$ and $\boldsymbol{E}^{N}, \boldsymbol{B}^{N} \in \mathcal{V}^{N}$ such that

$$
\begin{aligned}
A\left(\left(f^{s, N}, \boldsymbol{E}^{N}, \boldsymbol{B}^{N}\right),(\Psi, \varphi)\right) & =0 & & \forall(\Psi, \varphi) \in \widetilde{\mathcal{H}}^{N} \times \mathcal{V}^{N}, \\
B\left(\left(\boldsymbol{E}^{N}, \boldsymbol{B}^{N}\right), \varphi\right) & =L(\varphi) & & \forall \varphi \in \mathcal{V}^{N}, \\
f^{s, N}(\cdot, \cdot, 0) & =f_{0}^{s, N} & & \text { in } \Omega_{x} \times \Omega_{v}, \\
\boldsymbol{E}^{N}(\cdot, 0) & =\boldsymbol{E}_{0}^{N} & & \text { in } \Omega_{x}, \\
\boldsymbol{B}^{N}(\cdot, 0) & =\boldsymbol{B}_{0}^{N} & & \text { in } \Omega_{x},
\end{aligned}
$$

where $f_{0}^{s, N}, \boldsymbol{E}_{0}^{N}$ and $\boldsymbol{B}_{0}^{N}$ are the orthogonal projections of the initial conditions $f^{s}(\cdot, \cdot, 0), \boldsymbol{E}(\cdot, 0)$ and $\boldsymbol{B}(\cdot, 0)$ onto the spaces $\mathcal{H}^{N} \times \mathcal{V}^{N}, \mathcal{V}^{N}$ and $\mathcal{V}^{N}$, respectively.

To define the multilinear form $A$ in (B.4a), we first introduce the auxiliary vector function

$$
\boldsymbol{g}_{\Psi}^{s, N}(\boldsymbol{x}, t):=\int_{\Omega_{v}} \boldsymbol{v} f^{s, N}(\boldsymbol{x}, \boldsymbol{v}, t) \Psi(\boldsymbol{\xi}) d \boldsymbol{\xi}, \quad \forall \Psi \in \widetilde{\mathcal{H}}^{N},
$$

which explicitly depends on a given function in the dual Hermite space $\widetilde{\mathcal{H}}^{N}$. When $\Psi=\Psi^{n, m, p}$, we can optionally use the notation $\boldsymbol{g}_{n, m, p}^{s, N}$, which explicitly refers to the Hermite indices $n, m, p$.

Then, for any $f^{s, N} \in \mathcal{H}^{N} \times \mathcal{V}^{N}, \boldsymbol{E}^{N} \in \mathcal{V}^{N}, \boldsymbol{B}^{N} \in \mathcal{V}^{N}$, and $(\Psi, \varphi) \in \widetilde{\mathcal{H}}^{N} \times \mathcal{V}^{N}$, we define

$$
\begin{aligned}
A\left(\left(f^{s, N}, \boldsymbol{E}^{N}, \boldsymbol{B}^{N}\right),(\Psi, \varphi)\right):=\sum_{I}\left(\int_{I} \int_{\Omega_{v}} \frac{\partial f^{s, N}}{\partial t} \Psi(\boldsymbol{\xi}) d \boldsymbol{\xi} \varphi(\boldsymbol{x}) d \boldsymbol{x}-\int_{I} \boldsymbol{g}_{\Psi}^{s, N} \cdot \nabla_{\boldsymbol{x}} \varphi(\boldsymbol{x}) d \boldsymbol{x}\right. \\
\left.\quad+\int_{\partial I} \widehat{\boldsymbol{n} \cdot \boldsymbol{g}_{\Psi}^{s, N}} \varphi(\boldsymbol{x}) d S+\frac{q^{s}}{m^{s}} \frac{\omega_{c e}}{\omega_{p e}} \int_{I} \int_{\Omega_{v}}\left(\boldsymbol{E}^{N}+\boldsymbol{v} \times \boldsymbol{B}^{N}\right) \cdot \nabla_{\boldsymbol{v}} f^{s, N} \Psi(\boldsymbol{\xi}) d \boldsymbol{\xi} \varphi(\boldsymbol{x}) d \boldsymbol{x}\right) .
\end{aligned}
$$

The bilinear form associated with the discretization of Maxwell's equations is

$$
B\left(\left(\boldsymbol{E}^{N}, \boldsymbol{B}^{N}\right), \varphi\right):=B_{\boldsymbol{E}}\left(\left(\boldsymbol{E}^{N}, \boldsymbol{B}^{N}\right), \varphi\right)+B_{\boldsymbol{B}}\left(\left(\boldsymbol{E}^{N}, \boldsymbol{B}^{N}\right), \varphi\right) \quad \forall \boldsymbol{E}^{N}, \boldsymbol{B}^{N}, \varphi \in \mathcal{V}^{N}
$$


where, for $\boldsymbol{U}:=\left(\left(\boldsymbol{E}^{N}\right)^{T},\left(\boldsymbol{B}^{N}\right)^{T}\right)^{T}$ denoting the DG approximation of the vector-valued function $\boldsymbol{u}$ in (47),

$$
\begin{aligned}
& B_{\boldsymbol{E}}\left(\left(\boldsymbol{E}^{N}, \boldsymbol{B}^{N}\right), \varphi\right)=\sum_{I}\left(\int_{I} \frac{\partial \boldsymbol{E}^{N}}{\partial t} \varphi(\boldsymbol{x}) d \boldsymbol{x}-\int_{I} \mathbb{F}_{\boldsymbol{E}}(\boldsymbol{U}) \nabla_{\boldsymbol{x}} \varphi(\boldsymbol{x}) d \boldsymbol{x}+\int_{\partial I} \widehat{\mathbb{F}_{\boldsymbol{E}}(\boldsymbol{U}) \boldsymbol{n}} \varphi(\boldsymbol{x}) d S\right) \\
& B_{\boldsymbol{B}}\left(\left(\boldsymbol{E}^{N}, \boldsymbol{B}^{N}\right), \varphi\right)=\sum_{I}\left(\int_{I} \frac{\partial \boldsymbol{B}^{N}}{\partial t} \varphi(\boldsymbol{x}) d \boldsymbol{x}-\int_{I} \mathbb{F}_{\boldsymbol{B}}(\boldsymbol{U}) \nabla_{\boldsymbol{x}} \varphi(\boldsymbol{x}) d \boldsymbol{x}+\int_{\partial I} \widehat{\mathbb{F}_{\boldsymbol{B}}(\boldsymbol{U}) \boldsymbol{n}} \varphi(\boldsymbol{x}) d S\right) .
\end{aligned}
$$

The linear functional $L$ in (B.4b) reads

$$
L(\varphi):=-\frac{\omega_{p e}}{\omega_{c e}} \sum_{I} \int_{I} \boldsymbol{J}^{N} \varphi(\boldsymbol{x}) d \boldsymbol{x}, \quad \forall \varphi \in \mathcal{V}^{N} .
$$

The quantity $\widehat{\boldsymbol{n} \cdot \boldsymbol{g}_{\Psi}^{s, N}}$ in (B.6) and $\widehat{\mathbb{F}_{\boldsymbol{E}}(\boldsymbol{U}) \boldsymbol{n}}$ and $\widehat{\mathbb{F}_{\boldsymbol{B}}(\boldsymbol{U}) \boldsymbol{n}}$ in (B.8)-(B.9) are the numerical fluxes at the faces of the element boundaries. In the following analysis, we will consider the case of the central numerical flux, cf. (64), and the upwind numerical flux, cf. (65).

Remark B.1 The numerical flux function is uniquely defined at any cell interface up to the sign of the unit normal vector $\boldsymbol{n}$, which is conventionally oriented outwards with respect to cell $I$. The integral term on the cell boundary $\partial I$ can be split on the six faces that define cell I as follows:

$$
\int_{\partial I}=\left(\int_{\mathrm{f}_{i+\frac{1}{2}, j, k}}-\int_{\mathrm{f}_{i-\frac{1}{2}, j, k}}\right)+\left(\int_{\mathrm{f}_{i, j+\frac{1}{2}, k}}-\int_{\mathrm{f}_{i, j-\frac{1}{2}, k}}\right)+\left(\int_{\mathrm{f}_{i, j, k+\frac{1}{2}}}-\int_{\mathrm{f}_{i, j, k-\frac{1}{2}}}\right) .
$$

Summing over all the mesh cells provides three telescopic sums along the directions $x, y$, and $z$, respectively corresponding to the half-integer indices $i+\frac{1}{2}, j+\frac{1}{2}$, and $k+\frac{1}{2}$. For a periodic system in three spatial directions, the summation is zero. Therefore,

$$
\sum_{I} \int_{\partial I} \widehat{\mathbb{F}_{\boldsymbol{E}}(\boldsymbol{U}) \boldsymbol{n}} d S=\sum_{I} \int_{\partial I} \widehat{\mathbb{F}_{\boldsymbol{B}}(\boldsymbol{U}) \boldsymbol{n}} d S=0 .
$$

This remark will be used when proving the conservation of the number of particles and energy.

\section{B.1. Proof of Theorem 5.1 (conservation of the number of particles)}

The invariance in time of $\mathcal{N}^{\text {tot }}(t)$ and $\mathcal{N}^{s}(t)$ from the semi-discrete method can be shown by proving that the integral of $\partial f^{s, N} / \partial t$ on the phase space $\cup_{I \in \Omega_{x}} I \times \Omega_{v}$ is zero. Observe that $\varphi^{I, l}=1$ for $l=0$, and $\Psi^{n, m, p}=1$, for $n=m=p=0$. Then, we use (B.4a) and for each particle species $s$ we note that

$$
\begin{aligned}
\frac{d \mathcal{N}^{s}(t)}{d t}= & \sum_{I} \int_{I} \int_{\Omega_{v}} \frac{\partial f^{s, N}}{\partial t}(\boldsymbol{x}, \boldsymbol{v}, t) d \boldsymbol{v} d \boldsymbol{x}=\sum_{I} \int_{I}\left(\int_{\Omega_{v}} \frac{\partial f^{s, N}}{\partial t}(\boldsymbol{x}, \boldsymbol{v}, t) \Psi^{0,0,0}(\boldsymbol{\xi}) d \boldsymbol{v}\right) \varphi^{I, 0}(\boldsymbol{x}) d \boldsymbol{x} \\
= & \sum_{I} \int_{I}\left(\int_{\Omega_{v}} \boldsymbol{v} f^{s, N}(\boldsymbol{x}, \boldsymbol{v}, t) \Psi^{0,0,0}(\boldsymbol{\xi}) d \boldsymbol{\xi}\right) \cdot \nabla_{\boldsymbol{x}} \varphi^{I, 0}(\boldsymbol{x}) d \boldsymbol{x}-\sum_{I} \int_{\partial I} \widehat{\boldsymbol{n} \cdot \boldsymbol{g}_{0,0,0}^{s}} \varphi^{I, 0}(\boldsymbol{x}) d S \\
& -\frac{q^{s}}{m^{s}} \frac{\omega_{c e}}{\omega_{p e}} \sum_{I} \int_{I} \int_{\Omega_{v}}\left(\boldsymbol{E}^{N}+\boldsymbol{v} \times \boldsymbol{B}^{N}\right) \cdot \nabla_{\boldsymbol{v}} f^{s, N} \Psi^{0,0,0}(\boldsymbol{\xi}) d \boldsymbol{\xi} \varphi^{I, 0}(\boldsymbol{x}) d \boldsymbol{x}=: \mathrm{T}_{1}+\mathrm{T}_{2}+\mathrm{T}_{3} .
\end{aligned}
$$

Next, we prove that $\mathrm{T}_{1}=\mathrm{T}_{2}=\mathrm{T}_{3}=0$. The first term is zero because $\varphi^{I, 0} \equiv 1$ on every $I$. The second term $\mathrm{T}_{2}$ vanishes in view of Remark B.1. To see that the third term $\mathrm{T}_{3}$ is zero, we first transform the integral in $d \boldsymbol{\xi}$ as follows

$$
\begin{aligned}
\int_{\Omega_{v}}\left(\boldsymbol{E}^{N}+\boldsymbol{v} \times \boldsymbol{B}^{N}\right) \cdot \nabla_{\boldsymbol{v}} f^{s, N} \Psi^{0,0,0}(\boldsymbol{\xi}) d \boldsymbol{\xi}= & -\int_{\Omega_{v}} \nabla \cdot\left(\Psi^{0,0,0}(\boldsymbol{\xi})\left(\boldsymbol{E}^{N}+\boldsymbol{v} \times \boldsymbol{B}^{N}\right)\right) f^{s, N} d \boldsymbol{\xi} \\
& +[\text { zero boundary terms for }|\boldsymbol{\xi}| \rightarrow \pm \infty],
\end{aligned}
$$


by an integration by parts. We recall that the boundary terms are zero because $f^{s} \rightarrow 0$ exponentially for $|\boldsymbol{\xi}| \rightarrow \pm \infty$. The right-hand side of (B.11) is zero because $\Psi^{0,0,0}=1$ and $\nabla \cdot\left(\boldsymbol{E}^{N}+\boldsymbol{v} \times \boldsymbol{B}^{N}\right)=0$, thus implying that $\mathrm{T}_{3}=0$.

\section{B.2. Proof of Theorem 5.2 (conservation of total momentum)}

To derive an evolution equation for the vector-valued momentum $\boldsymbol{P}_{f}^{N}(t)$ defined in (71), we exploit the fact that each component of the velocity field $\boldsymbol{v}$ belongs to $\widetilde{\mathcal{H}}^{N}$. Therefore, for every cell $I$ and every vector component $\beta \in\{x, y, z\}$ we consider the semi-discrete Vlasov equation (B.4a) with $\Psi(\boldsymbol{\xi}) \equiv v_{\beta}$ and $\varphi=\varphi^{I, 0}=1$. We change the integration variable from $\boldsymbol{\xi}$ to $\boldsymbol{v}$ in all terms, we multiply by the species mass $m^{s}$ and we sum over the species index. Since $\nabla_{\boldsymbol{x}} \varphi^{I, 0}(\boldsymbol{x})=0$, we find that

$$
\begin{aligned}
\frac{d \boldsymbol{P}_{f}^{N}}{d t} & =\sum_{s} m^{s} \int_{I} \int_{\Omega_{v}} \frac{\partial f^{s, N}}{\partial t} \boldsymbol{v} d \boldsymbol{v} d \boldsymbol{x} \\
& =-\sum_{s} m^{s} \int_{\partial I} \widehat{\boldsymbol{n} \cdot \boldsymbol{g}_{\boldsymbol{v}}^{s, N}} d S-\sum_{s} q^{s} \frac{\omega_{c e}}{\omega_{p e}} \int_{I} \int_{\Omega_{v}}\left(\boldsymbol{E}^{N}+\boldsymbol{v} \times \boldsymbol{B}^{N}\right) \cdot \nabla_{\boldsymbol{v}} f^{s, N} \boldsymbol{v} d \boldsymbol{v} d \boldsymbol{x},
\end{aligned}
$$

where $\widehat{\boldsymbol{n} \cdot \boldsymbol{g}_{\boldsymbol{v}}^{s, N}}$ is the numerical flux of

$$
\boldsymbol{n} \cdot \boldsymbol{g}_{\boldsymbol{v}}^{s, N}(\boldsymbol{x}, t):=\int_{\Omega_{v}}(\boldsymbol{n} \cdot \boldsymbol{v}) \boldsymbol{v} f^{s, N}(\boldsymbol{x}, \boldsymbol{v}, t) d \boldsymbol{v} .
$$

Equation (B.12) holds at any time $t$, for every element $I$. We integrate by parts the last term of (B.12), and we find that

$$
\frac{d \boldsymbol{P}_{f}^{N}}{d t}=-\sum_{s} m^{s} \int_{\partial I} \widehat{\boldsymbol{n} \cdot \boldsymbol{g}_{\boldsymbol{v}}^{s, N}} d S+\sum_{s} q^{s} \frac{\omega_{c e}}{\omega_{p e}} \int_{I} \int_{\Omega_{v}}\left(\boldsymbol{E}^{N}+\boldsymbol{v} \times \boldsymbol{B}^{N}\right) f^{s, N} d \boldsymbol{v} d \boldsymbol{x},
$$

since $\nabla_{\boldsymbol{v}} \cdot\left(\boldsymbol{E}^{N}+\boldsymbol{v} \times \boldsymbol{B}^{N}\right)=0$ and $\nabla_{\boldsymbol{v}} \boldsymbol{v}$ is the identity matrix. Using definitions (B.2) and (B.3), the last term of (B.14) can be rewritten as

$$
\begin{aligned}
\sum_{s} q^{s} \frac{\omega_{c e}}{\omega_{p e}} & \int_{I} \int_{\Omega_{v}}\left(\boldsymbol{E}^{N}+\boldsymbol{v} \times \boldsymbol{B}^{N}\right) f^{s, N} d \boldsymbol{v} d \boldsymbol{x} \\
& =\frac{\omega_{c e}}{\omega_{p e}}\left(\int_{I}\left(\sum_{s} q^{s} \int_{\Omega_{v}} f^{s, N} d \boldsymbol{v}\right) \boldsymbol{E}^{N} d \boldsymbol{x}+\int_{I}\left(\sum_{s} q^{s} \int_{\Omega_{v}} \boldsymbol{v} f^{s, N} d \boldsymbol{v}\right) \times \boldsymbol{B}^{N} d \boldsymbol{x}\right) \\
& =\frac{\omega_{c e}}{\omega_{p e}} \int_{I} \rho^{N} \boldsymbol{E}^{N} d \boldsymbol{x}+\frac{\omega_{c e}}{\omega_{p e}} \int_{I} \boldsymbol{J}^{N} \times \boldsymbol{B}^{N} d \boldsymbol{x} .
\end{aligned}
$$

Using (B.15) in (B.14) we find that

$$
\sum_{s} m^{s} \int_{I} \int_{\Omega_{v}} \frac{\partial f^{s, N}}{\partial t} \boldsymbol{v} d \boldsymbol{v} d \boldsymbol{x}+\sum_{s} m^{s} \int_{\partial I} \widehat{\boldsymbol{n} \cdot \boldsymbol{g}_{\boldsymbol{v}}^{s, N}} d S-\frac{\omega_{c e}}{\omega_{p e}} \int_{I}\left(\rho^{N} \boldsymbol{E}^{N}+\boldsymbol{J}^{N} \times \boldsymbol{B}^{N}\right) d \boldsymbol{x}=0 .
$$

Then, we integrate by parts equations (B.8) and (B.9) with $\varphi=\varphi^{I, l}$, we use definition (48) to obtain

$$
\begin{aligned}
& \int_{I} \frac{\partial \boldsymbol{E}^{N}}{\partial t} \varphi^{I, l} d \boldsymbol{x}-\int_{I}\left(\nabla_{\boldsymbol{x}} \times \boldsymbol{B}^{N}\right) \varphi^{I, l} d \boldsymbol{x}+\int_{\partial I}\left(\widehat{\mathbb{F}_{\boldsymbol{E}}(\boldsymbol{U}) \boldsymbol{n}}-\mathbb{F}_{\boldsymbol{E}}(\boldsymbol{U}) \boldsymbol{n}\right) \varphi^{I, l} d S+\frac{\omega_{p e}}{\omega_{c e}} \int_{I} \boldsymbol{J}^{N} \varphi^{I, l} d \boldsymbol{x}=0 \\
& \int_{I} \frac{\partial \boldsymbol{B}^{N}}{\partial t} \varphi^{I, l} d \boldsymbol{x}+\int_{I}\left(\nabla_{\boldsymbol{x}} \times \boldsymbol{E}^{N}\right) \varphi^{I, l} d \boldsymbol{x}+\int_{\partial I}\left(\widehat{\mathbb{F}_{\boldsymbol{B}}(\boldsymbol{U}) \boldsymbol{n}}-\mathbb{F}_{\boldsymbol{B}}(\boldsymbol{U}) \boldsymbol{n}\right) \varphi^{I, l} d S=0 .
\end{aligned}
$$


We take the cross product of equation (B.17) and $\boldsymbol{B}^{I, l}(t)$, and the cross product of equation (B.18) and $\boldsymbol{E}^{I, l}(t)$, we sum over $l$, and in view of the expansions (42) and (43), we obtain the two equations:

$$
\begin{aligned}
& \int_{I} \frac{\partial \boldsymbol{E}^{N}}{\partial t} \times \boldsymbol{B}^{N} d \boldsymbol{x}-\int_{I}\left(\nabla_{\boldsymbol{x}} \times \boldsymbol{B}^{N}\right) \times \boldsymbol{B}^{N} d \boldsymbol{x}+\int_{\partial I}\left(\widehat{\mathbb{F}_{\boldsymbol{E}}(\boldsymbol{U}) \boldsymbol{n}}-\mathbb{F}_{\boldsymbol{E}}(\boldsymbol{U}) \boldsymbol{n}\right) \times \boldsymbol{B}^{N} d S \\
& \quad+\frac{\omega_{p e}}{\omega_{c e}} \int_{I} \boldsymbol{J}^{N} \times \boldsymbol{B}^{N} d \boldsymbol{x}=0, \\
& \int_{I} \frac{\partial \boldsymbol{B}^{N}}{\partial t} \times \boldsymbol{E}^{N} d \boldsymbol{x}+\int_{I}\left(\nabla_{\boldsymbol{x}} \times \boldsymbol{E}^{N}\right) \times \boldsymbol{E}^{N} d \boldsymbol{x}+\int_{\partial I}\left(\widehat{\mathbb{F}_{\boldsymbol{B}}(\boldsymbol{U}) \boldsymbol{n}}-\mathbb{F}_{\boldsymbol{B}}(\boldsymbol{U}) \boldsymbol{n}\right) \times \boldsymbol{E}^{N} d S=0 .
\end{aligned}
$$

We subtract (B.20) from (B.19) and we find that

$$
\begin{aligned}
\frac{d}{d t} \int_{I}\left(\boldsymbol{E}^{N} \times \boldsymbol{B}^{N}\right) d \boldsymbol{x}= & -\int_{I}\left(\boldsymbol{B}^{N} \times\left(\nabla_{\boldsymbol{x}} \times \boldsymbol{B}^{N}\right)+\boldsymbol{E}^{N} \times\left(\nabla_{\boldsymbol{x}} \times \boldsymbol{E}^{N}\right)\right) d \boldsymbol{x} \\
& -\int_{\partial I} \mathcal{B}_{I} d S-\frac{\omega_{p e}}{\omega_{c e}} \int_{I} \boldsymbol{J}^{N} \times \boldsymbol{B}^{N} d \boldsymbol{x},
\end{aligned}
$$

where

$$
\mathcal{B}_{I}=\left(\widehat{\mathbb{F}_{\boldsymbol{E}}(\boldsymbol{U}) \boldsymbol{n}}-\mathbb{F}_{\boldsymbol{E}}(\boldsymbol{U}) \boldsymbol{n}\right) \times \boldsymbol{B}^{N}-\left(\widehat{\mathbb{F}_{\boldsymbol{B}}(\boldsymbol{U}) \boldsymbol{n}}-\mathbb{F}_{\boldsymbol{B}}(\boldsymbol{U}) \boldsymbol{n}\right) \times \boldsymbol{E}^{N}
$$

Since $-\mathbb{F}_{\boldsymbol{E}}(\boldsymbol{U}) \boldsymbol{n} \times \boldsymbol{B}^{N}=\boldsymbol{n} \times \boldsymbol{B}^{N} \times \boldsymbol{B}^{N}=0$ and $\mathbb{F}_{\boldsymbol{B}}(\boldsymbol{U}) \boldsymbol{n} \times \boldsymbol{E}^{N}=\boldsymbol{n} \times \boldsymbol{E}^{N} \times \boldsymbol{E}^{N}=0$, the term $\mathcal{B}_{I}$ takes the form given in the statement of Theorem 5.2. Finally, the assertion of the theorem follows by summing (B.21) to (B.16).

\section{B.3. Proof of Theorem 5.3 (conservation of total energy)}

We split the proof of the theorem in three steps. To ease the notation, we drop the explicit dependence on $\boldsymbol{x}, \boldsymbol{v}$ and $t$ of the fields $f^{s, N}, \boldsymbol{E}^{N}, \boldsymbol{B}^{N}$, and $\boldsymbol{J}^{N}$.

(i) In the first step, we prove that the kinetic energy satisfies

$$
\frac{d \mathcal{E}_{\text {kin }}^{N}(t)}{d t}:=\frac{1}{2} \sum_{s} m^{s} \sum_{I} \int_{I}\left(\int_{\Omega_{v}} \frac{\partial f^{s, N}}{\partial t}|\boldsymbol{v}|^{2} d \boldsymbol{v}\right) d \boldsymbol{x}=\frac{\omega_{c e}}{\omega_{p e}} \sum_{I} \int_{I} \boldsymbol{E}^{N} \cdot \boldsymbol{J}^{N} d \boldsymbol{x}
$$

where $\boldsymbol{J}^{N}$ is the approximate current density defined in (B.3).

(ii) In the second step, we prove that

$$
\frac{d \mathcal{E}_{\boldsymbol{E}, \boldsymbol{B}}^{N}(t)}{d t}:=\frac{1}{2}\left(\frac{\omega_{c e}}{\omega_{p e}}\right)^{2} \sum_{I} \frac{d}{d t} \int_{I}\left(\left|\boldsymbol{E}^{N}\right|^{2}+\left|\boldsymbol{B}^{N}\right|^{2}\right) d \boldsymbol{x}=-\frac{\omega_{c e}}{\omega_{p e}} \sum_{I} \int_{I} \boldsymbol{E}^{N} \cdot \boldsymbol{J}^{N} d \boldsymbol{x}+\left(\frac{\omega_{c e}}{\omega_{p e}}\right)^{2} \widetilde{\Phi},
$$

where

$$
\widetilde{\Phi}:=\sum_{I}\left(\int_{\partial I}\left(\mathbb{F}_{\boldsymbol{E}} \boldsymbol{n}-\widehat{\mathbb{F}_{\boldsymbol{E}} \boldsymbol{n}}\right) \cdot \boldsymbol{E}^{N} d S+\int_{\partial I}\left(\mathbb{F}_{\boldsymbol{B}} \boldsymbol{n}-\widehat{\mathbb{F}_{\boldsymbol{B}} \boldsymbol{n}}\right) \cdot \boldsymbol{B}^{N} d S-\int_{\partial I} \boldsymbol{n} \cdot\left(\boldsymbol{E}^{N} \times \boldsymbol{B}^{N}\right) d S\right) .
$$

(iii) In the third step, we prove that

$$
\widetilde{\Phi}=-\sum_{\mathrm{f}} \mathcal{J}_{\mathrm{f}} \leq 0
$$

where $\mathcal{J}_{\mathrm{f}}$ is defined in $(82)$.

The assertion of the theorem follows by substituting (B.24) in (B.23), and then using definition (B.25) and the jump relation (B.26). 
Proof of (B.23). Since $|\boldsymbol{v}|^{2}$ is a linear combination of the Hermite polynomials $\boldsymbol{\Psi}^{n, m, p}(\boldsymbol{\xi})$ for $n+m+p \leq 2$, it belongs to the velocity approximation space. Therefore, the evolution of the kinetic energy can be computed using equation (B.4a) (with $l=0$ ):

$$
\begin{aligned}
& \frac{d \mathcal{E}_{\text {kin }}^{N}(t)}{d t}=\frac{1}{2} \sum_{s} m^{s} \sum_{I} \int_{I} \int_{\Omega_{v}} \frac{\partial f^{s, N}}{\partial t}|\boldsymbol{v}|^{2} d \boldsymbol{v} d \boldsymbol{x} \\
& =-\frac{1}{2} \sum_{s} m^{s} \sum_{I} \int_{\partial I} \widehat{\boldsymbol{n} \cdot \boldsymbol{g}^{s, N}} d S-\frac{1}{2} \sum_{s} m^{s} \sum_{I} \int_{I} \frac{q^{s}}{m^{s}} \frac{\omega_{c e}}{\omega_{p e}} \int_{\Omega_{v}}\left(\boldsymbol{E}^{N}+\boldsymbol{v} \times \boldsymbol{B}^{N}\right) \cdot \nabla_{\boldsymbol{v}} f^{s, N}|\boldsymbol{v}|^{2} d \boldsymbol{v} d \boldsymbol{x} \\
& =: \mathrm{T}_{1}+\mathrm{T}_{2},
\end{aligned}
$$

where $\widehat{\boldsymbol{n} \cdot \boldsymbol{g}^{s, N}}$ is the numerical flux at the cell interfaces $\partial I$ of the vector field

$$
\boldsymbol{g}^{s, N}(\boldsymbol{x}, t):=\int_{\Omega_{v}} \boldsymbol{v} f^{s, N}(\boldsymbol{x}, \boldsymbol{v}, t)|\boldsymbol{v}|^{2} d \boldsymbol{v} .
$$

Term $\mathrm{T}_{1}$ is zero because of Remark (B.1). To compute the term $\mathrm{T}_{2}$ in (B.27), we integrate by parts with respect to $\boldsymbol{v}$ so that,

$$
\begin{aligned}
\int_{\Omega_{v}} & \left(\boldsymbol{E}^{N}+\boldsymbol{v} \times \boldsymbol{B}^{N}\right) \cdot \nabla_{\boldsymbol{v}} f^{s, N}|\boldsymbol{v}|^{2} d \boldsymbol{v} \\
& =-\int_{\Omega_{v}} \nabla_{\boldsymbol{v}} \cdot\left(|\boldsymbol{v}|^{2}\left(\boldsymbol{E}^{N}+\boldsymbol{v} \times \boldsymbol{B}^{N}\right)\right) f^{s, N} d \boldsymbol{v}+[\text { zero boundary terms for }|\boldsymbol{v}| \rightarrow \infty] \\
& =-2 \boldsymbol{E}^{N} \cdot \int_{\Omega_{v}} \boldsymbol{v} f^{s, N} d \boldsymbol{v},
\end{aligned}
$$

since $\nabla_{\boldsymbol{v}}|\boldsymbol{v}|^{2}=2 \boldsymbol{v}$ and $\nabla_{\boldsymbol{v}} \cdot\left(\boldsymbol{E}^{N}+\boldsymbol{v} \times \boldsymbol{B}^{N}\right)=0$. According to the definition of $\mathrm{T}_{2}$ from (B.27) and using the definition of $\boldsymbol{J}^{N}$ in (B.3), it holds that

$$
\begin{aligned}
\mathrm{T}_{2} & =-\frac{1}{2} \sum_{s} q^{s} \frac{\omega_{c e}}{\omega_{p e}} \sum_{I} \int_{I} \int_{\Omega_{v}}\left(\boldsymbol{E}^{N}+\boldsymbol{v} \times \boldsymbol{B}^{N}\right) \cdot \nabla_{\boldsymbol{v}} f^{s, N}|\boldsymbol{v}|^{2} d \boldsymbol{v} d \boldsymbol{x} \\
& =\sum_{s} q^{s} \frac{\omega_{c e}}{\omega_{p e}} \sum_{I} \int_{I} \boldsymbol{E}^{N} \cdot\left(\int_{\Omega_{v}} \boldsymbol{v} f^{s, N} d \boldsymbol{v}\right) d \boldsymbol{x}=\frac{\omega_{c e}}{\omega_{p e}} \sum_{I} \int_{I} \boldsymbol{E}^{N} \cdot \boldsymbol{J}^{N} d \boldsymbol{x} .
\end{aligned}
$$

Equation (B.23) follows by substituting in (B.27), $\mathrm{T}_{1}=0$ and the expression of $\mathrm{T}_{2}$ from (B.30).

Proof of (B.24). We take the Euclidean product of (B.8) with $\boldsymbol{E}^{I, l}$ and sum over $l=1, \ldots N_{l}$,

$$
\begin{aligned}
\sum_{l=1}^{N_{l}}\left(\int_{I} \frac{\partial \boldsymbol{E}^{N}}{\partial t} \cdot \boldsymbol{E}^{I, l} \varphi^{I, l} d \boldsymbol{x}-\int_{I}\left(\mathbb{F}_{\boldsymbol{E}}(\boldsymbol{U}) \nabla_{\boldsymbol{x}} \varphi^{I, l}\right) \cdot \boldsymbol{E}^{I, l} d \boldsymbol{x}\right. \\
\left.\quad+\int_{\partial I} \widehat{\mathbb{F}_{\boldsymbol{E}}(\boldsymbol{U}) \boldsymbol{n}} \cdot \boldsymbol{E}^{I, l} \varphi^{I, l} d \boldsymbol{x}+\frac{\omega_{p e}}{\omega_{c e}} \int_{I} \boldsymbol{J}^{N} \cdot \boldsymbol{E}^{I, l} \varphi^{I, l} d \boldsymbol{x}\right)=0
\end{aligned}
$$

Integrating by parts yields

$$
\int_{I} \frac{\partial \boldsymbol{E}^{N}}{\partial t} \cdot \boldsymbol{E}^{N} d \boldsymbol{x}+\frac{\omega_{p e}}{\omega_{c e}} \int_{I} \boldsymbol{E}^{N} \cdot \boldsymbol{J}^{N} d \boldsymbol{x}=-\int_{I}\left(\nabla_{\boldsymbol{x}} \cdot \mathbb{F}_{\boldsymbol{E}}(\boldsymbol{U})\right) \cdot \boldsymbol{E}^{N} d \boldsymbol{x}+\int_{\partial I}\left(\mathbb{F}_{\boldsymbol{E}} \boldsymbol{n}-\widehat{\mathbb{F}_{\boldsymbol{E}} \boldsymbol{n}}\right) \cdot \boldsymbol{E}^{N} d S .
$$

Then, we use formula (48) to express the divergence of the flux,

$$
\frac{d}{d t} \frac{1}{2} \int_{I}\left|\boldsymbol{E}^{N}\right|^{2} d \boldsymbol{x}+\frac{\omega_{p e}}{\omega_{c e}} \int_{I} \boldsymbol{E}^{N} \cdot \boldsymbol{J}^{N} d \boldsymbol{x}=\int_{I} \nabla_{\boldsymbol{x}} \times \boldsymbol{B}^{N} \cdot \boldsymbol{E}^{N} d \boldsymbol{x}+\int_{\partial I}\left(\mathbb{F}_{\boldsymbol{E}} \boldsymbol{n}-\widehat{\mathbb{F}_{\boldsymbol{E}} \boldsymbol{n}}\right) \cdot \boldsymbol{E}^{N} d S
$$

Similarly, we multiply equation (B.9) by $\boldsymbol{B}^{I, l}$, sum over $l=1, \ldots N_{l}$, integrate by parts, and use formula (48),

$$
\int_{I} \frac{\partial \boldsymbol{B}^{N}}{\partial t} \cdot \boldsymbol{B}^{N} d \boldsymbol{x}=-\int_{I}\left(\nabla_{\boldsymbol{x}} \times \boldsymbol{E}^{N}\right) \cdot \boldsymbol{B}^{N} d \boldsymbol{x}+\int_{\partial I}\left(\mathbb{F}_{\boldsymbol{B}} \boldsymbol{n}-\widehat{\mathbb{F}_{\boldsymbol{B}} \boldsymbol{n}}\right) \cdot \boldsymbol{B}^{N} d S
$$


Summing (B.31) and (B.32) we obtain,

$$
\frac{d}{d t} \frac{1}{2} \int_{I}\left(\left|\boldsymbol{E}^{N}\right|^{2}+\left|\boldsymbol{B}^{N}\right|^{2}\right) d \boldsymbol{x}+\frac{\omega_{p e}}{\omega_{c e}} \int_{I} \boldsymbol{E}^{N} \cdot \boldsymbol{J}^{N} d \boldsymbol{x}=\int_{I}\left(\nabla_{\boldsymbol{x}} \times \boldsymbol{B}^{N} \cdot \boldsymbol{E}^{N}-\nabla_{\boldsymbol{x}} \times \boldsymbol{E}^{N} \cdot \boldsymbol{B}^{N}\right) d \boldsymbol{x}+\widetilde{\Phi}^{I},
$$

where $\widetilde{\Phi}^{I}$ is defined as

$$
\widetilde{\Phi}^{I}:=\int_{\partial I}\left(\mathbb{F}_{\boldsymbol{E}} \boldsymbol{n}-\widehat{\mathbb{F}_{\boldsymbol{E}} \boldsymbol{n}}\right) \cdot \boldsymbol{E}^{N} d S+\int_{\partial I}\left(\mathbb{F}_{\boldsymbol{B}} \boldsymbol{n}-\widehat{\mathbb{F}_{\boldsymbol{B}} \boldsymbol{n}}\right) \cdot \boldsymbol{B}^{N} d S
$$

Observe that

$$
\nabla_{\boldsymbol{x}} \times \boldsymbol{B}^{N} \cdot \boldsymbol{E}^{N}-\nabla_{\boldsymbol{x}} \times \boldsymbol{E}^{N} \cdot \boldsymbol{B}^{N}=-\nabla_{\boldsymbol{x}} \cdot\left(\boldsymbol{E}^{N} \times \boldsymbol{B}^{N}\right),
$$

so that (B.33) becomes

$$
\frac{d}{d t} \frac{1}{2} \int_{I}\left(\left|\boldsymbol{E}^{N}\right|^{2}+\left|\boldsymbol{B}^{N}\right|^{2}\right) d \boldsymbol{x}+\frac{\omega_{p e}}{\omega_{c e}} \int_{I} \boldsymbol{E}^{N} \cdot \boldsymbol{J}^{N} d \boldsymbol{x}=-\int_{I} \nabla_{\boldsymbol{x}} \cdot\left(\boldsymbol{E}^{N} \times \boldsymbol{B}^{N}\right) d \boldsymbol{x}+\widetilde{\Phi}^{I} .
$$

Using the divergence theorem and summing over the mesh elements $I \subset \Omega_{x}$ yields,

$$
\frac{d}{d t} \frac{1}{2} \sum_{I} \int_{I}\left(\left|\boldsymbol{E}^{N}\right|^{2}+\left|\boldsymbol{B}^{N}\right|^{2}\right) d \boldsymbol{x}+\frac{\omega_{p e}}{\omega_{c e}} \sum_{I} \int_{I} \boldsymbol{E}^{N} \cdot \boldsymbol{J}^{N} d \boldsymbol{x}=\sum_{I}\left(\widetilde{\Phi}^{I}-\int_{\partial I} \boldsymbol{n} \cdot\left(\boldsymbol{E}^{N} \times \boldsymbol{B}^{N}\right) d \boldsymbol{x}\right)=: \widetilde{\Phi}
$$

which gives (B.24).

Proof of(B.26). Let us consider the term

$$
\widetilde{\Phi}=\sum_{I}\left(\int_{\partial I}\left(\mathbb{F}_{\boldsymbol{E}} \boldsymbol{n}-\widehat{\mathbb{F}_{\boldsymbol{E}} \boldsymbol{n}}\right) \cdot \boldsymbol{E}^{N} d S+\int_{\partial I}\left(\mathbb{F}_{\boldsymbol{B}} \boldsymbol{n}-\widehat{\mathbb{F}_{\boldsymbol{B}} \boldsymbol{n}}\right) \cdot \boldsymbol{B}^{N} d S-\int_{\partial I} \boldsymbol{n} \cdot\left(\boldsymbol{E}^{N} \times \boldsymbol{B}^{N}\right) d \boldsymbol{x}\right) .
$$

Note that, by the vector identity (54), it holds

$$
\boldsymbol{n} \cdot\left(\boldsymbol{E}^{N} \times \boldsymbol{B}^{N}\right)=\frac{1}{2}\left(\mathbb{F}_{\boldsymbol{E}} \boldsymbol{n} \cdot \boldsymbol{E}^{N}+\mathbb{F}_{\boldsymbol{B}} \boldsymbol{n} \cdot \boldsymbol{B}^{N}\right) .
$$

Therefore,

$$
-\boldsymbol{n} \cdot\left(\boldsymbol{E}^{N} \times \boldsymbol{B}^{N}\right)+\left(\mathbb{F}_{\boldsymbol{E}} \boldsymbol{n} \cdot \boldsymbol{E}^{N}+\mathbb{F}_{\boldsymbol{B}} \boldsymbol{n} \cdot \boldsymbol{B}^{N}\right)=\frac{1}{2}\left(\mathbb{F}_{\boldsymbol{E}} \boldsymbol{n} \cdot \boldsymbol{E}^{N}+\mathbb{F}_{\boldsymbol{B}} \boldsymbol{n} \cdot \boldsymbol{B}^{N}\right),
$$

and

$$
\widetilde{\Phi}=\sum_{I} \int_{\partial I}\left(\frac{1}{2}\left(\mathbb{F}_{\boldsymbol{E}} \boldsymbol{n} \cdot \boldsymbol{E}^{N}+\mathbb{F}_{\boldsymbol{B}} \boldsymbol{n} \cdot \boldsymbol{B}^{N}\right)-\left(\widehat{\mathbb{F}_{\boldsymbol{E}} \boldsymbol{n}} \cdot \boldsymbol{E}^{N}+\widehat{\mathbb{F}_{\boldsymbol{B}} \boldsymbol{n}} \cdot \boldsymbol{B}^{N}\right)\right) d S
$$

Moreover, it holds

$$
\mathbb{F}(\boldsymbol{U}) \boldsymbol{n} \cdot \boldsymbol{U}=\mathbb{F}_{\boldsymbol{E}} \boldsymbol{n} \cdot \boldsymbol{E}^{N}+\mathbb{F}_{\boldsymbol{B}} \boldsymbol{n} \cdot \boldsymbol{B}^{N}, \quad \widehat{\mathbb{F}(\boldsymbol{U}) \boldsymbol{n}} \cdot \boldsymbol{U}=\widehat{\mathbb{F}_{\boldsymbol{E}} \boldsymbol{n}} \cdot \boldsymbol{E}^{N}+\widehat{\mathbb{F}_{\boldsymbol{B}} \boldsymbol{n}} \cdot \boldsymbol{B}^{N},
$$

where the flux $\mathbb{F}(\boldsymbol{U})$ is given by (48). Moreover, we can reformulate the summation on the boundary cells as a summation on the cell interfaces by denoting the quantities referring to the two opposite sides with the superscripts \pm , and rewrite (B.37) as

$$
\widetilde{\Phi}=\sum_{I} \int_{\partial I}\left(\frac{1}{2} \mathbb{F}(\boldsymbol{U}) \boldsymbol{n} \cdot \boldsymbol{U}-\widehat{\mathbb{F}(\boldsymbol{U}) \boldsymbol{n}} \cdot \boldsymbol{U}\right) d S=\sum_{\mathrm{f}} \int_{\mathrm{f}} \mathcal{F}_{\mathrm{f}}\left(\boldsymbol{U}_{\mathrm{f}}^{+}, \boldsymbol{U}_{\mathrm{f}}^{-}\right) d S,
$$

where

$$
\mathcal{F}_{\mathrm{f}}\left(\boldsymbol{U}_{\mathrm{f}}^{+}, \boldsymbol{U}_{\mathrm{f}}^{-}\right):=\frac{1}{2}\left(\mathbb{F}\left(\boldsymbol{U}_{\mathrm{f}}^{+}\right) \boldsymbol{n}_{\mathrm{f}}^{-} \cdot \boldsymbol{U}_{\mathrm{f}}^{+}+\mathbb{F}\left(\boldsymbol{U}_{\mathrm{f}}^{-}\right) \boldsymbol{n}_{\mathrm{f}}^{+} \cdot \boldsymbol{U}_{\mathrm{f}}^{-}\right)-\left(\widehat{\mathbb{F}\left(\boldsymbol{U}_{\mathrm{f}}\right) \boldsymbol{n}_{\mathrm{f}}^{-}} \cdot \boldsymbol{U}_{\mathrm{f}}^{+}+\widehat{\mathbb{F}\left(\boldsymbol{U}_{\mathrm{f}}\right) \boldsymbol{n}_{\mathrm{f}}^{+}} \cdot \boldsymbol{U}_{\mathrm{f}}^{-}\right),
$$

at any given mesh face $\mathbf{f}$. Now, let $\mathbf{f}_{\beta}$ be a mesh face orthogonal to the direction $\beta$, for $\beta \in\{x, y, z\}$. Then, the $\beta$-th components of the unit vectors orthogonal to $\mathrm{f}_{\beta}$ are $n_{\beta}^{+}=-n_{\beta}^{-}=+1$, while the other components are zero. We refer to Figure 1 for a schematic representation of the quantities involved in the definition of the 
numerical fluxes. We recall that for a generic face $\mathrm{f}$ we use the notation $\mathbb{F}\left(\boldsymbol{U}_{\mathrm{f}}\right) \boldsymbol{n}_{\mathrm{f}} \cdot \boldsymbol{U}_{\mathrm{f}}=\sum_{\beta \in\{x, y, z\}} n_{\beta} \mathbb{F}_{\beta} \boldsymbol{U}_{\mathrm{f}} \cdot \boldsymbol{U}_{\mathrm{f}}$, where the matrices $\mathbb{F}_{\beta} \in \mathbb{R}^{6 \times 6}$ are defined in Section 4. If the last term is interpreted as the usual matrixvector product, we can write that $\mathbb{F}\left(\boldsymbol{U}_{\mathrm{f}}\right) \boldsymbol{n}_{\mathrm{f}} \cdot \boldsymbol{U}_{\mathrm{f}}=\boldsymbol{U}_{\mathrm{f}}^{\top}\left(\sum_{\beta \in\{x, y, z\}} n_{\beta} \mathbb{F}_{\beta}\right) \boldsymbol{U}_{\mathrm{f}}$. For each direction $\beta$, we denote $\mathbb{F}_{\beta}^{ \pm}=\mathbb{F}^{ \pm} n^{ \pm}=\sum_{\beta} n_{\beta}^{ \pm} \mathbb{F}_{\beta}^{ \pm}= \pm \mathbb{F}_{\beta}^{ \pm}$, and we recall that $\mathbb{F}_{\beta}=\mathbb{F}_{\beta}^{+}+\mathbb{F}_{\beta}^{-}$and $\left|\mathbb{F}_{\beta}\right|=\mathbb{F}_{\beta}^{+}-\mathbb{F}_{\beta}^{-}$. A straightforward calculation yields:

$$
\begin{aligned}
\mathcal{F}_{\mathrm{f}_{\beta}}\left(\boldsymbol{U}_{\mathrm{f}_{\beta}}^{+}, \boldsymbol{U}_{\mathrm{f}_{\beta}}^{-}\right) & =\frac{1}{2}\left(\left(\boldsymbol{U}_{\mathrm{f}_{\beta}}^{-}\right)^{T} \mathbb{F}_{\beta} \boldsymbol{U}_{\mathrm{f}_{\beta}}^{-}-\left(\boldsymbol{U}_{\mathrm{f}_{\beta}}^{+}\right)^{T} \mathbb{F}_{\beta} \boldsymbol{U}_{\mathrm{f}_{\beta}}^{+}\right)-\left(\boldsymbol{U}_{\mathrm{f}_{\beta}}^{-}-\boldsymbol{U}_{\mathrm{f}_{\beta}}^{+}\right)^{T} \widehat{\mathbb{F}_{\beta}(\boldsymbol{U}) \boldsymbol{n}_{\mathrm{f}_{\beta}}} \\
& =-\left(\boldsymbol{U}_{\mathrm{f}_{\beta}}^{+}-\boldsymbol{U}_{\mathrm{f}_{\beta}}^{-}\right)^{T}\left(\mathbb{F}_{\beta} \frac{\left(\boldsymbol{U}_{\mathrm{f}_{\beta}}^{+}+\boldsymbol{U}_{\mathrm{f}_{\beta}}^{-}\right)}{2}-\widehat{\mathbb{F}_{\beta}(\boldsymbol{U}) \boldsymbol{n}_{\mathrm{f}_{\beta}}}\right) .
\end{aligned}
$$

We conclude the proof by considering separately the case of the central and upwind numerical flux, and prove that $\mathcal{F}_{\mathrm{f}}\left(\boldsymbol{U}_{\mathrm{f}}^{+}, \boldsymbol{U}_{\mathrm{f}}^{-}\right)=-\mathcal{J}_{\mathrm{f}}$ for any face $\mathrm{f}$, where $\mathcal{J}_{\mathrm{f}}$ is defined in (82).

Central numerical scheme. The central numerical flux across face $f_{\beta}$ is formulated as:

$$
\widehat{\mathbb{F}_{\beta}(\boldsymbol{U}) \boldsymbol{n}_{\mathrm{f}_{\beta}}}=\mathbb{F}_{\beta}\left(\frac{\boldsymbol{U}_{\mathrm{f}_{\beta}}^{+}+\boldsymbol{U}_{\mathrm{f}_{\beta}}^{-}}{2}\right),
$$

and a direct substitution immediately yields that $\mathcal{F}_{\mathrm{f}_{\beta}}\left(\boldsymbol{U}_{\mathrm{f}_{\beta}}^{+}, \boldsymbol{U}_{\mathrm{f}_{\beta}}^{-}\right)=0$.

Upwind numerical scheme. The upwind numerical flux across face $f_{\beta}$ is formulated as:

$$
\widehat{\mathbb{F}_{\beta}(\boldsymbol{U}) \boldsymbol{n}_{\mathbf{f}_{\beta}}}=\mathbb{F}_{\beta}^{+}\left(\boldsymbol{U}_{\mathrm{f}_{\beta}}^{-}\right)+\mathbb{F}_{\beta}^{-}\left(\boldsymbol{U}_{\mathrm{f}_{\beta}}^{+}\right) .
$$

A direct substitution yields

$$
\begin{aligned}
\mathcal{F}_{\mathrm{f}_{\beta}}\left(\boldsymbol{U}_{\mathrm{f}_{\beta}}^{+}, \boldsymbol{U}_{\mathrm{f}_{\beta}}^{-}\right) & =-\left(\boldsymbol{U}_{\mathrm{f}_{\beta}}^{+}-\boldsymbol{U}_{\mathrm{f}_{\beta}}^{-}\right)^{T}\left(\mathbb{F}_{\beta} \frac{\left(\boldsymbol{U}_{\mathrm{f}_{\beta}}^{+}+\boldsymbol{U}_{\mathrm{f}_{\beta}}^{-}\right)}{2}-\mathbb{F}_{\beta}^{+} \boldsymbol{U}_{\mathrm{f}_{\beta}}^{-}-\mathbb{F}_{\beta}^{-} \boldsymbol{U}_{\mathrm{f}_{\beta}}^{+}\right) \\
& \left.=-\frac{1}{2}\left(\boldsymbol{U}_{\mathrm{f}_{\beta}}^{+}-\boldsymbol{U}_{\mathrm{f}_{\beta}}^{-}\right)^{T}\left(\left(\mathbb{F}_{\beta}^{+}+\mathbb{F}_{\beta}^{-}\right)\left(\boldsymbol{U}_{\mathrm{f}_{\beta}}^{+}+\boldsymbol{U}_{\mathrm{f}_{\beta}}^{-}\right)-2 \mathbb{F}_{\beta}^{+} \boldsymbol{U}_{\mathrm{f}_{\beta}}^{-}-2 \mathbb{F}_{\beta}^{-} \boldsymbol{U}_{\mathrm{f}_{\beta}}^{+}\right)\right) \\
& =-\frac{1}{2}\left(\boldsymbol{U}_{\mathrm{f}_{\beta}}^{+}-\boldsymbol{U}_{\mathrm{f}_{\beta}}^{-}\right)^{T}\left(\mathbb{F}_{\beta}^{+}-\mathbb{F}_{\beta}^{-}\right)\left(\boldsymbol{U}_{\mathrm{f}_{\beta}}^{+}-\boldsymbol{U}_{\mathrm{f}_{\beta}}^{-}\right) \\
& =-\frac{1}{2}\left(\boldsymbol{U}_{\mathrm{f}_{\beta}}^{+}-\boldsymbol{U}_{\mathrm{f}_{\beta}}^{-}\right)^{T}\left|\mathbb{F}_{\beta}\right|\left(\boldsymbol{U}_{\mathrm{f}_{\beta}}^{+}-\boldsymbol{U}_{\mathrm{f}_{\beta}}^{-}\right),
\end{aligned}
$$

which concludes the proof. 